\title{
Topological Field Theory of Time-Reversal Invariant Insulators
}

\author{
Xiao-Liang Qi, Taylor Hughes and Shou-Cheng Zhang \\ Department of Physics, Stanford University, Stanford, CA 94305
}

\begin{abstract}
We show that the fundamental time reversal invariant (TRI) insulator exists in $4+1$ dimensions, where the effective field theory is described by the $4+1$ dimensional Chern-Simons theory and the topological properties of the electronic structure is classified by the second Chern number. These topological properties are the natural generalizations of the time reversal breaking (TRB) quantum Hall insulator in $2+1$ dimensions. The TRI quantum spin Hall insulator in $2+1$ dimensions and the topological insulator in $3+1$ dimension can be obtained as descendants from the fundamental TRI insulator in $4+1$ dimensions through a dimensional reduction procedure. The effective topological field theory, and the $Z_{2}$ topological classification for the TRI insulators in $2+1$ and $3+1$ dimensions are naturally obtained from this procedure. All physically measurable topological response functions of the TRI insulators are completely described by the effective topological field theory. Our effective topological field theory predicts a number of novel and measurable phenomena, the most striking of which is the topological magneto-electric effect, where an electric field generates a magnetic field in the same direction, with an universal constant of proportionality quantized in odd multiples of the fine structure constant $\alpha=e^{2} / \hbar c$. Finally, we present a general classification of all topological insulators in various dimensions, and describe them in terms of a unified topological Chern-Simons field theory in phase space.
\end{abstract}

Contents

\section{Introduction}

II. TRB topological insulators in $2+1$ dimensions and its dimensional reduction 3

A. The first Chern number and topological response function in $(2+1)$ - $d$

B. Example: two band models

C. Dimensional reduction

D. $Z_{2}$ classification of particle-hole symmetric insulators in $(1+1)$-d

E. $Z_{2}$ classification of $(0+1)$-d particle-hole symmetric insulators

\section{Second Chern number and its physical} consequences

A. Second Chern number in $(4+1)$-d non-linear response

B. TRI topological insulators based on lattice Dirac models

IV. Dimensional reduction to $(3+1)$-d TRI insulators

A. Effective action of $(3+1)$-d insulators

B. Physical Consequences of the Effective Action $S_{3 D}$

C. $Z_{2}$ topological classification of time-reversal invariant insulators

D. Physical properties of $Z_{2}$-nontrivial insulators
VI. Unified theory of topological insulators

A. Phase space Chern-Simons theories

B. $Z_{2}$ topological insulator in generic dimensions 38
41

A. Conventions

B. Derivation of Eq. (54)

1. Topological invariance of Eq. (53)

2. Adiabatic deformation of arbitrary $h(\mathbf{k})$ to $h_{0}(\mathbf{k})$

3. Calculation of correlation function (53) for $h_{0}(\mathbf{k})$

C. The winding number in the non-linear response of Dirac-type models

D. Stability of edge theories in generic dimensions

References
V. Dimensional reduction to $(2+1)-\mathbf{d}$

A. Effective action of $(2+1)$-d insulators

B. $Z_{2}$ topological classification of TRI insulators 30

C. Physical properties of the $Z_{2}$ nontrivial insulators

\section{INTRODUCTION}

Most states or phases of condensed matter can be described by local order parameters and the associated broken symmetries. However, the quantum Hall (QH) state $1,2,3,4$ gives the first example of topological states of matter which have topological quantum numbers different from ordinary states of matter, and are described in the low energy limit by topological field theories. Soon after the discovery of the integer $\mathrm{QH}$ effect, the quantization of Hall conductance in units of $e^{2} / h$ was shown to be a general property of two-dimensional time reversal breaking (TRB) band insulators ${ }^{5}$. The integral of the curvature of the Berry's phase gauge field defined over the 
magnetic Brillouin zone (BZ) was shown to be a topological invariant called the first Chern number, which is physically measured as the quanta of the Hall conductance. In the presence of many-body interactions and disorder, the Berry curvature and the first Chern number can be defined over the space of twisted boundary conditions ${ }^{6}$. In the long wave length limit, both the integer and the fractional QH effect can be described by the topological Chern-Simons field theory $\underline{\underline{7}}$ in $2+1$ dimensions. This effective topological field theory captures all physically measurable topological effects, including the quantization of the Hall conductance, the fractional charge, and the statistics of quasi-particles $\stackrel{8}{\text {. }}$.

Insulators in $1+1$ dimensions can also have unique topological effects. Solitons in charge density wave insulators can have fractional charge or spin-charge separation ${ }^{9}$. The electric polarization of these insulators can be expressed in terms of the integral of the Berry's phase gauge field in momentum space $e^{10,11}$. During an adiabatic pumping cycle, the change of electric polarization, or the net charge pumped across the $1 \mathrm{D}$ insulator, is given by the integral of the Berry curvature over the hybrid space of momentum and the adiabatic pumping parameter. This integral is quantized to be a topological integer $\frac{12}{2}$. Both the charge of the soliton and the adiabatic pumping current can be obtained from the Goldstone-Wilczek formula 13 .

In this paper we shall show that the topological effects in the $1+1$ dimensional insulator can be obtained from the $\mathrm{QH}$ effect of the $2+1$ dimensional TRB insulator by a procedure called dimensional reduction. In this procedure one of the momenta is replaced by an adiabatic parameter, or field, and the Goldstone-Wilczek formula, and thus, all topological effects of the $1+1$ dimensional insulators, can be derived from the $2+1$ dimensional $\mathrm{QH}$ effect. The procedure of dimensional reduction can be generalized to the higher dimensional TRI insulators and beyond, which is the key result of this paper.

In recent years, the $\mathrm{QH}$ effect of the $2+1$ dimensional TRB insulators has been generalized to TRI insulators in various dimensions. The first example of a topologically non-trivial TRI state in condensed matter context was the $4 \mathrm{D}$ generalization of the $\mathrm{QH}$ effect (4DQH) proposed in Ref. 14. The effective theory of this model is given by the Chern-Simons topological field theory in $4+1$ dimensions $\frac{15}{}$. The quantum spin Hall (QSH) effect has been proposed in $2+1$ dimensional TRI quantum models 16,17 . The QSH insulator state has a gap for all bulk excitations, but has topologically protected gapless edge states, where opposite spin states counterpropagate $\frac{16,18,19}{}$. Recently the QSH state has been theoretically predicted 20 and experimentally observed in $\mathrm{HgTe}$ quantum wells 21 . TRI topological insulators have also been classified in $3+1$ dimensions ${ }^{22,23,24}$. These $3 \mathrm{D}$ states all carry spin Hall current in the insulating state ${ }^{25}$.

The topological properties of the $4+1$ dimensional TRI insulator can be described by the second Chern number defined over four dimensional momentum space. On the other hand, TRI insulators in $2+1$ and $3+1$ dimensions are described by a $Z_{2}$ topological invariant defined over momentum space $16,22,23,24,26,27,28,29,30$. In the presence of interactions and disorder, the momentum space $Z_{2}$ invariant is not well defined, however, one can define a more general $Z_{2}$ topological invariant in terms of spincharge separation associated with a $\pi$ flux ${ }^{31,32}$. One open question in this field concerns the relationship between the classification of the $4+1$ dimensional TRI insulator by the second Chern number and the classification of the $3+1$ and $2+1$ dimensional TRI insulators by the $Z_{2}$ number.

The effective theory of the $4+1$ dimensional TRI insulator is given by the topological Chern-Simons field theory 15,33 . While the $2+1$ dimensional Chern-Simons theory describes a linear topological response to an external $U(1)$ gauge field ${ }^{7,8}$, the $4+1$ dimensional ChernSimons theory describes a nonlinear topological response to an external $U(1)$ gauge field. The key outstanding theoretical problem in this field is the search for the topological field theory describing the TRI insulators in $2+1$ and $3+1$ dimensions, from which all measurable topological effects can be derived.

In this paper, we solve this outstanding problem by constructing topological field theories for the $2+1$ and $3+1$ dimensional TRI insulators using the procedure of dimensional reduction. We show that the $4+1$ dimensional topological insulator is the fundamental state from which all lower dimensional TRI insulators can be derived. This procedure is analogous to the dimensional reduction from the $2+1$ dimensional TRB topological insulator to the $1+1$ dimensional insulators. There is a deep reason why the fundamental TRB topological insulator exists in $2+1$ dimensions, while the fundamental TRI topological insulator exists in $4+1$ dimensions. The reason goes back to the Wigner-von Neumann classification ${ }^{34}$ of level crossings in TRB unitary quantum systems and the TRI symplectic quantum systems. Generically three parameters need to be tuned to obtain a level crossing in a TRB unitary system, while five parameters need to be tuned to obtain a level crossing in a TRI symplectic system. These level crossing singularities give rise to the non-trivial topological curvatures on the 2D and 4D parameter surfaces which enclose the singularities. Fundamental topological insulators are obtained in space dimensions where all these parameters are momentum variables. Once the fundamental TRI topological insulator is identified in $4+1$ dimensions, the lower dimensional versions of TRI topological insulators can be easily obtained by dimensional reduction. In this procedure, one or two momentum variables of the $4+1$ dimensional topological insulator are replaced by adiabatic parameters or fields, and the $4+1$ dimensional ChernSimons topological field theory is reduced to topological field theories involving both the external $U(1)$ gauge field and the adiabatic fields. For the $3+1$ TRI insulators, the topological field theory is given by that of the "axion Lagrangian", or the $3+1$ dimensional $\theta$ vacuum 
term, familiar in the context of quantum chromodynamics (QCD), where the adiabatic field plays the role of the axion field or the $\theta$ angle. From these topological field theories, all physically measurable topological effects of the $3+1$ and the $2+1$ dimensional TRI insulators can be derived. We predict a number of novel topological effects in this paper, the most striking of which is the topological magneto-electric (TME) effect, where an electric field induces a magnetic field in the same direction, with a universal constant of proportionality quantized in odd multiples of the fine structure constant $\alpha=e^{2} / \hbar c$. We also present an experimental proposal to measure this novel effect in terms of Faraday rotation. Our dimensional reduction procedure also naturally produces the $Z_{2}$ classification of the $3+1$ and the $2+1$ dimensional TRI topological insulators in terms of the integer second Chern class of the $4+1$ dimensional TRI topological insulators.

The remaining parts of the paper are organized as follows. In Sec. II we review the physical consequences of the first Chern number, namely the $(2+1)$-d QH effect and $(1+1)$-d fractional charge and topological pumping effects. We begin with the $(2+1)$-d time reversal breaking insulators and study the topological transport properties. We then present a dimensional reduction procedure that allows us to consider related topological phenomena in $(1+1)$-d and $(0+1)$-d. Subsequently, we define a $Z_{2}$ classification of these lower dimensional descendants which relies on the presence of a discrete particle-hole symmetry. This will serve as a review and a warm-up exercise for the more complicated phenomena we consider in the later sections. In Secs. III, IV, and V we discuss consequences of a non-trivial second Chern number beginning with a parent $(4+1)$-d topological insulator in Sec. III. In Secs. IV and V we continue studying the consequences of the second Chern number but in the physically realistic $(3+1)$-d and $(2+1)$-d models which are the descendants of the initial $(4+1)$-d system. We present effective actions describing all of the physical systems and their responses to applied electromagnetic fields. This provides the first effective field theory for the TRI topological insulators in $(3+1)$-d and $(2+1)$-d. For these two descendants of the $(4+1)$-d theory, we show that the $Z_{2}$ classification of the decedents are obtained from the 2 nd Chern number classification of the parent TRI insulator. Finally, in Sec. VI we unify all of the results into families of topological effective actions defined in a phase space formalism. From this we construct a family tree of all topological insulators, some of which are only defined in higher dimensions, and with topological $Z_{2}$ classifications which repeat every 8 dimensions.

This paper contains many new results on topological insulators, but it can also be read by advanced students as a pedagogical and self-contained introduction of topology applied to condensed matter physics. Physical models are presented in the familiar tight-binding forms, and all topological results can be derived by exact and explicit calculations, using techniques such as response theory al- ready familiar in condensed matter physics. During the course of reading this paper, we suggest the readers to consult Appendix A which covers all of our conventions.

\section{TRB TOPOLOGICAL INSULATORS IN $2+1$ DIMENSIONS AND ITS DIMENSIONAL REDUCTION}

In this section, we review the physics of the TRB topological insulators in $2+1$ dimensions. We shall use the example of a translationally invariant tight-binding mode ${ }^{35}$ which realizes the $\mathrm{QH}$ effect without Landau levels. We discuss the procedure of dimensional reduction, from which all topological effects of the $1+1$ dimensional insulators can be obtained. This section serves as a simple pedagogical example for the more complex case of the TRI insulators presented in Sec. III and IV.

\section{A. The first Chern number and topological response function in $(2+1)$-d}

In general, the tight-binding Hamiltonian of a $(2+1)-d$ band insulator can be expressed as

$$
H=\sum_{m, n ; \alpha, \beta} c_{m \alpha}^{\dagger} h_{m n}^{\alpha \beta} c_{n \beta}
$$

with $m, n$ the lattice sites and $\alpha, \beta=1,2, . . N$ the band indices for a $N$-band system. With translation symmetry $h_{m n}^{\alpha \beta}=h^{\alpha \beta}\left(\vec{r}_{m}-\vec{r}_{n}\right)$, the Hamiltonian can be diagonalized in a Bloch wavefunction basis:

$$
H=\sum_{\mathbf{k}} c_{\mathbf{k} \alpha}^{\dagger} h^{\alpha \beta}(\mathbf{k}) c_{\mathbf{k} \beta}
$$

The minimal coupling to an external electro-magnetic field is given by $h_{m n}^{\alpha \beta} \rightarrow h_{m n}^{\alpha \beta} e^{i A_{m n}}$ where $A_{m n}$ is a gauge potential defined on a lattice link with sites $m, n$ at the end. To linear order, the Hamiltonian coupled to the electro-magnetic field is obtained as

$$
H \simeq \sum_{\mathbf{k}} c_{\mathbf{k}}^{\dagger} h(\mathbf{k}) c_{\mathbf{k}}+\sum_{\mathbf{k}, \mathbf{q}} A^{i}(-\mathbf{q}) c_{\mathbf{k}+\mathbf{q} / \mathbf{2}}^{\dagger} \frac{\partial h(\mathbf{k})}{\partial k_{i}} c_{\mathbf{k}-\mathbf{q} / \mathbf{2}}
$$

with the band indices omitted. The DC response of the system to external field $A^{i}(\mathbf{q})$ can be obtained by the standard Kubo formula:

$$
\begin{aligned}
\sigma_{i j}= & \lim _{\omega \rightarrow 0} \frac{i}{\omega} Q_{i j}(\omega+i \delta), \\
Q_{i j}\left(i \nu_{m}\right)= & \frac{1}{\Omega \beta} \sum_{\mathbf{k}, n} \operatorname{tr}\left(J_{i}(\mathbf{k}) G\left(\mathbf{k}, i\left(\omega_{n}+\nu_{m}\right)\right)\right. \\
& \left.\cdot J_{j}(\mathbf{k}) G\left(\mathbf{k}, i \omega_{n}\right)\right),
\end{aligned}
$$

with the DC current $J_{i}(\mathbf{k})=\partial h(\mathbf{k}) / \partial k_{i}, i, j=x, y$, Green's function $G\left(\mathbf{k}, i \omega_{n}\right)=\left[i \omega_{n}-h(\mathbf{k})\right]^{-1}$, and $\Omega$ the area of the system. When the system is a band insulator 
with $M$ fully-occupied bands, the longitudinal conductance vanishes, i.e. $\sigma_{x x}=0$, as expected, while $\sigma_{x y}$ has the form shown in Ref. 5:

$$
\begin{aligned}
\sigma_{x y} & =\frac{e^{2}}{h} \frac{1}{2 \pi} \int d k_{x} \int d k_{y} f_{x y}(\mathbf{k}) \\
\text { with } f_{x y}(\mathbf{k}) & =\frac{\partial a_{y}(\mathbf{k})}{\partial k_{x}}-\frac{\partial a_{x}(\mathbf{k})}{\partial k_{y}} \\
a_{i}(\mathbf{k}) & =-i \sum_{\alpha \in \text { occ }}\left\langle\alpha \mathbf{k}\left|\frac{\partial}{\partial k_{i}}\right| \alpha \mathbf{k}\right\rangle, i=x, y .
\end{aligned}
$$

Physically, $a_{i}(\mathbf{k})$ is the $U(1)$ component of the Berry's phase gauge field (adiabatic connection) in momentum space. The quantization of the first Chern number

$$
C_{1}=\frac{1}{2 \pi} \int d k_{x} \int d k_{y} f_{x y}(\mathbf{k}) \in \mathbb{Z}
$$

is satisfied for any continuous states $|\alpha \mathbf{k}\rangle$ defined on the BZ.

Due to charge conservation, the $\mathrm{QH}$ response $j_{i}=$ $\sigma_{H} \epsilon^{i j} E_{j}$ also induces another response equation:

$$
\begin{aligned}
j_{i} & =\sigma_{H} \epsilon^{i j} E_{j} \\
& \Rightarrow \frac{\partial \rho}{\partial t}=-\nabla \cdot \mathbf{j}=-\sigma_{H} \nabla \times \mathbf{E}=\sigma_{H} \frac{\partial B}{\partial t} \\
& \Rightarrow \rho(B)-\rho_{0}=\sigma_{H} B
\end{aligned}
$$

where $\rho_{0}=\rho(B=0)$ is the charge density in the ground state. Equations (6) and (7) can be combined together in a covariant way:

$$
j^{\mu}=\frac{C_{1}}{2 \pi} \epsilon^{\mu \nu \tau} \partial_{\nu} A_{\tau}
$$

where $\mu, \nu, \tau=0,1,2$ are temporal and spatial indices. Here and below we will take the units $e=\hbar=1$ so that $e^{2} / h=1 / 2 \pi$.

The response equations (8) can be described by the topological Chern-Simons field theory of the external field $A_{\mu}$ :

$$
S_{\mathrm{eff}}=\frac{C_{1}}{4 \pi} \int d^{2} x \int d t A_{\mu} \epsilon^{\mu \nu \tau} \partial_{\nu} A_{\tau},
$$

in the sense that $\delta S_{\text {eff }} / \delta A_{\mu}=j^{\mu}$ recovers the response equations (8). Such an effective action is topologically invariant, in agreement with the topological nature of the first Chern number. All topological responses of the $\mathrm{QH}$ state are contained in the Chern-Simons theory ${ }^{8}$.

\section{B. Example: two band models}

To make the physical picture clearer, the simplest case of a two band model can be studied as an example $e^{35}$. The Hamiltonian of a two-band model can be generally written as

$$
h(\mathbf{k})=\sum_{a=1}^{3} d_{a}(\mathbf{k}) \sigma^{a}+\epsilon(\mathbf{k}) \mathbb{I}
$$

where $\mathbb{I}$ is the $2 \times 2$ identity matrix and $\sigma^{a}$ are the three Pauli matrices. Here we assume that the $\sigma^{a}$ represent a spin or pseudo-spin degree of freedom. If it is a real spin then the $\sigma^{a}$ are thus odd under time reversal. If If the $d_{a}(\mathbf{k})$ are odd in $\mathbf{k}$ then the Hamiltonian is time-reversal invariant. However, if any of the $d_{a}$ contain a constant term then the model has explicit time-reversal symmetry breaking. If the $\sigma^{a}$ are a pseudo-spin then one has to be more careful. Since, in this case, $\mathcal{T}^{2}=1$ then only $\sigma^{y}$ is odd under time-reversal (because it is imaginary) while $\sigma^{x}, \sigma^{z}$ are even. The identity matrix is even under time-reversal and $\epsilon(\mathbf{k})$ must be even in $\mathbf{k}$ to preserve time-reversal. The energy spectrum is easily obtained: $E_{ \pm}(\mathbf{k})=\epsilon(\mathbf{k}) \pm \sqrt{\sum_{a} d_{a}^{2}(\mathbf{k})}$. When $\sum_{a} d_{a}^{2}(\mathbf{k})>0$ for all $\mathbf{k}$ in the BZ, the two bands never touch each other. If we also require that $\max _{\mathbf{k}}\left(E_{-}(\mathbf{k})\right)<\min _{\mathbf{k}}\left(E_{+}(\mathbf{k})\right)$, so that the gap is not closed indirectly, then a gap always exists between the two bands of the system. In the single particle Hamiltonian $h(\mathbf{k})$, the vector $\mathbf{d}(\mathbf{k})$ acts as a "Zeeman field" applied to a "pseudospin" $\sigma_{i}$ of a two level system. The occupied band satisfies $(\mathbf{d}(\mathbf{k}) \cdot \sigma)|-, \mathbf{k}\rangle=-|\mathbf{d}(\mathbf{k})||-, \mathbf{k}\rangle$, which thus corresponds to the spinor with spin polarization in the $-\mathbf{d}(\mathbf{k})$ direction. Thus the Berry's phase gained by $|-, \mathbf{k}\rangle$ during an adiabatic evolution along some path $C$ in $\mathbf{k}$-space is equal to the Berry's phase a spin- $1 / 2$ particle gains during the adiabatic rotation of the magnetic field along the path $\mathbf{d}(C)$. This is known to be half of the solid angle subtended by $\mathbf{d}(C)$, as shown in Fig 1 . Consequently, the first Chern number $C_{1}$ is determined by the winding number of $\mathbf{d}(\mathbf{k})$ around the origin ${ }^{35.36}$ :

$$
C_{1}=\frac{1}{4 \pi} \int d k_{x} \int d k_{y} \hat{\mathbf{d}} \cdot \frac{\partial \hat{\mathbf{d}}}{\partial k_{x}} \times \frac{\partial \hat{\mathbf{d}}}{\partial k_{y}} .
$$

From the response equations we know that a non-zero $C_{1}$ implies a quantized Hall response. The Hall effect can only occur in a system with time-reversal symmetry breaking so if $C_{1} \neq 0$ then time-reversal symmetry is broken. Historically, the first example of such a two-band model with a non-zero Chern number was a honeycomb lattice model with imaginary next-nearest-neighbor hopping proposed by Haldane 37 .

To be concrete, we shall study a particular two band model introduced in Ref $\frac{35}{5}$, which is given by

$$
\begin{aligned}
h(\mathbf{k})= & \left(\sin k_{x}\right) \sigma_{x}+\left(\sin k_{y}\right) \sigma_{y} \\
& +\left(m+\cos k_{x}+\cos k_{y}\right) \sigma_{z},
\end{aligned}
$$

This Hamiltonian corresponds to the form (10) with $\epsilon(\mathbf{k}) \equiv 0$ and $d(\mathbf{k})=\left(\sin k_{x}, \sin k_{y}, m+\cos k_{x}+\cos k_{y}\right)$. The Chern number of this system is 35

$$
C_{1}=\left\{\begin{array}{cc}
1, & 0<m<2 \\
-1, & -2<m<0 \\
0, & \text { otherwise }
\end{array}\right.
$$

In the continuum limit, this model reduces to the $2+1$ dimensional massive Dirac Hamiltonian

$$
h(\mathbf{k})=k_{x} \sigma_{x}+k_{y} \sigma_{y}+(m+2) \sigma_{z}=\left(\begin{array}{cc}
m+2 & k_{x}-i k_{y} \\
k_{x}+i k_{y} & -m-2
\end{array}\right) .
$$




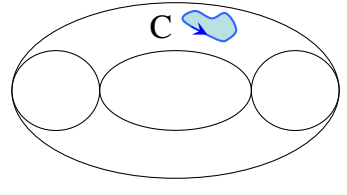

$B Z$ of $\mathbf{k}$
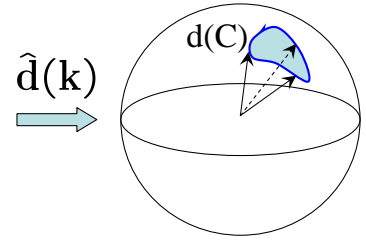

$\mathrm{S}_{2}$ of $\hat{\mathrm{d}}(\mathrm{k})$
G. 1: Illustration of the Berry's phase curvature in a twoband model. The Berry's phase $\oint_{C} \mathbf{A} \cdot \mathbf{d r}$ around a path $C$ in the BZ is half of the solid angle subtended by the image path $d(C)$ on the sphere $S_{2}$.

In a real space, this model can be expressed in tightbinding form as

$$
\begin{aligned}
H= & \sum_{n}\left[c_{n}^{\dagger} \frac{\sigma_{z}-i \sigma_{x}}{2} c_{n+\hat{x}}+c_{n}^{\dagger} \frac{\sigma_{z}-i \sigma_{y}}{2} c_{n+\hat{y}}+\text { h.c. }\right] \\
& +m \sum_{n} c_{n}^{\dagger} \sigma_{z} c_{n}
\end{aligned}
$$

Physically, such a model describes the quantum anomalous Hall effect realized with both strong spin-orbit coupling ( $\sigma_{x}$ and $\sigma_{y}$ terms) and ferromagnetic polarization $\left(\sigma_{z}\right.$ term). Initially this model was introduced for its simplicity in Ref. 35, however, recently, it was shown that it can be physically realized in $\mathrm{Hg}_{1-\mathrm{x}} \mathrm{Mn}_{\mathrm{x}} \mathrm{Te} / \mathrm{Cd}_{1-\mathrm{x}} \mathrm{Mn}_{\mathrm{x}} \mathrm{Te}$ quantum wells with a proper amount of $\mathrm{Mn}$ spin polarization ${ }^{38}$.

\section{Dimensional reduction}

To see how topological effects of $1+1$ dimensional insulators can be derived from the first Chern number and the $\mathrm{QH}$ effect through the procedure of dimensional reduction, we start by studying the $\mathrm{QH}$ system on a cylinder. An essential consequence of the nontrivial topology in the $\mathrm{QH}$ system is the existence of chiral edge states. For the simplest case with the first Chern number $C_{1}=1$, there is one branch of chiral fermions on each boundary. These edge states can be solved for explicitly by diagonalizing the Hamiltonian (14) in a cylindrical geometry. That is, with periodic boundary conditions in the $y$-direction and open boundary conditions in the $x$-direction, as shown in Fig2 (a). Note that with this choice $k_{y}$ is still a good quantum number. By defining the partial Fourier transformation

$$
c_{k_{y} \alpha}(x)=\frac{1}{\sqrt{L_{y}}} \sum_{y} c_{\alpha}(x, y) e^{i k_{y} y}
$$

(a)

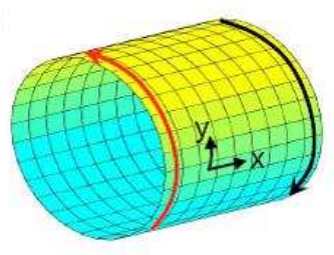

(b)

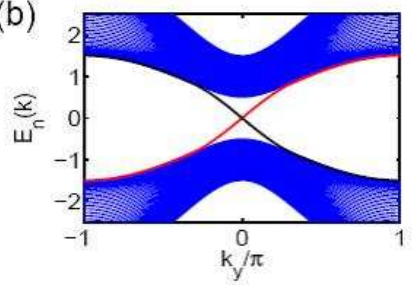

(c)

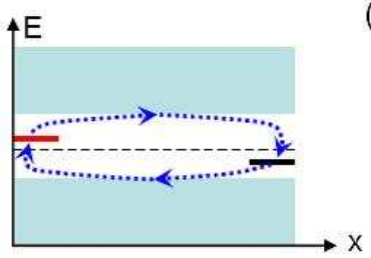

(d)

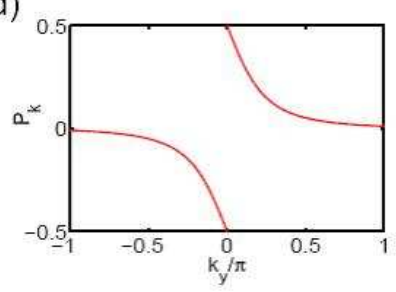

FIG. 2: (a) Illustration of a square lattice with cylindrical geometry and the chiral edge states on the boundary. The definition of $x$ and $y$ axis are also shown by black arrows. (b) One-d energy spectrum of the model in Eq. (12) with $m=-1.5$. The red and black line stands for the left and right moving edge states, respectively, while the blue lines are bulk energy levels. (c) Illustration of the edge states evolution for $k_{y}=0 \rightarrow 2 \pi$. The arrow shows the motion of end states in the space of center-of-mass position versus energy. (d) Polarization of the one-d system versus $k_{y}$. (See text)

with $(x, y)$ the coordinates of square lattice sites, the Hamiltonian can be rewritten as

$$
\begin{aligned}
H= & \sum_{k_{y}, x}\left[c_{k_{y}}^{\dagger}(x) \frac{\sigma_{z}-i \sigma_{x}}{2} c_{k_{y}}(x+1)+h . c .\right] \\
& +\sum_{k_{y}, x} c_{k_{y}}^{\dagger}(x)\left[\sin k_{y} \sigma_{y}+\left(m+\cos k_{y}\right) \sigma_{z}\right] c_{k_{y}}(x) \\
\equiv & \sum_{k_{y}} H_{1 \mathrm{D}}\left(k_{y}\right) .
\end{aligned}
$$

In this way, the $2 \mathrm{D}$ system can be treated as $L_{y}$ independent $1 \mathrm{D}$ tight-binding chains, where $L_{y}$ is the period of the lattice in the $y$-direction. The eigenvalues of the 1D Hamiltonian $H_{1 \mathrm{D}}\left(k_{y}\right)$ can be obtained numerically for each $k_{y}$, as shown in Fig. 2 (b). An important property of the spectrum is the presence of edge states, which lie in the bulk energy gap, and are spatially localized at the two boundaries: $x=0, L_{x}$. The chiral nature of the edge states can be seen from their energy spectrum. From Fig. 22 (b) we can see that the velocity $v=\partial E / \partial k$ is always positive for the left edge state and negative for the right one. The QH effect can be easily understood in this edge state picture by Laughlin's gauge argument ${ }^{3}$. Consider a constant electric field $E_{y}$ in the $y$-direction, which can be chosen as

$$
A_{y}=-E_{y} t, A_{x}=0 .
$$


The Hamiltonian is written $H=\sum_{k_{y}} H_{1 \mathrm{D}}\left(k_{y}+A_{y}\right)$ and the current along the $x$-direction is given by

$$
J_{x}=\sum_{k_{y}} J_{x}\left(k_{y}\right)
$$

with $J_{x}\left(k_{y}\right)$ the current of the $1 \mathrm{D}$ system. In this way, the Hall response of the 2D system is determined by the current response of the parameterized $1 \mathrm{D}$ systems $H_{1 \mathrm{D}}(q(t))$ to the temporal change of the parameter $q(t)=k_{y}+A_{y}(t)$. The gauge vector $A_{y}$ corresponds to a flux $\Phi=A_{y} L_{y}$ threading the cylinder. During a time period $0 \leq t \leq 2 \pi / L_{y} E_{y}$, the flux changes from 0 to $2 \pi$. The charge that flows through the system during this time is given by

$$
\begin{aligned}
\Delta Q & =\int_{0}^{\Delta t} d t \sum_{k_{y}} J_{x}\left(k_{y}\right) \\
& \left.\equiv \sum_{k_{y}} \Delta P_{x}\left(k_{y}\right)\right|_{0} ^{\Delta t}
\end{aligned}
$$

with $\Delta t=2 \pi / L_{y} E_{y}$. In the second equality we use the relation between the current and charge polarization $P_{x}\left(k_{y}\right)$ of the $1 \mathrm{D}$ systems $J_{x}\left(k_{y}\right)=d P_{x}\left(k_{y}\right) / d t$. In the adiabatic limit, the 1D system stays in the ground state of $H_{1 \mathrm{D}}(q(t))$, so that the change of polarization $\Delta P_{x}\left(k_{y}\right)$ is given by $\Delta P_{x}\left(k_{y}\right)=P_{x}\left(k_{y}-2 \pi / L_{y}\right)-P_{x}\left(k_{y}\right)$. Thus in the $L_{y} \rightarrow \infty$ limit $\Delta Q$ can be written as

$$
\Delta Q=-\oint_{0}^{2 \pi} d k_{y} \frac{\partial P_{x}\left(k_{y}\right)}{\partial k_{y}} .
$$

Therefore, the charge flow due to the Hall current generated by the flux through the cylinder equals the charge flow through the 1-dimensional system $H_{1 \mathrm{D}}\left(k_{y}\right)$, when $k_{y}$ is cycled adiabatically from 0 to $2 \pi$. From the QH response we know $\Delta Q=\sigma_{H} \Delta t E_{y} L_{y}=2 \pi \sigma_{H}$ is quantized as an integer, which is easy to understand in the $1 \mathrm{D}$ picture. During the adiabatic change of $k_{y}$ from 0 to $2 \pi$, the energy and position of the edge states will change, as shown in Fig 2 (c). Since the edge state energy is always increasing(decreasing) with $k_{y}$ for a state on the left (right) boundary, the charge is always "pumped" to the left for the half-filled system, which leads to $\Delta Q=-1$ for each cycle. This quantization can also be explicitly shown by calculating the polarization $P_{x}\left(k_{y}\right)$, as shown in Fig 2 (d), where the jump of $P_{x}$ by one leads to $\Delta Q=-1$. In summary, we have shown that the $\mathrm{QH}$ effect in the tight-binding model of Eq. (12) can be mapped to an adiabatic pumping effect $\frac{12}{2}$ by diagonalizing the system in one direction and mapping the momentum $k$ to a parameter.

Such a dimensional reduction procedure is not restricted to specific models, and can be generalized to any 2D insulators. For any insulator with Hamiltonian (2), we can define the corresponding $1 \mathrm{D}$ systems

$$
H_{1 \mathrm{D}}(\theta)=\sum_{k_{x}} c_{k_{x} \theta}^{\dagger} h\left(k_{x}, \theta\right) c_{k_{x} \theta}
$$

in which $\theta$ replaces the $y$-direction momentum $k_{y}$ and effectively takes the place of $q(t)$. When $\theta$ is timedependent, the current response can be obtained by a similar Kubo formula to Eq. (3), except that the summation over all $\left(k_{x}, k_{y}\right)$ is replaced by that over only $k_{x}$. More explicitly, such a linear response is defined as

$$
\begin{aligned}
J_{x}(\theta)= & G(\theta) \frac{d \theta}{d t} \\
G(\theta)= & \lim _{\omega \rightarrow 0} \frac{i}{\omega} Q(\omega+i \delta ; \theta) \\
Q\left(i \omega_{n} ; \theta\right)= & -\sum_{k_{x}, i \nu_{m}} \operatorname{tr}\left(J_{x}\left(k_{x} ; \theta\right) G_{1 \mathrm{D}}\left(k_{x}, i\left(\nu_{m}+\omega_{n}\right) ; \theta\right)\right. \\
& \left.\cdot \frac{\partial h\left(k_{x} ; \theta\right)}{\partial \theta} G_{1 \mathrm{D}}\left(k_{x}, i \omega_{n} ; \theta\right)\right) \frac{1}{L_{x} \beta} .
\end{aligned}
$$

Similar to Eq. (4) of the 2D case, the response coefficient $G(k)$ can be expressed in terms of a Berry's phase gauge field as

$$
\begin{aligned}
G(\theta) & =-\oint \frac{d k_{x}}{2 \pi} f_{x \theta}\left(k_{x}, \theta\right) \\
& =\oint \frac{d k_{x}}{2 \pi}\left(\frac{\partial a_{x}}{\partial \theta}-\frac{\partial a_{\theta}}{\partial k_{x}}\right)
\end{aligned}
$$

with the sum rule

$$
\int G(\theta) d \theta=C_{1} \in \mathbb{Z}
$$

If we choose a proper gauge so that $a_{\theta}$ is always singlevalued, the expression of $G(\theta)$ can be further simplified to

$$
G(\theta)=\frac{\partial}{\partial \theta}\left(\oint \frac{d k_{x}}{2 \pi} a_{x}\left(k_{x}, \theta\right)\right) \equiv \frac{\partial P(\theta)}{\partial \theta} .
$$

Physically, the loop integral

$$
P(\theta)=\oint d k_{x} a_{x} / 2 \pi
$$

is nothing but the charge polarization of the $1 \mathrm{D}$ system $^{10,11}$, and the response equation (20) simply becomes $J_{x}=\partial P / \partial t$. Since the polarization $P$ is defined as the shift of the electron center-of-mass position away from the lattice sites, it is only well-defined modulo 1 . Consequently, the change $\Delta P=P(\theta=2 \pi)-P(\theta=0)$ through a period of adiabatic evolution is an integer equal to $-C_{1}$, and corresponds to the charge pumped through the system. Such a relation between quantized pumping and the first Chern number was shown by Thouless 12 .

Similar to the QH case, the current response can lead to a charge density response, which can be determined by the charge conservation condition. When the parameter $\theta$ has a smooth spatial dependence $\theta=\theta(x, t)$, the response equation (20) still holds. From the continuity equation we obtain

$$
\begin{aligned}
\frac{\partial \rho}{\partial t} & =-\frac{\partial J_{x}}{\partial x}=-\frac{\partial^{2} P(\theta)}{\partial x \partial t} \\
\Rightarrow \rho & =-\frac{\partial P(\theta)}{\partial x}
\end{aligned}
$$


in which $\rho$ is defined with respect to the background charge. Similar to Eq. (8), the density and current response can be written together as

$$
j_{\mu}=-\epsilon_{\mu \nu} \frac{\partial P(\theta(x, t))}{\partial x_{\nu}}
$$

where $\mu, \nu=0,1$ are time and space. It should be noted that only differentiation with respect to $x, t$ appears in Eq. (26). This means, as expected, the current and density response of the system do not depend on the parametrization. In general, when the Hamiltonian has smooth space and time dependence, the single particle Hamiltonian $h(k)$ becomes $h(k, x, t) \equiv h(k, \theta(x, t))$, which has the eigenstates $|\alpha ; k, x, t\rangle$ with $\alpha$ the band index. Then relabelling $t, x, k$ as $q_{A}, A=0,1,2$ we can define the phase space Berry's phase gauge field

$$
\begin{aligned}
\mathcal{A}_{A} & =-i \sum_{\alpha}\left\langle\alpha ; q_{A}\left|\frac{\partial}{\partial q_{A}}\right| \alpha ; q_{A}\right\rangle \\
\mathcal{F}_{A B} & =\partial_{A} \mathcal{A}_{B}-\partial_{B} \mathcal{A}_{A}
\end{aligned}
$$

and the phase space current

$$
j_{A}^{P}=-\frac{1}{4 \pi} \epsilon_{A B C} \mathcal{F}_{B C} .
$$

The physical current is obtained by integration over the wavevector manifold:

$$
j_{\mu}=\int d k j_{\mu}^{P}=-\int \frac{d k}{2 \pi} \epsilon^{\mu 2 \nu} \mathcal{F}_{2 \nu}
$$

where $\mu, \nu=0,1$. This recovers Eq. (26). Note that we could have also looked at the component $j_{k}=\int d k j_{k}^{P}$ but this current does not have a physical interpretation.

Before moving to the next topic, we would like to apply this formalism to the case of the Dirac model, which reproduces the well-known result of fractional charge in the Su-Schrieffer-Heeger (SSH) mode $\mathbf{l}^{\underline{9}}$, or equivalently the Jackiw-Rebbi model ${ }^{39}$. To see this, consider the following slightly different version of the tight-binding model (12):

$$
\begin{aligned}
h(k, \theta)= & \sin k \sigma_{x}+(\cos k-1) \sigma_{z} \\
& +m\left(\sin \theta \sigma_{y}+\cos \theta \sigma_{z}\right)
\end{aligned}
$$

with $m>0$. In the limit $m \ll 1$, the Hamiltonian has the continuum limit $h(k, \theta) \simeq k \sigma_{x}+m\left(\sin \theta \sigma_{y}+\cos \theta \sigma_{z}\right)$, which is the continuum Dirac model in $(1+1)$-d, with a real mass $m \cos \theta$ and an imaginary mass $m \sin \theta$. As discussed in Sec. IIB, the polarization $\oint d k_{x} a_{x} / 2 \pi$ is determined by the solid angle subtended by the curve $\mathbf{d}(k)=(\sin k, m \sin \theta, m \cos \theta+\cos k-1)$, as shown in Fig. 3. In the limit $m \ll 1$ one can show that the solid angle $\Omega(\theta)=2 \theta$ so that $P(\theta) \simeq \theta / 2 \pi$, in which case Eq. (26) reproduces the Goldstone-Wilczek formula 13 :

$$
j_{\mu}=-\epsilon_{\mu \nu} \partial_{\nu} \theta
$$

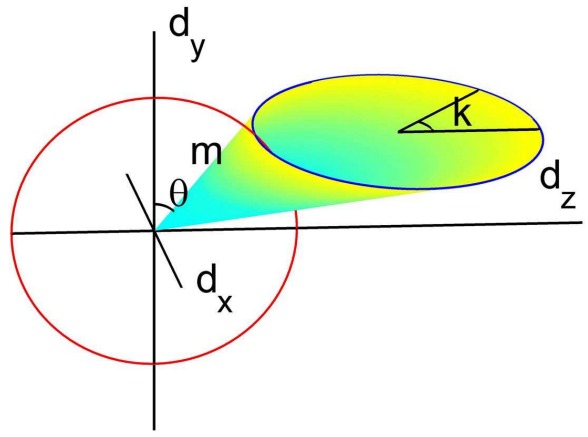

FIG. 3: Illustration of the $\mathbf{d}(k, \theta)$ vector for the $1 \mathrm{D}$ Dirac model (30). The horizontal blue circle shows the orbit of $\mathbf{d}(k)$ vector in the $3 \mathrm{D}$ space for $k \in[0,2 \pi)$ with $\theta$ fixed. The red circle shows the track of the blue circle under the variation of $\theta$. The cone shows the solid angle $\Omega(\theta)$ surrounded by the $\mathbf{d}(k)$ curve, which is $4 \pi$ times the polarization $P(\theta)$.

Specifically, a charge $Q=-\int_{-\infty}^{\infty}(d \theta / d x)(d x / 2 \pi)=$ $-(\theta(+\infty)-\theta(-\infty)) / 2 \pi$ is carried by a domain wall of the $\theta$ field. In particular, for an anti-phase domain wall, $\theta(+\infty)-\theta(-\infty)=\pi$, we obtain fractional charge $q=1 / 2$. Our phase space formula (28) is a new result, and it provides a generalization of the Goldstone-Wilczek formula to the most general one-dimensional insulator.

\section{D. $Z_{2}$ classification of particle-hole symmetric insulators in $(1+1)-d$}

In the last subsection, we have shown how the first Chern number of a Berry's phase gauge field appears in an adiabatic pumping effect and the domain wall charge of one-dimensional insulators. In these cases, an adiabatic spatial or temporal variation of the singleparticle Hamiltonian, through its parametric dependence on $\theta(x, t)$, is required to define the Chern number. In other words, the first Chern number is defined for $a p a$ rameterized family of Hamiltonians $h(k, x, t)$, rather than for a single 1D Hamiltonian $h(k)$. In this subsection, we will show a different application of the first Chern number, in which a $Z_{2}$ topological classification is obtained for particle-hole symmetric insulators in $1 D$. Such a relation between Chern number and $Z_{2}$ topology can be easily generalized to the more interesting case of second Chern number, where a similar $Z_{2}$ characterization is obtained for TRI insulators, as will be shown in Sec. IVC and $\mathrm{VB}$.

For a one-dimensional tight-binding Hamiltonian $H=$ $\sum_{m n} c_{m \alpha}^{\dagger} h_{m n}^{\alpha \beta}(k) c_{n \beta}$, the particle-hole transformation is defined by $c_{m \alpha} \rightarrow C^{\alpha \beta} c_{m \beta}^{\dagger}$, where the charge conjugation matrix $C$ satisfies $C^{\dagger} C=\mathbb{I}$ and $C^{*} C=\mathbb{I}$. Under periodic 
boundary conditions the symmetry requirement is

$$
\begin{aligned}
H & =\sum_{k} c_{k}^{\dagger} h(k) c_{k}=\sum_{k} c_{-k} C^{\dagger} h(k) C c_{-k}^{\dagger} \\
& \Rightarrow C^{\dagger} h(-k) C=-h^{T}(k) .
\end{aligned}
$$

From Eq. (32) it is straightforward to see the symmetry of the energy spectrum: if $E$ is an eigenvalue of $h(0)$, so is $-E$. Consequently, if the dimension of $h(k)$ is odd, there must be at least one zero mode with $E=0$. Since the chemical potential is constrained to vanish by the traceless condition of $h$, such a particle-hole symmetric system cannot be gapped unless the dimension of $h(k)$ is even. Since we are only interested in the classification of insulators, we will focus on the case with $2 N$ bands per lattice site.

Now consider two particle-hole symmetric insulators with Hamiltonians $h_{1}(k)$ and $h_{2}(k)$, respectively. In general, a continuous interpolation $h(k, \theta), \theta \in[0, \pi]$ between them can be defined so that

$$
h(k, 0)=h_{1}(k), h(k, \pi)=h_{2}(k)
$$

Moreover, it is always possible to find a proper parametrization so that $h(k, \theta)$ is gapped for all $\theta \in[0, \pi]$. In other words, the topological space of all 1D insulating Hamiltonians $h(k, \theta)$ is connected, which is a consequence of the Wigner-Von Neumann theorem ${ }^{34}$.

Suppose $h(k, \theta)$ is such a "gapped interpolation" between $h_{1}(k)$ and $h_{2}(k)$. In general, $h(k, \theta)$ for $\theta \in(0, \pi)$ doesn't necessarily satisfy the particle-hole symmetry. For $\theta \in[\pi, 2 \pi]$, define

$$
h(k, \theta)=-\left(C^{-1} h(-k, 2 \pi-\theta) C\right)^{T} .
$$

We choose this parameterization so that if we replaced $\theta$ by a momentum wavevector then the corresponding higher dimensional Hamiltonian would be particlehole symmetric. Due to the particle-hole symmetry of $h(k, \theta=0)$ and $h(k, \theta=\pi), h(k, \theta)$ is continuous for $\theta \in[0,2 \pi]$, and $h(k, 2 \pi)=h(k, 0)$. Consequently, the adiabatic evolution of $\theta$ from 0 to $2 \pi$ defines a cycle of adiabatic pumping in $h(k, \theta)$, and a first Chern number can be defined in the $(k, \theta)$ space. As discussed in Sec. IIC, the Chern number $C[h(k, \theta)]$ can be expressed as a winding number of the polarization

$$
\begin{aligned}
C[h(k, \theta)] & =\oint d \theta \frac{\partial P(\theta)}{\partial \theta} \\
P(\theta) & =\oint \frac{d k}{2 \pi} \sum_{E_{\alpha}(k)<0}(-i)\left\langle k, \theta ; \alpha\left|\partial_{k}\right| k, \theta ; \alpha\right\rangle
\end{aligned}
$$

where the summation is carried out over the occupied bands. In general, two different parameterizations $h(k, \theta)$ and $h^{\prime}(k, \theta)$ can lead to different Chern numbers $C[h(k, \theta)] \neq C\left[h^{\prime}(k, \theta)\right]$. However, the symmetry constraint in Eq. (34) guarantees that the two Chern numbers always differ by an even integer: $C[h(k, \theta)]-$ $C\left[h^{\prime}(k, \theta)\right]=2 n, n \in \mathbb{Z}$.
To prove this conclusion, we first study the behavior of $P(\theta)$ under a particle-hole transformation. For an eigenstate $|k, \theta ; \alpha\rangle$ of the Hamiltonian $h(k, \theta)$ with eigenvalue $E_{\alpha}(k, \theta)$, Eq. (34) leads to

$$
h(-k, 2 \pi-\theta) C|k, \theta ; \alpha\rangle^{*}=-E_{\alpha}(k) C|k, \theta ; \alpha\rangle^{*}
$$

in which $|k, \theta ; \alpha\rangle^{*}$ is the complex conjugate state: $|k, \theta ; \alpha\rangle^{*}=\sum_{m, \beta}(\langle m, \beta \mid k, \theta ; \alpha\rangle)^{*}|m, \beta\rangle$ where $m, \beta$ are the position space lattice, and orbital index respectively. Thus $C|k, \theta ; \alpha\rangle^{*} \equiv|-k, 2 \pi-\theta ; \bar{\alpha}\rangle$ is an eigenstate of $h(-k, 2 \pi-\theta)$ with energy $E_{\bar{\alpha}}(k, 2 \pi-\theta)=-E_{\alpha}(k, \theta)$ and momentum $-k$. Such a mapping between eigenstates of $h(k, \theta)$ and $h(-k, 2 \pi-\theta)$ is one-to-one. Thus

$$
\begin{aligned}
P(\theta)= & \oint \frac{d k}{2 \pi} \sum_{E_{\alpha}(k)<0}(-i)\left\langle k, \theta ; \alpha\left|\partial_{k}\right| k, \theta ; \alpha\right\rangle \\
= & \oint \frac{d k}{2 \pi} \sum_{E_{\bar{\alpha}}(-k)>0}(-i)(\langle-k, 2 \pi-\theta ; \bar{\alpha}|)^{*} \\
& \cdot \partial_{k}|-k, 2 \pi-\theta ; \bar{\alpha}\rangle^{*} \\
= & -P(2 \pi-\theta) .
\end{aligned}
$$

Since $P(\theta)$ is only well-defined modulo 1 , the equality (36) actually means $P(\theta)+P(2 \pi-\theta)=0 \bmod 1$. Consequently, for $\theta=0$ or $\pi$ we have $2 \pi-\theta=\theta \bmod 2 \pi$, so that $P(\theta)=0$ or $1 / 2$. In other words, the polarization $P$ is either 0 or $1 / 2$ for any particle-hole symmetric insulator, which thus defines a classification of particlehole symmetric insulators. If two systems have different $P$ value, they cannot be adiabatically connected without breaking the particle-hole symmetry, because $P(\bmod 1)$ is a continuous function during adiabatic deformation, and a $P$ value other than 0 and $1 / 2$ breaks particle-hole symmetry. Though such an argument explains physically why a $Z_{2}$ classification is defined for particle-hole symmetric system, it is not so rigorous. As discussed in the derivation from Eq. (21) to Eq. (23), the definition $P(\theta)=\oint d k a_{k} / 2 \pi$ relies on a proper gauge choice. To avoid any gauge dependence, a more rigorous definition of the $Z_{2}$ classification is shown below, which only involves the gauge invariant variable $\partial P(\theta) / \partial \theta$ and Chern number $C_{1}$.

To begin with, the symmetry (36) leads to

$$
\int_{0}^{\pi} d P(\theta)=\int_{\pi}^{2 \pi} d P(\theta)
$$

which is independent of gauge choice since only the change of $P(\theta)$ is involved. This equation shows that the change of polarization during the first half and the second half of the closed path $\theta \in[0,2 \pi]$ are always the same.

Now consider two different parameterizations $h(k, \theta)$ and $h^{\prime}(k, \theta)$, satisfying $h(k, 0)=h^{\prime}(k, 0)=h_{1}(k)$, $h(k, \pi)=h^{\prime}(k, \pi)=h_{2}(k)$. Denoting the polarization $P(\theta)$ and $P^{\prime}(\theta)$ corresponding to $h(k, \theta)$ and $h^{\prime}(k, \theta)$, respectively, the Chern number difference between $h$ and 
(a)

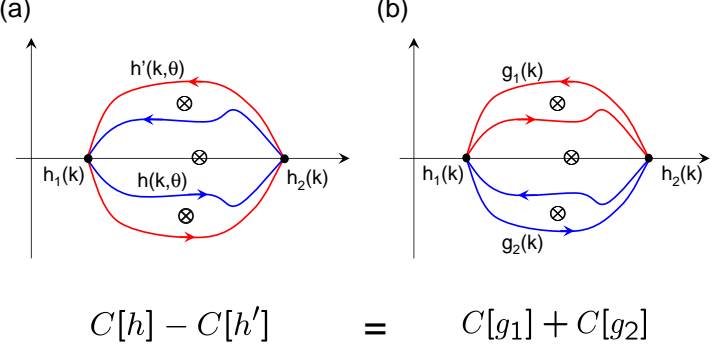

FIG. 4: Illustration of the interpolation between two particlehole symmetric Hamiltonians $h_{1}(k)$ and $h_{2}(k)$.

$h^{\prime}$ is given by

$$
C[h]-C\left[h^{\prime}\right]=\int_{0}^{2 \pi} d \theta\left(\frac{\partial P(\theta)}{\partial \theta}-\frac{\partial P^{\prime}(\theta)}{\partial \theta}\right) .
$$

Define the new interpolations $g_{1}(k, \theta)$ and $g_{2}(k, \theta)$ as

$$
\begin{aligned}
& g_{1}(k, \theta)=\left\{\begin{array}{cc}
h(k, \theta), & \theta \in[0, \pi] \\
h^{\prime}(k, 2 \pi-\theta), & \theta \in[\pi, 2 \pi]
\end{array}\right. \\
& g_{2}(k, \theta)=\left\{\begin{array}{cc}
h^{\prime}(k, 2 \pi-\theta), & \theta \in[0, \pi] \\
h(k, \theta), & \theta \in[\pi, 2 \pi]
\end{array}\right.
\end{aligned}
$$

$g_{1}(k, \theta)$ and $g_{2}(k, \theta)$ are obtained by recombination of the two paths $h(k, \theta)$ and $h^{\prime}(k, \theta)$, as shown in Fig. 4. From the construction of $g_{1}$ and $g_{2}$, it is straightforward to see that

$$
\begin{aligned}
& C\left[g_{1}\right]=\int_{0}^{\pi} d \theta\left(\frac{\partial P(\theta)}{\partial \theta}-\frac{\partial P^{\prime}(\theta)}{\partial \theta}\right) \\
& C\left[g_{2}\right]=\int_{\pi}^{2 \pi} d \theta\left(\frac{\partial P(\theta)}{\partial \theta}-\frac{\partial P^{\prime}(\theta)}{\partial \theta}\right) .
\end{aligned}
$$

Thus $C[h]-C\left[h^{\prime}\right]=C\left[g_{1}\right]+C\left[g_{2}\right]$. On the other hand, from Eq. (37) we know $C\left[g_{1}\right]=C\left[g_{2}\right]$, so that $C[h]-C\left[h^{\prime}\right]=2 C\left[g_{1}\right]$. Since $C\left[g_{1}\right] \in \mathbb{Z}$, we obtain that $C[h]-C\left[h^{\prime}\right]$ is even for any two interpolations $h(k, \theta)$ and $h^{\prime}(k, \theta)$ between $h_{1}(k)$ and $h_{2}(k)$. Intuitively, such a conclusion simply comes from the fact that the Chern number $C[h]$ and $C\left[h^{\prime}\right]$ can be different only if there are singularities between these two paths, while the positions of the singularities in the parameter space are always symmetric under particle-hole symmetry, as shown in Fig. 4.

Based on the discussions above, we can define the "relative Chern parity" as

$$
N_{1}\left[h_{1}(k), h_{2}(k)\right]=(-1)^{C[h(k, \theta)]},
$$

which is independent of the choice of interpolation $h(k, \theta)$, but only determined by the Hamiltonians $h_{1}(k), h_{2}(k)$. Moreover, for any three particle-hole symmetric Hamiltonians $h_{1}(k), h_{2}(k), h_{3}(k)$, it is easy to prove that the Chern parity satisfies the following associative law:

$$
N_{1}\left[h_{1}(k), h_{2}(k)\right] N_{1}\left[h_{2}(k), h_{3}(k)\right]=N_{1}\left[h_{1}(k), h_{3}(k)\right] .
$$

Consequently, $N_{1}\left[h_{1}(k), h_{2}(k)\right]=1$ defines an equivalence relation between any two particle-hole symmetric Hamiltonians, which thus classifies all the particle-hole symmetric insulators into two classes. To define these two classes more explicitly, one can define a "vacuum" Hamiltonian as $h_{0}(k) \equiv h_{0}$, where $h_{0}$ is an arbitrary matrix which does not depend on $k$ and which satisfies the particle-hole symmetry constraint $C^{\dagger} h_{0} C=-h_{0}^{T}$. Thus $h_{0}$ describes a totally local system, in which there is no hopping between different sites. Taking such a trivial system as a reference Hamiltonian, we can define $N_{1}\left[h_{0}(k), h(k)\right] \equiv N_{1}[h(k)]$ as a $Z_{2}$ topological quantum number of the Hamiltonian $h(k)$. All the Hamiltonians $h(k)$ with $N_{1}\left[h_{0}(k), h(k)\right]=1$ are classified as $Z_{2}$ trivial, while those with $N_{1}\left[h_{0}(k), h(k)\right]=-1$ are considered as $Z_{2}$ nontrivial. (Again, this classification doesn't depend on the choice of "vacuum" $h_{0}$, since any two vacua are equivalent.)

Despite its abstract form, such a topological characterization has a direct physical consequence. For a $Z_{2}$ nontrivial Hamiltonian $h_{1}(k)$, an interpolation $h(k, \theta)$ can be defined so that $h(k, 0)=h_{0}, h(k, \pi)=h_{1}(k)$, and the Chern number $C[h(k, \theta)]$ is an odd integer. If we study the one-dimensional system $h(k, \theta)$ with open boundary conditions, the tight binding Hamiltonian can be rewritten in real space as

$$
h_{m n}(\theta)=\frac{1}{\sqrt{L}} \sum_{k} e^{i k\left(x_{m}-x_{n}\right)} h(k, \theta), \forall 1 \leq m, n \leq L .
$$

As discussed in Sec. IIC there are mid-gap end states in the energy spectrum of $h_{m n}(\theta)$ as a consequence of the non-zero Chern number. When the Chern number $C[h(k, \theta)]=2 n-1, n \in \mathbb{Z}$, there are values $\theta_{s}^{L} \in[0,2 \pi), s=1,2, . .2 n-1$ for which the Hamiltonian $h_{m n}\left(\theta_{s}\right)$ has zero energy localized states on the left end of the $1 \mathrm{D}$ system, and the same number of $\theta_{s}^{R}$ values where zero energy states are localized on the right end, as shown in Fig. 5. Due to the particle-hole symmetry between $h_{m n}(\theta)$ and $h_{m n}(2 \pi-\theta)$, zero levels always appear in pairs at $\theta$ and $2 \pi-\theta$. Consequently, when the Chern number is odd, there must be a zero level at $\theta=0$ or $\theta=\pi$. Since $\theta=0$ corresponds to a trivial insulator with flat bands and no end states, the localized zero mode has to appear at $\theta=\pi$. In other words, one zero energy localized state (or an odd number of such states) is confined at each open boundary of a $Z_{2}$ nontrivial particle-hole symmetric insulator.

The existence of a zero level leads to an important physical consequence - a half charge on the boundary of the nontrivial insulator. In a periodic system when the chemical potential vanishes, the average electron density on each site is $\bar{n}_{m}=\left\langle\sum_{\alpha} c_{m \alpha}^{\dagger} c_{m \alpha}\right\rangle=N$ when there 
(a)

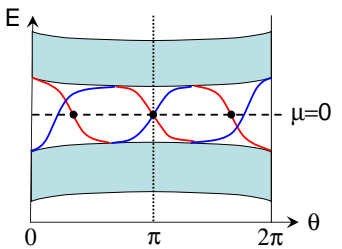

(b)

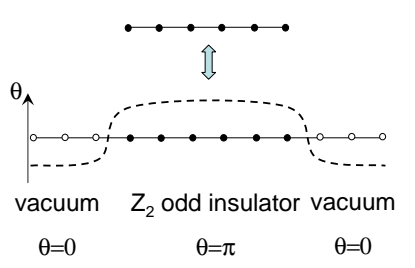

(c)

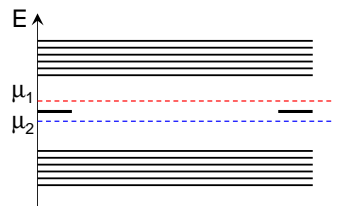

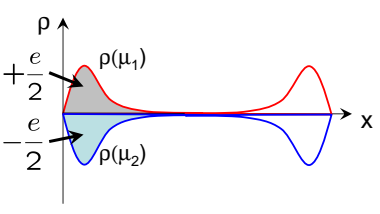

By using Eq.. (37) we obtain

$$
Q_{d}=\frac{e}{2} \int_{0}^{2 \pi} d P(\theta)=\frac{e}{2} C[h(k, \theta)] .
$$

It should be noted that an integer charge can always be added by changing the filling of local states, which means $Q_{d}$ is only fixed modulo $e$. Consequently, a $\pm e / 2$ charge is carried by the domain wall if and only if the Chern number is odd, i.e., when the insulator is nontrivial.

\section{E. $Z_{2}$ classification of $(0+1)$-d particle-hole symmetric insulators}

In the last subsection, we have shown how a $Z_{2}$ classification of $(1+1)$-d particle-hole symmetric insulators is defined by dimensional reduction from $(2+1)$-d systems. Such a dimensional reduction can be repeated once more to study $(0+1)$-d systems, that is, a singlesite problem. In this subsection we will show that a $Z_{2}$ classification of particle-hole symmetric Hamiltonians in $(0+1)$-d is also obtained by dimensional reduction. Although such a classification by itself is not as interesting as the higher dimensional counterparts, it does provide a simplest example of the "dimensional reduction chain" $(2+1)-\mathrm{d} \rightarrow(1+1)-\mathrm{d} \rightarrow(0+1)-\mathrm{d}$, which can be later generalized to its higher-dimensional counterpart $(4+1)$ $\mathrm{d} \rightarrow(3+1)-\mathrm{d} \rightarrow(2+1)-\mathrm{d}$. In other words, the $Z_{2}$ classification of the $(0+1)$-d particle-hole symmetric insulators can help us to understand the classification of $(2+1)$-d TRI insulators as it is dimensionally reduced from the $(4+1)$-d TRI insulator.

For a free, single-site fermion system with Hamiltonian matrix $h$, the particle-hole symmetry is defined as

$$
C^{\dagger} h C=-h^{T}
$$

Given any two particle-hole symmetric Hamiltonians $h_{1}$ and $h_{2}$, we follow the same procedure as the last subsection and define a continuous interpolation $h(\theta), \theta \in$ $[0,2 \pi]$ satisfying

$$
h(0)=h_{1}, h(\pi)=h_{2}, C^{\dagger} h(\theta) C=-h(2 \pi-\theta)^{T},
$$

where $h(\theta)$ is gapped for all $\theta$. The Hamiltonian $h(\theta)$ is the dimensional reduction of a $(1+1)$-d Hamiltonian $h(k)$, with the wavevector $k$ replaced by the parameter $\theta$. The constraint (46) is identical to the particle-hole symmetry condition (32), so that $h(\theta)$ corresponds to a particle-hole symmetric $(1+1)$-d insulator. As shown in last subsection, $h(\theta)$ is classified by the value of the "Chern parity" $N_{1}[h(\theta)]$. If $N_{1}[h(\theta)]=-1$, no continuous interpolation preserving particle-hole symmetry can be defined between $h(\theta)$ and the vacuum Hamiltonian $h(\theta)=h_{0}, \forall \theta \in$ $[0,2 \pi]$. To obtain the classification of $(0+1)$-d Hamiltonians, consider two different interpolations $h(\theta)$ and $h^{\prime}(\theta)$ between $h_{1}$ and $h_{2}$. According to the associative law (42), we know $N_{1}[h(\theta)] N_{1}\left[h^{\prime}(\theta)\right]=N_{1}\left[h(\theta), h^{\prime}(\theta)\right]$,

$$
Q_{d}=e \int_{-\infty}^{+\infty} d x \frac{\partial P(\theta(x))}{\partial x}=e \int_{0}^{\pi} d P(\theta)
$$


where $N_{1}\left[h(\theta), h^{\prime}(\theta)\right]$ is the relative Chern parity between two interpolations. In the following we will prove $N_{1}\left[h(\theta), h^{\prime}(\theta)\right]=1$ for any two interpolations $h$ and $h^{\prime}$ satisfying condition (46). As a result, $N_{1}[h(\theta)]$ is independent of the choice of interpolation between $h_{1}$ and $h_{2}$, so that $N_{0}\left[h_{1}, h_{2}\right] \equiv N_{1}[h(\theta)]$ can be defined as a function of $h_{1}$ and $h_{2}$. The $Z_{2}$ quantity $N_{0}$ defined for $(0+1)$-d Hamiltonians plays exactly the same role as $N_{1}\left[h(k), h^{\prime}(k)\right]$ in the $(1+1)$-d case, from which a $Z_{2}$ classification can be defined.

To prove $N_{1}\left[h(\theta), h^{\prime}(\theta)\right]=1$ for any two interpolations, first define a continuous deformation $g(\theta, \varphi)$ between $h(\theta)$ and $h^{\prime}(\theta)$, which satisfies the conditions below:

$$
\begin{aligned}
g(\theta, \varphi=0) & =h(\theta), g(\theta, \varphi=\pi)=h^{\prime}(\theta) \\
g(0, \varphi) & =h_{1}, g(\pi, \varphi)=h_{2} \\
C^{\dagger} g(\theta, \varphi) C & =-g(2 \pi-\theta, 2 \pi-\varphi)^{T}
\end{aligned}
$$

From the discussions in last subsection it is easy to confirm that such a continuous interpolation is always possible, in which $g(\theta, \varphi)$ is gapped for all $\theta$ and $\varphi$. In the two-dimensional parameter space $\theta, \varphi$ one can define the Berry phase gauge field and the first Chern number $C_{1}[g(\theta, \varphi)]$. By the definition of the Chern parity, we have $N_{1}\left[h(\theta), h^{\prime}(\theta)\right]=(-1)^{C_{1}[g(\theta, \varphi)]}$. However, the parameterized Hamiltonian $g(\theta, \varphi)$ can be viewed in two different ways: it not only defines an interpolation between $h(\theta)$ and $h^{\prime}(\theta)$, but also defines an interpolation between $g(0, \varphi)=h_{1}$ and $g(\pi, \varphi)=h_{2}$. Since $g(0, \varphi)$ and $g(\pi, \varphi)$ are "vacuum Hamiltonians" without any $\varphi$ dependence, they have trivial relative Chern parity, which means $N_{1}\left[h(\theta), h^{\prime}(\theta)\right]=N_{1}[g(0, \varphi), g(\pi, \varphi)]=N_{1}\left[h_{1}, h_{2}\right]=1$.

In conclusion, from the discussion above we have proved that any two interpolations $h(\theta)$ and $h^{\prime}(\theta)$ belong to the same $Z_{2}$ class, so that the Chern parity $N_{1}[h(\theta)]$ only depends on the end points $h_{1}$ and $h_{2}$. Consequently, the quantity $N_{0}\left[h_{1}, h_{2}\right] \equiv N_{1}[h(\theta)]$ defines a relation between each pair of particle-hole symmetric Hamiltonians $h_{1}$ and $h_{2}$. After picking a reference Hamiltonian $h_{0}$, one can define all the Hamiltonians with $N_{0}\left[h_{0}, h\right]=1$ as "trivial" and $N_{0}\left[h_{0}, h\right]=-1$ as nontrivial. The main difference between this classification and the one for $(1+1)$-d systems is that there is no natural choice of the reference Hamiltonian $h_{0}$. In other words, the names "trivial" and "non-trivial" only have relative meaning in the $(0+1)$-d case. However, the classification is still meaningful in the sense that any two Hamiltonians with $N_{0}\left[h_{1}, h_{2}\right]=-1$ cannot be adiabatically connected without breaking particle-hole symmetry. In other words, the manifold of single-site particle-hole symmetric Hamiltonians is disconnected, with at least two connected pieces.

As a simple example, we study $2 \times 2$ Hamiltonians. A general $2 \times 2$ single-site Hamiltonian can be decomposed as

$$
h=d_{0} \sigma^{0}+\sum_{a=1}^{3} d_{a} \sigma^{a}
$$

where $\sigma^{0}=\mathbb{I}$ and $\sigma^{1,2,3}$ are the Pauli matrices. When

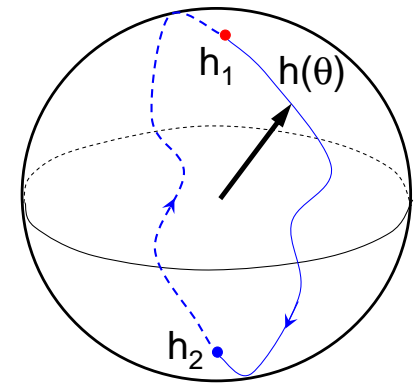

FIG. 6: Illustration of the $2 \times 2$ single-site Hamiltonians. Each point on the sphere represents an unit vector $\hat{\mathbf{d}}=\vec{d} /|d|$, and the north and south poles correspond to the particle-hole symmetric Hamiltonians $h_{1,2}= \pm \sigma^{3}$, respectively. The blue path shows an interpolation between $h_{1}$ and $h_{2}$ satisfying the constraint (46), which always encloses a solid angle $\Omega=2 \pi$.

$C=\sigma^{1}$, particle-hole symmetry requires $C^{\dagger} h C=-h^{T}$, from which we obtain $d_{0}=d_{1}=d_{2}=0$. Thus $h=d_{3} \sigma^{3}$, in which $d_{3} \neq 0$ so as to make $h$ gapped. Consequently, we can see that the two $Z_{2}$ classes are simply $d_{3}>0$ and $d_{3}<0$. When an adiabatic interpolation $h(\theta)=d_{0}(\theta) \sigma^{0}+\sum_{a} d_{a}(\theta) \sigma^{a}$ is defined from $d_{3}>0$ to $d_{3}<0$, the spin vector $\vec{d}(\theta)$ has to rotate from the north pole to the south pole, and then return along the image path determined by the particle-hole symmetry (46), as shown in Fig. 6. The topological quantum number $N_{0}\left[h_{1}, h_{2}\right]$ is simply determined by the Berry's phase enclosed by the path $d_{a}(\theta)$, which is $\pi$ when $h_{1}$ and $h_{2}$ are on different poles, and 0 otherwise. From this example we can understand the $Z_{2}$ classification intuitively. In Sec. $\mathrm{VB}$ we show that the $Z_{2}$ classification of $(2+1)$-d TRI insulators - the class that corresponds to the QSH effect - is obtained as a direct analog of the $(0+1)$ - $d$ case discussed above.

\section{SECOND CHERN NUMBER AND ITS PHYSICAL CONSEQUENCES}

In this section, we shall generalize the classification of the $(2+1)$-d TRB topological insulator in terms of the first Chern number and the $(2+1)$-d Chern-Simons theory to the classification of the $(4+1)$-d TRI topological insulator in terms of the second Chern number and the $(4+1)$-d Chern-Simons theory. We then generalize the dimensional reduction chain $(2+1)-\mathrm{d} \rightarrow(1+1)-\mathrm{d} \rightarrow(0+1)$ $\mathrm{d}$ to the case of $(4+1)-\mathrm{d} \rightarrow(3+1)-\mathrm{d} \rightarrow(2+1)$-d for TRI insulators. Many novel topological effects are predicted for the TRI topological insulators in $(3+1)$ - $d$ and $(2+1)$ d. 


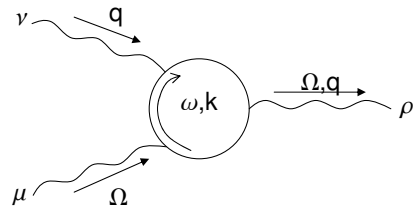

FIG. 7: The Feynman diagram that contributes to the topological term (52). The loop is a fermion propagator, and the wavy lines are external legs corresponding to the gauge field.

\section{A. Second Chern number in $(4+1)$-d non-linear response}

In this subsection, we will show how the second Chern number appears as a non-linear response coefficient of $(4+1)-d$ band insulators in an external $U(1)$ gauge field, which is in exact analogy with the first Chern number as the Hall conductance of a $(2+1)$-d system. To describe such a non-linear response, it is convenient to use the path integral formalism. The Hamiltonian of a $(4+1)$-d insulator coupled to a $U(1)$ gauge field is written as

$$
\begin{aligned}
H[A]= & \sum_{m, n}\left(c_{m \alpha}^{\dagger} h_{m n}^{\alpha \beta} e^{i A_{m n}} c_{n \beta}+\text { h.c. }\right) \\
& +\sum_{m} A_{0 m} c_{m \alpha}^{\dagger} c_{m \alpha} .
\end{aligned}
$$

The effective action of gauge field $A^{\mu}$ is obtained by the following path integral:

$$
e^{i S_{\mathrm{eff}}[A]}=\int D[c] D\left[c^{\dagger}\right] e^{i \int d t\left[\sum_{m} c_{m \alpha}^{\dagger}\left(i \partial_{t}\right) c_{m \alpha}-H[A]\right]}
$$

$$
=\operatorname{det}\left[\left(i \partial_{t}-A_{0 m}\right) \delta_{m n}^{\alpha \beta}-h_{m n}^{\alpha \beta} e^{i A_{m n}}\right]
$$

which determines the response of the fermionic system through the equation

$$
j_{\mu}(\mathbf{x})=\frac{\delta S_{\mathrm{eff}}[A]}{\delta A_{\mu}(\mathbf{x})}
$$

In the case of the $(2+1)$-d insulators, the effective action $S_{\text {eff }}$ contains a Chern-Simons term $\left(C_{1} / 4 \pi\right) A_{\mu} \epsilon^{\mu \nu \tau} \partial_{\nu} A_{\tau}$ as shown in Eq. (9) of Sec. II A in which the first Chern number $C_{1}$ appears as the coefficient. For the $(4+1)$-d system, a similar topological term is in general present in the effective action, which is the second Chern-Simons term:

$$
S_{\mathrm{eff}}=\frac{C_{2}}{24 \pi^{2}} \int d^{4} x d t \epsilon^{\mu \nu \rho \sigma \tau} A_{\mu} \partial_{\nu} A_{\rho} \partial_{\sigma} A_{\tau}
$$

where $\mu, \nu, \rho, \sigma, \tau=0,1,2,3,4$. As shown in Refs. 33. 40, 41, the coefficient $C_{2}$ can be obtained by the one-loop Feynman diagram in Fig. 7, which can be expressed in the following symmetric form:

$$
C_{2}=-\frac{\pi^{2}}{15} \epsilon^{\mu \nu \rho \sigma \tau} \int \frac{d^{4} k d \omega}{(2 \pi)^{5}} \operatorname{Tr}\left[\left(G \frac{\partial G^{-1}}{\partial q^{\mu}}\right)\left(G \frac{\partial G^{-1}}{\partial q^{\nu}}\right)\left(G \frac{\partial G^{-1}}{\partial q^{\rho}}\right)\left(G \frac{\partial G^{-1}}{\partial q^{\sigma}}\right)\left(G \frac{\partial G^{-1}}{\partial q^{\tau}}\right)\right]
$$

in which $q^{\mu}=\left(\omega, k_{1}, k_{2}, k_{3}, k_{4}\right)$ is the frequencymomentum vector, and $G\left(q^{\mu}\right)=\left[\omega+i \delta-h\left(k_{i}\right)\right]^{-1}$ is the single-particle Green's function.

Now we are going to show the relation between $C_{2}$ defined in Eq. (53) and the non-abelian Berry's phase gauge field in momentum space. To make the statement clear, we first write down the conclusion:

- For any $(4+1)$-d band insulator with single particle Hamiltonian $h(\mathbf{k})$, the non-linear response coefficient $C_{2}$ defined in Eq. (53) is equal to the second Chern number of the non-abelian Berry's phase gauge field in the BZ, i.e.:

$$
\begin{aligned}
C_{2} & =\frac{1}{32 \pi^{2}} \int d^{4} k \epsilon^{i j k \ell} \operatorname{tr}\left[f_{i j} f_{k \ell}\right] \\
\text { with } f_{i j}^{\alpha \beta} & =\partial_{i} a_{j}^{\alpha \beta}-\partial_{j} a_{i}^{\alpha \beta}+i\left[a_{i}, a_{j}\right]^{\alpha \beta},
\end{aligned}
$$

$$
a_{i}^{\alpha \beta}(\mathbf{k})=-i\left\langle\alpha, \mathbf{k}\left|\frac{\partial}{\partial k_{i}}\right| \beta, \mathbf{k}\right\rangle
$$

where $i, j, k, \ell=1,2,3,4$.

The index $\alpha$ in $a_{i}^{\alpha \beta}$ refers to the occupied bands, therefore, for a general multi-band model, $a_{i}^{\alpha \beta}$ is a non-abelian gauge field, and $f_{i j}^{\alpha \beta}$ is the associated non-abelian field strength. Here we sketch the basic idea of Eq. (54), and leave the explicit derivation to Appendix B. The key point to simplify Eq. (53) is noticing its topological invariance i.e. under any continuous deformation of the Hamiltonian $h(\mathbf{k})$, as long as no level crossing occurs at the Fermi level, $C_{2}$ remains invariant. Denote the eigenvalues of the single particle Hamiltonian $h(\mathbf{k})$ as $\epsilon_{\alpha}(\mathbf{k}), \alpha=1,2, \ldots, N$ with $\epsilon_{\alpha}(\mathbf{k}) \leq \epsilon_{\alpha+1}(\mathbf{k})$. When $M$ bands are filled, one can always define a continuous de- 


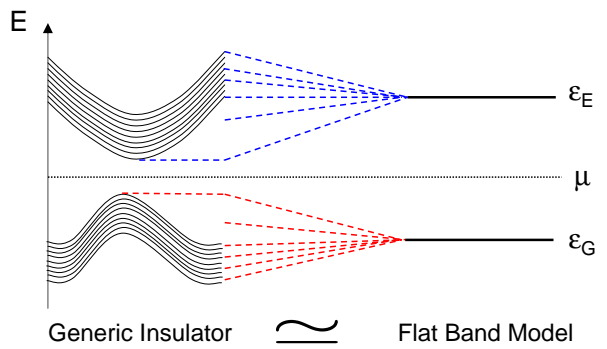

FIG. 8: Illustration showing that a band insulator with arbitrary band structure $\epsilon_{i}(k)$ can be continuously deformed to a flat band model with the same eigenstates. Since no level crossing occurs at the Fermi level, the two Hamiltonians are topologically equivalent.

formation of the energy spectrum so that $\epsilon_{\alpha}(\mathbf{k}) \rightarrow \epsilon_{G}$ for $\alpha \leq M$ and $\epsilon_{\alpha}(\mathbf{k}) \rightarrow \epsilon_{E}$ for $\alpha>M$ (with $\epsilon_{E}>\epsilon_{G}$ ), while all the corresponding eigenstates $|\alpha, \mathbf{k}\rangle$ remain invariant. In other words, each Hamiltonian $h(\mathbf{k})$ can be continuously deformed to some "flat band" model, as shown in Fig. 8. Since both Eq. (53) and the second Chern number in Eq. (54) are topologically invariant, we only need to demonstrate Eq. (54) for the flat band models, of which the Hamiltonians have the form

$$
\begin{aligned}
h_{0}(\mathbf{k}) & =\epsilon_{G} \sum_{1 \leq \alpha \leq M}|\alpha, \mathbf{k}\rangle\left\langle\alpha, \mathbf{k}\left|+\epsilon_{E} \sum_{\beta>M}\right| \beta, \mathbf{k}\right\rangle\langle\beta, \mathbf{k}| \\
& \equiv \epsilon_{G} P_{G}(\mathbf{k})+\epsilon_{E} P_{E}(\mathbf{k}) .
\end{aligned}
$$

Here $P_{G}(\mathbf{k})\left(P_{E}(\mathbf{k})\right)$ is the projection operator to the occupied (un-occupied) bands. Non-abelian gauge connections can be defined in terms of these projection operators in a way similar to Ref. 42. Correspondingly, the single particle Green's function can also be expressed by the projection operators $P_{G}, P_{E}$, and Eq. (54) can be proved by straight-forward algebraic calculations, as shown in Appendix B.

In summary, we have shown that for any $(4+1)$-d band insulator, there is a $(4+1)$-d Chern-Simons term (52) in the effective action of the external $U(1)$ gauge field, of which the coefficient is the second Chern number of the non-abelian Berry phase gauge field. Such a relation between Chern number and Chern-Simons term in the effective action is an exact analogy of the TKNN formula in $(2+1)$-d QH effect. By applying the equation of motion (51), we obtain

$$
j^{\mu}=\frac{C_{2}}{8 \pi^{2}} \epsilon^{\mu \nu \rho \sigma \tau} \partial_{\nu} A_{\rho} \partial_{\sigma} A_{\tau}
$$

which is the non-linear response to the external field $A_{\mu}$. For example, consider a field configuration :

$$
A_{x}=0, A_{y}=B_{z} x, A_{z}=-E_{z} t, A_{w}=A_{t}=0
$$

where $x, y, z, w$ are the spatial coordinates and $t$ is time. The only non-vanishing components of the field curvature are $F_{x y}=B_{z}$ and $F_{z t}=-E_{z}$, which according to Eq. (56) generates the current

$$
j_{w}=\frac{C_{2}}{4 \pi^{2}} B_{z} E_{z} .
$$

If we integrate the equation above over the $x, y$ dimensions (with periodic boundary conditions and assuming $E_{z}$ is does not depend on $\left.(x, y)\right)$, we obtain

$$
\int d x d y j_{w}=\frac{C_{2}}{4 \pi^{2}}\left(\int d x d y B_{z}\right) E_{z} \equiv \frac{C_{2} N_{x y}}{2 \pi} E_{z}
$$

where $N_{x y}=\int d x d y B_{z} / 2 \pi$ is the number of flux quanta through the $x y$ plane, which is always quantized to be an integer. This is exactly the $4 \mathrm{DQH}$ effect proposed in Ref. 14. Thus, from this example we can understand a physical consequence of the second Chern number: In a $(4+1)$-d insulator with second Chern number $C_{2}$, a quantized Hall conductance $C_{2} N_{x y} / 2 \pi$ in the $z w$ plane is induced by magnetic field with flux $2 \pi N_{x y}$ in the perpendicular $(x y)$ plane.

Similar to the $(2+1)$-d case, the physical consequences of the second Chern number can also be understood better by studying the surface states of an open-boundary system, which for the $(4+1)$-d case is described by a $(3+1)$-d theory. In the next subsection we will study an explicit example of a $(4+1)$-d topological insulator, which helps us to improve our understanding of the physical picture of the $(4+1)$-d topology; especially, after dimensional reduction to the lower-dimensional physical systems.

\section{B. TRI topological insulators based on lattice Dirac models}

In section IIB we have shown that the model introduced in Ref. 35 realizes the fundamental TRB topological insulator in $(2+1)$-d, and it reduces to the Dirac model in the continuum limit. Generalizing this construction, we propose the lattice Dirac model to be the realization of the fundamental TRI topological insulator in $(4+1)$-d. Such a model has also been studied in the field theory literature ${ }^{40,43}$. The continuum Dirac model in $(4+1)$ - $d$ dimensions is expressed as

$$
H=\int d^{4} x\left[\psi^{\dagger}(x) \Gamma^{i}\left(-i \partial_{i}\right) \psi(x)+m \psi^{\dagger} \Gamma^{0} \psi\right]
$$

with $i=1,2,3,4$ the spatial dimensions, and $\Gamma^{\mu}, \mu=$ $0,1, . ., 4$ the five Dirac matrices satisfying the Clifford algebra

$$
\left\{\Gamma^{\mu}, \Gamma^{\nu}\right\}=2 \delta_{\mu \nu} \mathbb{I}
$$

with $\mathbb{I}$ the identity matrix ${ }^{44}$.

The lattice (tight-binding) version of this model is written as

$$
H=\sum_{n, i}\left[\psi_{n}^{\dagger}\left(\frac{c \Gamma^{0}-i \Gamma^{i}}{2}\right) \psi_{n+\hat{i}}+\text { h.c. }\right]
$$




$$
+m \sum_{n} \psi_{n}^{\dagger} \Gamma^{0} \psi_{n}
$$

or in momentum space,

$$
H=\sum_{\mathbf{k}} \psi_{\mathbf{k}}^{\dagger}\left[\sum_{i} \sin k_{i} \Gamma^{i}+\left(m+c \sum_{i} \cos k_{i}\right) \Gamma^{0}\right] \psi_{\mathbf{k}}
$$

Such a Hamiltonian can be written in the compact form

$$
H=\sum_{\mathbf{k}} \psi_{\mathbf{k}}^{\dagger} d_{a}(\mathbf{k}) \Gamma^{a} \psi_{\mathbf{k}}
$$

with

$d_{a}(\mathbf{k})=\left(\left(m+c \sum_{i} \cos k_{i}\right), \sin k_{x}, \sin k_{y}, \sin k_{z}, \sin k_{w}\right)$

a five-dimensional vector. Similar to the $(2+1)$-d twoband models we studied in Sec. IIB, a single particle Hamiltonian with the form $h(\mathbf{k})=d_{a}(\mathbf{k}) \Gamma^{a}$ has two eigenvalues $E_{ \pm}(\mathbf{k})= \pm \sqrt{\sum_{a} d_{a}^{2}(\mathbf{k})}$, but with the key difference that here both eigenvalues are doubly degenerate. When $\sum_{a} d_{a}^{2}(\mathbf{k}) \equiv d^{2}(\mathbf{k})$ is non-vanishing in the whole $\mathrm{BZ}$, the system is gapped at half-filling, with the two bands with $E=E_{-}(\mathbf{k})$ filled. Since there are two occupied bands, an $S U(2) \times U(1)$ adiabatic connection can be defined $42,45,46$. Starting from the Hamiltonian (62), one can determine the single particle Green's function, and substituting it into the expression for the second Chern number in Eq. (53). We obtain

$$
C_{2}=\frac{3}{8 \pi^{2}} \int d^{4} k \epsilon^{a b c d e} \hat{d}_{a} \partial_{x} \hat{d}_{b} \partial_{y} \hat{d}_{c} \partial_{z} \hat{d}_{d} \partial_{w} \hat{d}_{e}
$$

which is the winding number of the mapping $\hat{d}_{a}(\mathbf{k}) \equiv$ $d_{a}(\mathbf{k}) /|d(\mathbf{k})|$ from the BZ $T^{4}$ to the sphere $S^{4}$ and $a, b, c, d, e=0,1,2,3,4$. More details of this calculation are presented in Appendix $\mathrm{C}$.

Since the winding number (64) is equal to the second Chern number of the Berry's phase gauge field, it is topologically invariant. It is easy to calculate $C_{2}$ in the lattice Dirac model (61). Considering the lattice Dirac model with a fixed positive parameter $c$ and tunable mass term $m, C_{2}(m)$ as a function of $m$ can change only if the Hamiltonian is gapless, i.e., if $\sum_{a} d_{a}^{2}(\mathbf{k}, m)=0$ for some k. It's easy to determine that $C_{2}(m)=0$ in the limit $m \rightarrow+\infty$, since the unit vector $\hat{d}_{a}(\mathbf{k}) \rightarrow(1,0,0,0,0)$ in that limit. Thus we only need to study the change of $C_{2}(m)$ at each quantum critical points, namely at critical values of $m$ where the system becomes gapless.

The solutions of equation $\sum_{a} d_{a}^{2}(\mathbf{k}, m)=0$ lead to five critical values of $m$ and corresponding $\mathbf{k}$ points as listed below:

$$
m=\left\{\begin{array}{cc}
-4 c, \quad \mathbf{k}=(0,0,0,0) \\
-2 c, \quad \mathbf{k} \in P[(\pi, 0,0,0)] \\
0, & \mathbf{k} \in P[(\pi, 0, \pi, 0)] \\
2 c, & \mathbf{k} \in P[(\pi, \pi, \pi, 0)] \\
4 c, & \mathbf{k}=(\pi, \pi, \pi, \pi)
\end{array}\right.
$$

in which $P[\mathbf{k}]$ stands for the set of all the wavevectors obtained from index permutations of wavevector $\mathbf{k}$. For example, $P[(\pi, 0,0,0)]$ consists of $(\pi, 0,0,0),(0, \pi, 0,0)$, $(0,0, \pi, 0)$ and $(0,0,0, \pi)$. As an example, we can study the change of $C_{2}(m)$ around the critical value $m=-4 c$. In the limit $m+4 c \ll 2 c$, the system has its minimal gap at $\mathbf{k}=\mathbf{0}$, around which the $d_{a}(\mathbf{k})$ vector has the approximate form $d_{a}(\mathbf{k}) \simeq\left(\delta m, k_{x}, k_{y}, k_{z}, k_{w}\right)+o(|k|)$, with $\delta m \equiv m+4 c$. Taking a cut-off $\Lambda \ll 2 \pi$ in momentum space, one can divide the expression (64) of $C_{2}$ into low-energy and high-energy parts:

$$
C_{2}=\frac{3}{8 \pi^{2}}\left(\int_{|\mathbf{k}| \leq \Lambda} d^{4} k+\int_{|\mathbf{k}|>\Lambda} d^{4} k\right) \epsilon^{a b c d e} \hat{d}_{a} \partial_{x} \hat{d}_{b} \partial_{y} \hat{d}_{c} \partial_{z} \hat{d}_{d} \partial_{w} \hat{d}_{e} \equiv C_{2}^{(1)}(\delta m, \Lambda)+C_{2}^{(2)}(\delta m, \Lambda)
$$

Since there is no level-crossing in the region $|\mathbf{k}|>\Lambda$, the jump of $C_{2}$ at $\delta m=0$ can only come from $C_{2}^{(1)}$. In the limit $|\delta m|<\Lambda \ll 2 \pi$, the continuum approximation of $d_{a}(\mathbf{k})$ can be applied to obtain

$$
C_{2}^{(1)}(\delta m, \Lambda) \simeq \frac{3}{8 \pi^{2}} \int_{|\mathbf{k}| \leq \Lambda} d^{4} k \frac{\delta m}{\left(\delta m^{2}+\mathbf{k}^{2}\right)^{5 / 2}}
$$

which can be integrated and leads to

$$
\Delta C_{2 \delta m=0^{-}}^{\delta m=0^{+}}=\Delta C_{2}^{(1)} \begin{gathered}
\delta m=0^{+} \\
\delta m=0^{-}
\end{gathered}=1 .
$$

From the analysis above we see that the change of the second Chern number is determined only by the effective continuum model around the level crossing wavevector(s). In this case the continuum model is just the Dirac model. Similar analysis can be carried out at the other critical $m$ 's, which leads to the following values of the 
second Chern number:

$$
C_{2}(m)=\left\{\begin{array}{cc}
0, & m<-4 c \text { or } m>4 c \\
1, & -4 c<m<-2 c \\
-3, & -2 c<m<0 \\
3, & 0<m<2 c \\
-1, & 2 c<m<4 c
\end{array} .\right.
$$

A more general formula is given in Ref. 40 .

After obtaining the second Chern number, we can study the surface states of the topologically nontrivial phases of this model. In the same way as in Sec. [IC] we can take open boundary conditions for one dimension, say, $w$, and periodic boundary conditions for all other dimensions, so that $k_{x}, k_{y}, k_{z}$ are still good quantum numbers. The Hamiltonian is transformed to a sum of $1 \mathrm{D}$ tight-binding models:

$$
\begin{aligned}
H & =\sum_{\vec{k}, w}\left[\psi_{\vec{k}}^{\dagger}(w)\left(\frac{c \Gamma^{0}-i \Gamma^{4}}{2}\right) \psi_{\vec{k}}(w+1)+h . c .\right] \\
& +\sum_{\vec{k}, w} \psi_{\vec{k}}^{\dagger}(w)\left[\sin k_{i} \Gamma^{i}+\left(m+c \sum_{i} \cos k_{i}\right) \Gamma^{0}\right] \psi_{\vec{k}}(w)
\end{aligned}
$$

in which $\vec{k}=\left(k_{x}, k_{y}, k_{z}\right), i=1,2,3$, and $w=1,2, . ., L$ are the $w$ coordinates of lattice sites. The single-particle energy spectrum can be obtained as $E_{\alpha}(\vec{k}), \alpha=1,2, . .4 L$, among which the mid-gap surface states are found when $C_{2} \neq 0$, as shown in Fig. 9. When the Chern number is $C_{2}$, there are $\left|C_{2}\right|$ branches of gapless surface states with linear dispersion, so that the low energy effective theory is described by $\left|C_{2}\right|$ flavors of chiral fermions ${ }^{43}$ :

$$
H=\operatorname{sgn}\left(C_{2}\right) \int \frac{d^{3} p}{(2 \pi)^{3}} \sum_{i=1}^{\left|C_{2}\right|} v_{i} \psi_{i}^{\dagger}(\vec{p}) \vec{\sigma} \cdot \overrightarrow{\mathbf{p}} \psi_{i}(\vec{p}) .
$$

The factor $\operatorname{sgn}\left(C_{2}\right)$ ensures that the chirality of the surface states is determined by the sign of the Chern number. From such a surface theory we can obtain a more physical understanding of the nonlinear response equation (56) to an external $U(1)$ gauge field. Taking the same gauge field configuration as in Eq. (57), the nonvanishing components of the field curvature are $F_{x y}=B_{z}$ and $F_{z t}=-E_{z}$. Consequently, the $(3+1)$-d surface states are coupled to a magnetic field $\mathbf{B}=B_{z} \hat{\mathbf{z}}$ and an electric field $\mathbf{E}=E_{z} \hat{\mathbf{z}}$. For simplicity, consider the system with $-4 c<m<-2 c$ and $C_{2}=1$, in which the surface theory is a single chiral fermion with the single particle Hamiltonian

$h=v \vec{\sigma} \cdot(\vec{p}+\vec{A})=v \sigma_{x} p_{x}+v \sigma_{y}\left(p_{y}+B_{z} x\right)+v \sigma_{z}\left(p_{z}-E_{z} t\right)$.

If $E_{z}$ is small enough so that the time-dependence of $A_{z}(t)=-E_{z} t$ can be treated adiabatically, the single particle energy spectrum can be solved for a fixed $A_{z}$ as

$$
\begin{aligned}
E_{n \pm}\left(p_{z}\right) & = \pm v \sqrt{\left(p_{z}+A_{z}\right)^{2}+2 n\left|B_{z}\right|}, n=1,2, \ldots \\
E_{0}\left(p_{z}\right) & =v\left(p_{z}+A_{z}\right) \operatorname{sgn}\left(B_{z}\right) .
\end{aligned}
$$
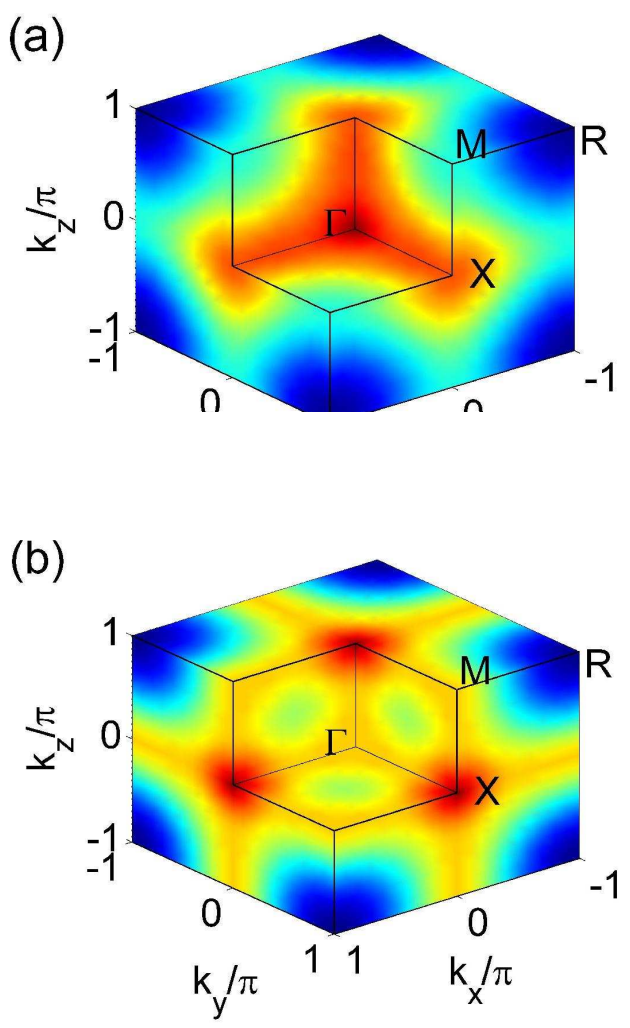

FIG. 9: Three dimensional energy spectrum of the surface states for the parameters (a) $c=1, m=-3$ and (b) $c=$ $1, m=-1$. The Dirac points where energy gap vanishes are marked with deepest red color. For $m=-3$ there is one Dirac point at $\Gamma$ point while for $m=-1$ there are three of them at $X$ points.

When the size of the surface is taken as $L_{x} \times L_{y} \times L_{z}$ with periodic boundary conditions, each Landau level has the degeneracy $N_{x y}=L_{x} L_{y} B_{z} / 2 \pi$. Similar to Laughlin's gauge argument for QH edge states ${ }^{4}$, the effect of an infinitesimal electric field $E_{z}$ can be obtained by adiabatically shifting the momentum $p_{z} \rightarrow p_{z}+E_{z} t$. As shown in Fig. 10, from the time $t=0$ to $t=T \equiv 2 \pi / L_{z} E_{z}$, the momentum is shifted as $p_{z} \rightarrow p_{z}+2 \pi / L_{z}$, so that the net electron number of the surface 3D system increases by $N_{x y}$. In other words, a "generalized Hall current" $I_{w}$ must be flowing towards the $w$ direction:

$$
I_{w}=\frac{N_{x y}}{T}=\frac{L_{x} L_{y} L_{z} B_{z} E_{z}}{4 \pi^{2}} .
$$

This "generalized Hall current" is the key property of the 4DQH effect studied in Ref. 14 .

In terms of current density we obtain $j_{w}=B_{z} E_{z} / 4 \pi^{2}$, which is consistent with the result of Eq. (56) discussed in the last subsection. More generally the current density $j_{w}$ can be written as

$$
j_{w}=C_{2} \frac{\mathbf{E} \cdot \mathbf{B}}{4 \pi^{2}}=\frac{C_{2}}{32 \pi^{2}} \epsilon^{\mu \nu \sigma \tau} F_{\mu \nu} F_{\sigma \tau}
$$

which is the chiral anomaly equation of massless $(3+1)-\mathrm{d}$ Dirac fermions $\frac{47,48}{2}$. Since the gapless states on the 3D 


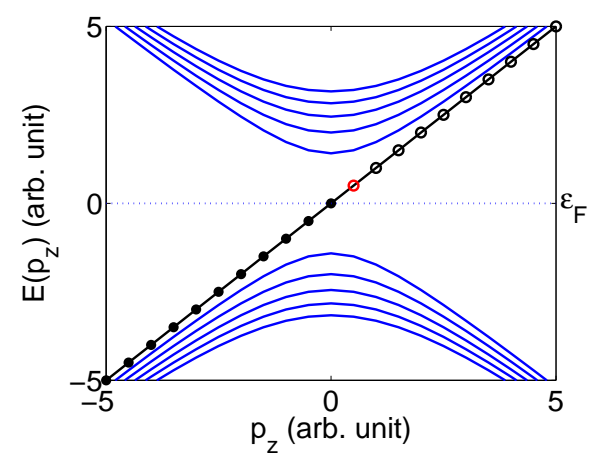

FIG. 10: Illustration of the surface Landau level spectrum given by Eq. (70). Each level in the figure is $N_{x y}$ fold degenerate. The solid circles are the occupied states of the zeroth Landau level, and the red open circle shows the states that are filled when the gauge vector potential $A_{z}$ is shifted adiabatically from 0 to $2 \pi / L_{z}$.

edge of the 4D lattice Dirac model are chiral fermions, the current $I_{w}$ carries away chiral charge, leading to the non-conservation of chirality on the 3D edge.

\section{DIMENSIONAL REDUCTION TO $(3+1)-\mathbf{D}$ TRI INSULATORS}

As shown in Sec. IIC, one can start from a $(2+1)$ d TRB topological insulator described by a Hamiltonian $h\left(k_{x}, k_{y}\right)$, and perform the procedure of dimensional reduction by replacing $k_{y}$ by a parameter $\theta$. The same dimensional reduction procedure can be carried out for the $(4+1)$-d TRI insulator with a non-vanishing second Chern number. From this procedure, one obtains the topological effective theory of insulators in $(3+1)$-d and $(2+1)$-d. Specifically, for TRI insulators a general $Z_{2}$ topological classification is defined. Compared to the earlier proposals of the $Z_{2}$ topological invariant $16,22,23,24,27,28,29,30$, our approach provides a direct relationship between the topological quantum number and the physically measurable topological response of the corresponding system. We discuss a number of theoretical predictions, including the TME effect, and propose experimental settings where these topological effects can be measured in realistic materials.

\section{A. Effective action of $(3+1)$-d insulators}

To perform the dimensional reduction explicitly, in the following we show the derivation for the $(4+1)$-d Dirac model (61). However, each step of the derivation is applicable to any other insulator model, so the conclusion is completely generic.

The Hamiltonian of Dirac model (61) coupled to an external $U(1)$ gauge field is given by

$$
H[A]=\sum_{n, i}\left[\psi_{n}^{\dagger}\left(\frac{c \Gamma^{0}-i \Gamma^{i}}{2}\right) e^{i A_{n, n+\hat{\imath}}} \psi_{n+\hat{i}}+\text { h.c. }\right]
$$

$$
+m \sum_{n} \psi_{n}^{\dagger} \Gamma^{0} \psi_{n}
$$

Now consider a special "Landau"-gauge configuration satisfying $A_{n, n+\hat{i}}=A_{n+\hat{w}, n+\hat{w}+\hat{i}}, \forall n$, which is translationally invariant in the $w$ direction. Thus, under periodic boundary conditions the $w$-direction momentum $k_{w}$ is a good quantum number, and the Hamiltonian can be rewritten as

$$
\begin{aligned}
H[A]= & \sum_{k_{w}, \vec{x}, s}\left[\psi_{\vec{x}, k_{w}}^{\dagger}\left(\frac{c \Gamma^{0}-i \Gamma^{s}}{2}\right) e^{i A_{\vec{x}, \vec{x}+\hat{s}}} \psi_{\vec{x}+\hat{s}, k_{w}}+h . c .\right] \\
& +\sum_{k_{w}, \vec{x}, s} \psi_{\vec{x}, k_{w}}^{\dagger}\left[\sin \left(k_{w}+A_{\vec{x} 4}\right) \Gamma^{4}\right. \\
& \left.+\left(m+c \cos \left(k_{w}+A_{\vec{x} 4}\right)\right) \Gamma^{0}\right] \psi_{\vec{x}, k_{w}}
\end{aligned}
$$

where $\vec{x}$ stands for the three-dimensional coordinates, $A_{\vec{x} 4} \equiv A_{\vec{x}, \vec{x}+\hat{w}}$, and $s=1,2,3$ stands for the $x, y, z$ directions. In this expression, the states with different $k_{w}$ decouple from each other, and the $(4+1)$-d Hamiltonian $H[A]$ reduces to a series of $(3+1)$-d Hamiltonians. Pick one of these $(3+1)$-d Hamiltonians with fixed $k_{w}$ and rename $k_{w}+A_{\vec{x} 4}=\theta_{\vec{x}}$, we obtain the $(3+1)$-d model

$$
\begin{aligned}
H_{3 \mathrm{D}}[A, \theta]= & \sum_{\vec{x}, s}\left[\psi_{\vec{x}}^{\dagger}\left(\frac{c \Gamma^{0}-i \Gamma^{s}}{2}\right) e^{i A_{\vec{x}, \vec{x}+\hat{s}}} \psi_{\vec{x}+\hat{s}}+h . c .\right] \\
& +\sum_{\vec{x}, s} \psi_{\vec{x}}^{\dagger}\left[\sin \theta_{\vec{x}} \Gamma^{4}+\left(m+c \cos \theta_{\vec{x}}\right) \Gamma^{0}\right] \psi_{\vec{x}}
\end{aligned}
$$

which describes a band insulator coupled to an electromagnetic field $A_{\vec{x}, \vec{x}+\hat{s}}$ and an adiabatic parameter field $\theta_{\vec{x}}$.

Due to its construction, the response of the model (73) to $A_{\vec{x}, \vec{x}+\hat{s}}$ and $\theta_{\vec{x}}$ fields is closely related to the response of the $(4+1)$-d Dirac model (61) to the $U(1)$ gauge field. To study the response properties of the $(3+1)$-d system, the effective action $S_{3 \mathrm{D}}[A, \theta]$ can be defined as

$$
\exp ^{i S_{3 \mathrm{D}}[A, \theta]}=\int D[\psi] D[\bar{\psi}] e^{i \int d t\left[\sum_{\vec{x}} \bar{\psi}_{\vec{x}}\left(i \partial_{\tau}-A_{\vec{x} 0}\right) \psi_{\vec{x}}-H[A, \theta]\right]} .
$$

A Taylor expansion of $S_{3 \mathrm{D}}$ can be carried out around the field configuration $A_{s}(\vec{x}, t) \equiv 0, \theta(\vec{x}, t) \equiv \theta_{0}$, which contains a non-linear response term directly derived from the $(4+1)$-d Chern-Simons action (52):

$$
S_{3 \mathrm{D}}=\frac{G_{3}\left(\theta_{0}\right)}{4 \pi} \int d^{3} x d t \epsilon^{\mu \nu \sigma \tau} \delta \theta \partial_{\mu} A_{\nu} \partial_{\sigma} A_{\tau} .
$$

Compared to the Eq. (52), the field $\delta \theta(\vec{x}, t)=\theta(\vec{x}, t)-\theta_{0}$ plays the role of $A_{4}$, and the coefficient $G_{3}\left(\theta_{0}\right)$ is determined by the same Feynman diagram (7), but evaluated for the three-dimensional Hamiltonian (73). Consequently, $G_{3}\left(\theta_{0}\right)$ can be calculated and is equal to Eq. (53), but without the integration over $k_{w}$ : 


$$
G_{3}\left(\theta_{0}\right)=-\frac{\pi}{6} \int \frac{d^{3} k d \omega}{(2 \pi)^{4}} \operatorname{Tr} \epsilon^{\mu \nu \sigma \tau}\left[\left(G \frac{\partial G^{-1}}{\partial q^{\mu}}\right)\left(G \frac{\partial G^{-1}}{\partial q^{\nu}}\right)\left(G \frac{\partial G^{-1}}{\partial q^{\sigma}}\right)\left(G \frac{\partial G^{-1}}{\partial q^{\tau}}\right)\left(G \frac{\partial G^{-1}}{\partial \theta_{0}}\right)\right]
$$

where $q^{\mu}=\left(\omega, k_{x}, k_{y}, k_{z}\right)$. Due to the same calculation as Sec. III A and Appendix B $G_{3}\left(\theta_{0}\right)$ is determined from the Berry phase curvature as

$$
G_{3}\left(\theta_{0}\right)=\frac{1}{8 \pi^{2}} \int d^{3} k \epsilon^{i j k} \operatorname{tr}\left[f_{\theta i} f_{j k}\right]
$$

in which the Berry phase gauge field is defined in the four-dimensional space $\left(k_{x}, k_{y}, k_{z}, \theta_{0}\right)$, i.e., $a_{i}^{\alpha \beta}=-i\left\langle\vec{k}, \theta_{0} ; \alpha\left|\left(\partial / \partial k_{i}\right)\right| \vec{k}, \theta_{0} ; \beta\right\rangle$ and $a_{\theta}^{\alpha \beta}=$ $-i\left\langle\vec{k}, \theta_{0} ; \alpha\left|\left(\partial / \partial \theta_{0}\right)\right| \vec{k}, \theta_{0} ; \beta\right\rangle$. Compared to the second Chern number (54), we know that $G_{3}\left(\theta_{0}\right)$ satisfies the sum rule

$$
\int G_{3}\left(\theta_{0}\right) d \theta_{0}=C_{2} \in \mathbb{Z}
$$

which is in exact analogy with the sum rule of the pumping coefficient $G_{1}(\theta)$ in Eq. (22) of the $(1+1)$-d system. Recall that $G_{1}(\theta)$ can be expressed as $\partial P_{1}(\theta) / \partial \theta$, where $P_{1}(\theta)$ is simply the charge polarization. In comparison, a generalized polarization $P_{3}\left(\theta_{0}\right)$ can also be defined in $(3+1)$-d so that $G_{3}\left(\theta_{0}\right)=\partial P_{3}\left(\theta_{0}\right) / \partial \theta_{0}$. ( Recently, a similar quantity has also been considered in Ref. 49 from the point of view of semiclassical particle dynamics.) The conventional electric polarization $\mathbf{P}$ couples linearly to the external electric field $\mathbf{E}$, and the magnetic polarization $\mathbf{M}$ couples linearly to the magnetic field $\mathbf{B}$, however, as we shall show, $P_{3}$ is a pseudo-scalar which couples nonlinearly to the external electromagnetic field combination E.B. For this reason, we coin the term "magneto-electric polarization" for $P_{3}$. To obtain $P_{3}\left(\theta_{0}\right)$, one needs to introduce the non-Abelian Chern-Simons term:

$$
\mathcal{K}^{A}=\frac{1}{16 \pi^{2}} \epsilon^{A B C D} \operatorname{Tr}\left[\left(f_{B C}-\frac{1}{3}\left[a_{B}, a_{C}\right]\right) \cdot a_{D}\right],
$$

which is a vector in the four-dimensional parameter space $q=\left(k_{x}, k_{y}, k_{z}, \theta_{0}\right)$ and $A, B, C, D=x, y, z, \theta . \mathcal{K}^{A}$ satisfies

$$
\begin{aligned}
\partial_{A} \mathcal{K}^{A} & =\frac{1}{32 \pi^{2}} \epsilon^{A B C D} \operatorname{tr}\left[f_{A B} f_{C D}\right] \\
\Rightarrow G_{3}\left(\theta_{0}\right) & =\int d^{3} k \partial_{A} \mathcal{K}^{A} .
\end{aligned}
$$

When the second Chern number is nonzero, there is an obstruction to the definition of $a_{A}$, which implies that $\mathcal{K}_{A}$ cannot be a single-valued continuous function in the whole parameter space. However, in an appropriate gauge choice, $\mathcal{K}^{i}, i=x, y, z$ can be single-valued, so that $G_{3}\left(\theta_{0}\right)=\int d^{3} k \partial_{\theta} \mathcal{K}^{\theta} \equiv \partial P_{3}\left(\theta_{0}\right) / \partial \theta_{0}$, with

$$
P_{3}\left(\theta_{0}\right)=\int d^{3} k \mathcal{K}^{\theta}
$$

$$
=\frac{1}{16 \pi^{2}} \int d^{3} k \epsilon^{\theta i j k} \operatorname{Tr}\left[\left(f_{i j}-\frac{1}{3}\left[a_{i}, a_{j}\right]\right) \cdot a_{k}\right] .
$$

Thus, $P_{3}\left(\theta_{0}\right)$ is given by the integral of the non-Abelian Chern-Simons 3 -form over momentum space. This is analogous to the charge polarization defined as the integral of the adiabatic connection 1-form over a path in momentum space.

As is well-known, the three-dimensional integration of the Chern-Simons term is only gauge-invariant modulo an integer. Under a gauge transformation $a_{i} \rightarrow u^{-1} a_{i} u-$ $i u^{-1} \partial_{i} u(u \in U(M)$ when $M$ bands are occupied), the change of $P_{3}$ is

$$
\Delta P_{3}=\frac{i}{24 \pi^{2}} \int d^{3} k \epsilon^{\theta i j k} \operatorname{Tr}\left[\left(u^{-1} \partial_{i} u\right)\left(u^{-1} \partial_{j} u\right)\left(u^{-1} \partial_{k} u\right)\right],
$$

which is an integer. Thus $P_{3}\left(\theta_{0}\right)$, just like $P_{1}(\theta)$, is only defined modulo 1 , and its change during a variation of $\theta_{0}$ from 0 to $2 \pi$ is well-defined, and given by $C_{2}$.

The effective action (74) can be further simplified by introducing $G_{3}=\partial P_{3} / \partial \theta$. Integration by parts of $S_{3 \mathrm{D}}$ leads to

$$
S_{3 \mathrm{D}}=\frac{1}{4 \pi} \int d^{3} x d t \epsilon^{\mu \nu \sigma \tau} A_{\mu}\left(\partial P_{3} / \partial \theta\right) \partial_{\nu} \delta \theta \partial_{\sigma} A_{\tau} .
$$

$\left(\partial P_{3} / \partial \theta\right) \partial_{\nu} \delta \theta$ can be written as $\partial_{\nu} P_{3}$, where $P_{3}(\vec{x}, t)=$ $P_{3}(\theta(\vec{x}, t))$ has space-time dependence determined by the $\theta$ field. Such an expression is only meaningful when the space-time dependence of $\theta$ field is smooth and adiabatic, so that locally $\theta$ can still be considered as a parameter. In summary, the effective action is finally written as

$$
S_{3 \mathrm{D}}=\frac{1}{4 \pi} \int d^{3} x d t \epsilon^{\mu \nu \sigma \tau} P_{3}(x, t) \partial_{\mu} A_{\nu} \partial_{\sigma} A_{\tau} .
$$

This effective topological action for the $(3+1)$-d insulator is one of the central results of this paper. As we shall see later, many physical consequences can be directly derived from it. It should be emphasized that this effective action is well-defined for an arbitrary $(3+1)$-d insulator Hamiltonian $h(\vec{k}, \vec{x}, t)$ in which the dependence on $\vec{x}, t$ is adiabatic. We obtained this effective theory by the dimensional reduction from a $(4+1)$-d system; and we presented it this way since we believe that this derivation is both elegant and unifying. However, for readers who are not interested in the relationship to higher dimensional physics, a self-contained derivation can also be carried out directly in $(3+1)$-d, as we explained earlier, by integrating out the fermions in the presence of the $A_{\mu}(x, t)$ and the $\theta(x, t)$ external fields. 
This effective action is known in the field theory literature as axion electrodynamics $50,51,52$, where the adiabatic field $P_{3}$ plays the role of the axion field ${ }^{53,54}$. When the $P_{3}$ field becomes a constant parameter independent of space and time, this effective action is referred to as the topological term for the $\theta$ vacuum 55,56 . The axion field has not yet been experimentally identified, and it remains as a deep mystery in particle physics. Our work shows that the same physics can occur in a condensed matter system, where the adiabatic "axion" field $P_{3}(x, t)$ has a direct physical interpretation and can be accessed and controlled experimentally.

From the discussion above it is clear that 3D TRI topological insulators realize a non-trivial solitonic background $\theta$ field. In Ref. 28 the authors suggest several candidate materials which could be $3 \mathrm{D}$ topological insulators. These 3D materials are topologically non-trivial because of band inversion mechanism similar to that of the HgTe quantum wells ${ }^{20}$. Ref. 57 closely studied the strained, bulk HgTe. We will keep this system in mind since it has a simple physical interpretation, and its essential physics can be described by the Dirac model presented earlier. We can consider the trivial vacuum outside the material to have a constant axion field with the value $\theta=0$ and the interior of a $3 \mathrm{D}$ topological insulator to have a $\theta=\pi$ background field. The value $\theta=\pi$ does not violate time-reversal (or CP in highenergy language). HgTe is a zero-gap semiconductor and has no topologically protected features. However, when strained, the system develops a bulk insulating gap between the p-wave light-hole "conduction band" and the p-wave heavy-hole "valence band" around the $\Gamma$-point. To study the topological features we must also include the s-wave band which in a conventional material like GaAs would be a conduction band. Because of the strong spin-orbit coupling in $\mathrm{HgTe}$ the band structure is actually inverted the s-wave band becomes a valence band. For a moment we will ignore the heavy-hole band and only consider the light-hole and s-wave band ${ }^{57}$. The effective Hamiltonian of these two bands is a massive Dirac Hamiltonian, but with a negative mass. The negative mass indicates a phase shift of $\pi$ in the vacuum angle $\theta$ from its original unshifted value in the trivial vacuum. The axion domain wall structure at the surface of the topological insulator traps fermion zero modes which are simply the topologically protected surface states. If we include the effects of the heavy-hole band the dispersion of the bulk bands and surface states are quantitatively modified. However, as long as the crystal is strained enough to maintain the bulk gap the topological phenomena will be unaffected and the boundary of the 3D topological insulator can still be described as an axion domain wall. Thus, this material in condensed matter physics provide a direct realization of axion electrodynamics.

\section{B. Physical Consequences of the Effective Action $S_{3 D}$}

In this subsection we present the general physical consequences of the effective topological action (80) for $(3+1)$-d insulators coupled to a $P_{3}$ polarization, and in subsection IVC we focus on its consequences for TRI insulators. Since the effective action is quadratic in $A_{\mu}$, it describes a linear response to the external electromagnetic fields which depends on the spatial and temporal gradients of $P_{3}$. Taking a variation of $S_{3 \mathrm{D}}[A, \theta]$ we obtain the response equation:

$$
j^{\mu}=\frac{1}{2 \pi} \epsilon^{\mu \nu \sigma \tau} \partial_{\nu} P_{3} \partial_{\sigma} A_{\tau} .
$$

The physical consequences Eq. (81) can be understood by studying the following two cases.

(1) Hall effect induced by spatial gradient of $\mathbf{P}_{3}$.

Consider a system in which $P_{3}=P_{3}(z)$ only depends on $z$. For example, this can be realized by the lattice Dirac model (73) with $\theta=\theta(z)$. (This type of domain wall has also been considered in Ref. 58). In this case Eq. (81) becomes

$$
j^{\mu}=\frac{\partial_{z} P_{3}}{2 \pi} \epsilon^{\mu \mu \rho} \partial_{\nu} A_{\rho}, \mu, \nu, \rho=t, x, y
$$

which describes a $\mathrm{QH}$ effect in the $x y$ plane with the Hall conductivity $\sigma_{x y}=\partial_{z} P_{3} / 2 \pi$, as shown in Fig. 11 (a). For a uniform electric field $E_{x}$ in the $x$-direction, the Hall current density is $j_{y}=\left(\partial_{z} P_{3} / 2 \pi\right) E_{x}$. Thus the integration over $z$ in a finite range gives the $2 \mathrm{D}$ current density in the $x y$ plane:

$$
J_{y}^{2 \mathrm{D}}=\int_{z_{1}}^{z_{2}} d z j_{y}=\frac{1}{2 \pi}\left(\int_{z_{1}}^{z_{2}} d P_{3}\right) E_{x} .
$$

In other words, the net Hall conductance of the region $z_{1} \leq z \leq z_{2}$ is

$$
\sigma_{x y}^{2 \mathrm{D}}=\int_{z_{1}}^{z_{2}} d P_{3} / 2 \pi
$$

which only depends on the change of $P_{3}$ in this region, and is not sensitive to any details of the function $P_{3}(z)$. Analogously in the $(1+1)-d$ case, if we perform the spatial integration of Eq. (25), we obtain the total charge induced by the charge polarization $P$ :

$$
Q=-\int_{z_{1}}^{z_{2}} d P / 2 \pi
$$

By comparing these two equations, we see that the relation between $P_{3}$ and Hall conductance in $(3+1)$-d insulators is the same as the relation between charge polarization $P$ and the total charge in the $(1+1)$-d case. As a specific case, a domain wall between two homogeneous materials with different $P_{3}$ will carry Hall conductance $\sigma_{H}=\Delta P_{3} / 2 \pi$, while the fractional charge carried by a domain wall in $(1+1)$-d is given by $Q=-\Delta P / 2 \pi$. 

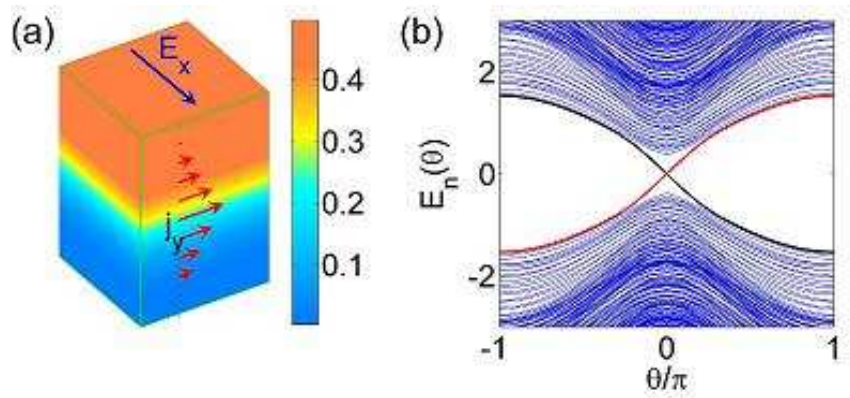

FIG. 11: (a) Illustration of the Hall effect induced by a spatial gradient of $P_{3}$. The colors represent different values of $P_{3}$, which decrease from 0 at the bottom to $1 / 2$ on top. The blue arrow shows the direction of a uniform electric field $E_{x}$ and the white arrows show the Hall current density induced, given by the formula $j_{y}=\left(\partial_{z} P_{3} / 2 \pi\right) E_{x}$. (b) Energy spectrum of the $(3+1)$-d lattice Dirac model (73) in a magnetic field $B_{z}$ towards the $z$ direction. The boundary conditions are periodic in the $x$ and $y$-directions and open on the $z$-direction. The red and black curves show the surface states on the top and bottom surfaces, respectively, each of which is a $B_{z} L_{x} L_{y} / 2 \pi$ fold degenerate Landau Level. The parameters of model (73) are chosen to be $m=-3, c=1$.

(2) Topological Magneto-electric effect(TME) induced by temporal gradient of $\mathbf{P}_{3}$.

When $P_{3}=P_{3}(t)$ is spatially uniform, but timedependent, Eq. (81) becomes

$$
j^{i}=-\frac{\partial_{t} P_{3}}{2 \pi} \epsilon^{i j k} \partial_{j} A_{k}, i, j, k=x, y, z .
$$

In other words, we have

$$
\vec{j}=-\frac{\partial_{t} P_{3}}{2 \pi} \vec{B}
$$

Since the charge polarization $\vec{P}$ satisfies $\vec{j}=\partial_{t} \vec{P}$, in a static uniform magnetic field $B$ we have $\partial_{t} \vec{P}=$ $-\partial_{t}\left(P_{3} \vec{B} / 2 \pi\right)$, so that

$$
\vec{P}=-\frac{\vec{B}}{2 \pi}\left(P_{3}+\text { const. }\right) .
$$

Such an equation describes the charge polarization induced by a magnetic field, which is a magneto-electric effect. Compared to similar effects in multiferroic materials ${ }^{59,60}$, the magneto-electric effect obtained here is of topological origin, and only determined by the magneto-electric polarization $P_{3}$.

Similar to the $(1+1)$-d adiabatic pumping effect, the response Eq. (84) can be understood in a surface state picture. For example, consider the lattice Dirac model (73) with periodic boundary conditions in the $x, y$ directions and open boundary conditions in the $z$-direction. In the presence of a static magnetic field $B_{z}$ in the $z$ direction, the single particle energy spectrum $E_{n}(\theta)$ can be solved for at a fixed $\theta$ value. As shown in Fig. 11 (b), mid-gap states appear for generic $\theta$, which are localized on the $(2+1)$-d boundary. It should be noticed that each state is $N$-fold degenerate where $N=B_{z} L_{x} L_{y} / 2 \pi$ is the Landau level degeneracy. In the lattice Dirac model, when $-4 c<m<-2 c$ so that $C_{2}=\int_{\theta=0}^{\theta=2 \pi} d P_{3}=1$, we find that during a period $\theta=0 \rightarrow 2 \pi, N$ degenerate surface states on the bottom boundary sink below fermi level and $N$ states on the top float up. Consequently, when $\theta$ is adiabatically tuned from 0 to $2 \pi$, there are $N$ electrons pumped from the top surface to the bottom one, which is in consistent with the result of Eq. (84):

$$
\Delta Q=\int d t \int d x d y j_{z}=-\frac{\int_{0}^{2 \pi} d P_{3}}{2 \pi} B_{z} L_{x} L_{y}=-N C_{2} .
$$

Just like the relation between $(2+1)$-d QH edge states and the mid-gap end states in the $(1+1)$-d pumping effect, there is a direct relationship between the $(3+1)$-d surface states of the $(4+1)$-d lattice Dirac model and the adiabatic pumping discussed above. As discussed in Sec. IIIB the surface theory of a $(4+1)$-d lattice Dirac model with nontrivial $C_{2}$ is a $(3+1)$-d chiral fermion. As shown in Fig. 10 the energy spectrum in a magnetic field $B_{z}$ has a chiral dependence on the wavevector $p_{z}$. During the dimensional reduction procedure, $p_{z}$ (in the notation of Sec. IIIB) is replaced by the parameter $\theta$, so that the chiral energy spectrum $E\left(p_{z}\right)$ changes to the "chiral" $\theta$ dependence of $E_{n}(\theta)$ in Fig. 11 (b). In other words, the adiabatic pumping in the magnetic field in the $(3+1)$-d system is a dimensionally reduced version of the chiral anomaly on the surface of a $(4+1)$-d topological insulator.

The TME leads to a striking consequence if magnetic monopoles are present. For a uniform $P_{3}$, Eq. (84) leads to

$$
\nabla \cdot \vec{j}=-\frac{\partial_{t} P_{3}}{2 \pi} \nabla \cdot \vec{B} .
$$

Suppose we consider a compact $U(1)$ electromagnetic field on a lattice, where the monopole density $\rho_{m}=$ $\nabla \cdot \vec{B} / 2 \pi$ can be non-vanishing, then we obtain

$$
\partial_{t} \rho_{e}=\left(\partial_{t} P_{3}\right) \rho_{m}
$$

Therefore, when $P_{3}$ is adiabatically changed from zero to $\Theta / 2 \pi$, the magnetic monopole will acquire a charge of

$$
Q_{e}=\frac{\Theta}{2 \pi} Q_{m} .
$$

Such a relation was first derived by Witten in the context of the topological term obtained from $\mathrm{QCD}^{61}$.

\section{C. $Z_{2}$ topological classification of time-reversal invariant insulators}

In Sec. IID we have seen how a $Z_{2}$ topological classification is obtained for $(1+1)$-d particle-hole symmetric insulators. The key point for that case is to show 
that any interpolation between two particle-hole symmetric insulators $h_{1}(k)$ and $h_{2}(k)$ carries the same parity of Chern number, so that the "relative Chern parity" is well-defined for each two Hamiltonians with particle-hole symmetry. In this section, we will show that the same approach can be applied to $(3+1)$-d insulators, where the time-reversal symmetry plays the same role as particlehole symmetry does in $(1+1)$-d.

For a Hamiltonian $H=\sum_{m, n} c_{m \alpha}^{\dagger} h_{m n}^{\alpha \beta} c_{n \beta}$, the timereversal transformation is an anti-unitary operation defined by $c_{m \alpha} \rightarrow T^{\alpha \beta} c_{m \beta}$, where the time-reversal matrix $T$ satisfies $T^{\dagger} T=\mathbb{I}$ and $T^{*} T=-\mathbb{I}$. In $\vec{k}$-space timereversal symmetry requires

$$
T^{\dagger} h(-\vec{k}) T=h^{T}(\vec{k}) .
$$

The condition $T^{*} T=-\mathbb{I}$ is essential, and leads to Kramers's degeneracy. Now we will follow the same approach as Sec. IID and show how to define a $Z_{2}$ invariant for the TRI insulators in $(3+1)$-d. For any two TRI band insulators $h_{1}(\vec{k})$ and $h_{2}(\vec{k})$, an interpolation $h(\vec{k}, \theta)$ can be defined, satisfying

$$
\begin{array}{r}
h(\vec{k}, 0)=h_{1}(\vec{k}), h(\vec{k}, \pi)=h_{2}(\vec{k}) \\
T^{\dagger} h(-\vec{k},-\theta) T=h^{T}(\vec{k}, \theta),
\end{array}
$$

and $h(\vec{k}, \theta)$ is gapped for any $\theta \in[0,2 \pi]$. Since the interpolation is periodic in $\theta$, a second Chern number $C_{2}[h(\vec{k}, \theta)]$ of the Berry phase gauge field can be defined in the $(\vec{k}, \theta)$ space. In the same way as in Sec. IID we will demonstrate below that $C_{2}[h(\vec{k}, \theta)]-C_{2}\left[h^{\prime}(\vec{k}, \theta)\right]=$
$0 \bmod 2$ for any two interpolations $h$ and $h^{\prime}$. First of all, two new interpolations $g_{1,2}(\vec{k}, \theta)$ can be defined by Eq. (39), which we repeat here for convenience:

$$
\begin{aligned}
& g_{1}(k, \theta)=\left\{\begin{array}{cc}
h(k, \theta), & \theta \in[0, \pi] \\
h^{\prime}(k, 2 \pi-\theta), & \theta \in[\pi, 2 \pi]
\end{array}\right. \\
& g_{2}(k, \theta)=\left\{\begin{array}{cc}
h^{\prime}(k, 2 \pi-\theta), & \theta \in[0, \pi] \\
h(k, \theta), & \theta \in[\pi, 2 \pi]
\end{array} .\right.
\end{aligned}
$$

By their definition, $g_{1}$ and $g_{2}$ satisfy $C_{2}[h]-C_{2}\left[h^{\prime}\right]=$ $C_{2}\left[g_{1}\right]+C_{2}\left[g_{2}\right]$ and $T^{\dagger} g_{1}(-\vec{k},-\theta) T=g_{2}^{T}(\vec{k}, \theta)$. To demonstrate $C_{2}\left[g_{1}\right]=C_{2}\left[g_{2}\right]$, consider an eigenstate $|\vec{k}, \theta ; \alpha\rangle_{1}$ of $g_{1}(\vec{k}, \theta)$ with eigenvalue $E_{\alpha}(\vec{k}, \theta)$. We have

$$
\begin{aligned}
g_{2}^{T}(-\vec{k},-\theta) T^{\dagger}|\vec{k}, \theta ; \alpha\rangle_{1} & =T^{\dagger} g_{1}(\vec{k}, \theta)|\vec{k}, \theta ; \alpha\rangle_{1} \\
& =E_{\alpha}(\vec{k}, \theta) T^{\dagger}|\vec{k}, \theta ; \alpha\rangle_{1} \\
\Rightarrow g_{2}(-\vec{k},-\theta) T^{T}\left(|\vec{k}, \theta ; \alpha\rangle_{1}\right)^{*} & =E_{\alpha}(\vec{k}, \theta) T^{T}\left(|\vec{k}, \theta ; \alpha\rangle_{1}\right)^{*} .
\end{aligned}
$$

Thus $T^{T}\left(|\vec{k}, \theta ; \alpha\rangle_{1}\right)^{*}$ is an eigenstate of $g_{2}(-\vec{k},-\theta)$ with the same eigenvalue $E_{\alpha}(\vec{k}, \theta)$. Expand over the eigenstates $|-\vec{k},-\theta, \beta\rangle_{2}$ of $g_{2}(-\vec{k},-\theta)$, we have

$$
T^{T}\left(|\vec{k}, \theta ; \alpha\rangle_{1}\right)^{*}=\sum_{\beta} U_{\alpha \beta}(\vec{k}, \theta)|-\vec{k},-\theta ; \beta\rangle_{2} .
$$

Consequently the Berry phase gauge vector of the $g_{1}$ and $g_{2}$ systems satisfies

$$
\begin{aligned}
a_{1 j}^{\alpha \beta}(\vec{k}, \theta) & =-i\left\langle\vec{k}, \theta ; \alpha\left|\partial_{j}\right| \vec{k}, \theta ; \beta\right\rangle_{1}=-i\left[\sum_{\gamma, \delta} U_{\alpha \gamma}^{*}\langle-\vec{k},-\theta ; \gamma| \partial_{j}\left(U_{\beta \delta}|-\vec{k},-\theta ; \delta\rangle_{2}\right)\right]^{*} \\
& =\sum_{\gamma, \delta} U_{\alpha \gamma} a_{2 j}^{\gamma \delta *}(-\vec{k},-\theta)\left(U^{\dagger}\right)_{\delta \beta}-i \sum_{\gamma} U_{\alpha \gamma}(\vec{k}, \theta) \partial_{j} U_{\beta \gamma}^{*}(\vec{k}, \theta) .
\end{aligned}
$$

In other words, $a_{1 j}^{\alpha \beta}(\vec{k}, \theta)$ is equal to $a_{2 j}^{\alpha \beta}(-\vec{k},-\theta)$ up to a gauge transformation. Consequently, the Berry phase curvature satisfies $f_{1 i j}^{\alpha \beta}(\vec{k}, \theta)=U_{\alpha \gamma} f_{2 i j}^{\gamma \delta *}(-\vec{k},-\theta)\left(U^{\dagger}\right)_{\delta \beta}$, which thus leads to $C_{2}\left[g_{1}(\vec{k}, \theta)\right]=C_{2}\left[g_{2}(\vec{k}, \theta)\right]$. In summary, we have proved $C_{2}[h(\vec{k}, \theta)]-C_{2}\left[h^{\prime}(\vec{k}, \theta)\right]=$ $2 C_{2}[g(\vec{k}, \theta)]=0 \bmod 2$ for any two symmetric interpolations $h$ and $h^{\prime}$. Thus the "relative second Chern parity"

$$
N_{3}\left[h_{1}(\vec{k}), h_{2}(\vec{k})\right]=(-1)^{C_{2}[h(\vec{k}, \theta)]}
$$

is well-defined for any two time-reversal invariant $(3+1)$ $\mathrm{d}$ insulators, independent on the choice of interpolation. In the same way as in $(1+1)$-d, a vacuum Hamiltonian $h_{0}(\vec{k}) \equiv h_{0}, \forall \vec{k}$ can be defined as a reference. All the
Hamiltonians with $N_{3}\left[h_{0}, h\right]=-1$ are called $Z_{2}$ nontrivial, while those with $N_{3}\left[h_{0}, h\right]=1$ are trivial.

Similar to the $(1+1)$-d case, there is a more intuitive, but less rigorous, way to define the $Z_{2}$ invariant $N_{3}$. Through the derivation of Eq. (91) one can see that for a TRI Hamiltonian satisfying Eq. (88), the Berry's phase gauge potential satisfies $a_{i}(\vec{k})=U a_{i}(-\vec{k}) U^{\dagger}-i U \partial_{i} U^{\dagger}$, so that the magneto-electric polarization $P_{3}$ satisfies

$$
2 P_{3}=\frac{i}{24 \pi^{2}} \int d^{3} k \epsilon^{i j k} \operatorname{Tr}\left[\left(U \partial_{i} U^{\dagger}\right)\left(U \partial_{j} U^{\dagger}\right)\left(U \partial_{k} U^{\dagger}\right)\right] \in \mathbb{Z} .
$$

Consequently, there are only two inequivalent, TRI values of $P_{3}$, which are $P_{3}=0$ and $P_{3}=1 / 2$. For two Hamiltonians $h_{1}$ and $h_{2}$, the second Chern number 
$C_{2}[h(\vec{k}, \theta)]=2\left(P_{3}\left[h_{2}\right]-P_{3}\left[h_{1}\right]\right) \bmod 2$, so the difference of $P_{3}$ determines the relative Chern parity $N_{3}\left[h_{1}, h_{2}\right]$ by $N_{3}\left[h_{1}, h_{2}\right]=(-1)^{2\left(P_{3}\left[h_{1}\right]-P_{3}\left[h_{2}\right]\right)}$. Since the trivial Hamiltonian $h_{0}$ obviously has $P_{3}=0$, we know that all the Hamiltonians with $P_{3}=1 / 2$ are topologically non-trivial, while those with $P_{3}=0$ are trivial.

Once the $Z_{2}$ classification is obtained, the physical consequences of this topological quantum number can be studied by the effective theory (80), as has been done in the last subsection. In the $(1+1)$-d case, we have shown that a zero-energy localized state exists at each open boundary of a $Z_{2}$ nontrivial particle-hole symmetric insulator, which leads to a half charge $Q_{d}=e / 2(\bmod e)$ confined on the boundary. Similarly, the nontrivial $(3+1)$ $\mathrm{d}$ insulators also have topologically protected surface states. The easiest way to study the surface physics of the $(3+1)$-d insulator is again by dimensional reduction. As discussed above, for any three-dimensional Hamiltonian $h_{1}(\vec{k})$, an interpolation $h(\vec{k}, \theta)$ can be defined between $h_{1}$ and the "vacuum Hamiltonian" $h_{0}$. If we interpret $\theta$ as the fourth momentum, $h(\vec{k}, \theta)$ defines a $(4+1)$ $\mathrm{d}$ band insulator. Moreover, the constraint Eq. (89) on $h(\vec{k}, \theta)$ requires time-reversal symmetry for the corresponding $(4+1)$-d system. The Hamiltonian $h(\vec{k}, \theta)$ can be written in a real space form and then defined on a fourdimensional lattice with open boundary conditions in the $z$-direction and periodic boundary conditions for all the other directions. As discussed in Sec. IIIB there will be $\left|C_{2}[h]\right|$ flavors of $(3+1)$-d chiral fermions on the surface when the second Chern number $C_{2}[h]$ is nonzero. In other words, in the $3 \mathrm{D} \mathrm{BZ}$ of the surface states there are $\left|C_{2}[h]\right|$ nodal points $\left(k_{x n}, k_{y n}, \theta_{n}\right), n=1, . .,\left|C_{2}[h]\right|$ where the energy spectrum $E_{n}\left(k_{x}, k_{y}, \theta\right)$ is gapless and disperses linearly as a Dirac cone. From time-reversal symmetry it is easy to prove that the energy spectrum is identical for $\left(k_{x}, k_{y}, \theta\right)$ and $\left(-k_{x},-k_{y},-\theta\right)$. Consequently, if $\left(k_{x}, k_{y}, \theta\right)$ is a nodal point, so is $\left(-k_{x},-k_{y},-\theta\right)$. In other words, time-reversal symmetry requires the chiral fermions to appear in pairs, except for the ones at timereversal symmetric points, as shown in Fig. 12 (a). Thus, when the second Chern number $C_{2}[h]$ is odd, there must be an odd number of Dirac cones at the 8 symmetric points in the 3D BZ. Actually, the $(4+1)$-d lattice Dirac model (62) provides an example of TRI insulators with nontrivial second Chern number, since one can define $\Gamma^{0}$ to be time-reversal even and $\Gamma^{1,2,3,4}$ to be odd, as in conventional relativistic quantum mechanics $\frac{62}{2}$. As shown in Fig. 9, all the nodal points of the surface states are located at the symmetric points $\Gamma, M, R$ or $X$.

Now we return to the surface of $(3+1)$-d insulator. Since $h_{1}(\vec{k})=h(\vec{k}, 0), h_{0}=h(\vec{k}, \pi)$ by definition of the interpolation, the surface energy spectra of $h_{1}$ and $h_{0}$ are given by the $\theta=0$ and $\theta=\pi$ slices of the 3D surface spectrum. Since all 8 time-reversal symmetric points $(\Gamma$, $X$ and $M$ ) are at $\theta=0$ or $\theta=\pi$, we know that the net number of Dirac cones on the surface energy spectrum of $h_{1}$ and $h_{0}$ is odd (even) when $C_{2}[h(\vec{k}, \theta)]$ is odd (even). (a)

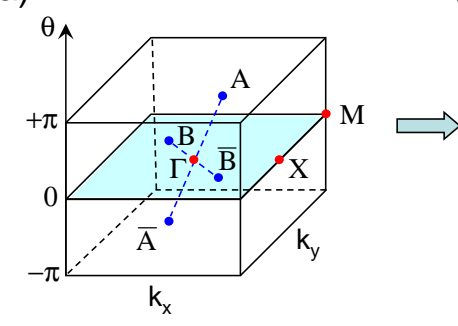

(b)

$(3+1) d$ surface of
$(4+1) d$ system

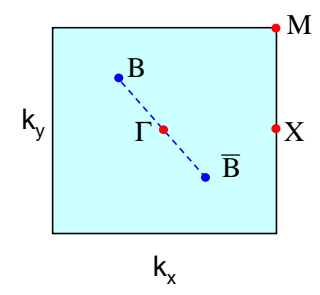

$(2+1)$-d surface of

$(3+1) d$ system

FIG. 12: Illustration of the nodal points in the surface state energy spectrum of $(4+1)$-d and $(3+1)$-d insulators. (a) The nodal points in the $\left(k_{x}, k_{y}, \theta\right) \mathrm{BZ}$, for a $z=$ const. surface of the $(4+1)$-d system. The red (blue) points stand for nodal points at time-reversal symmetric (asymmetric) wavevectors. The dashed lines are a guide for the eyes. There are two pairs of asymmetric nodal points and three symmetric points in this example, which correspond to a bulk second Chern number $C_{2}=7$. (b) The nodal points in the $\left(k_{x}, k_{y}\right) \mathrm{BZ}$, for a $z=$ const. surface of the $(3+1)$-d system. According to the dimensional reduction procedure (see text) the $2 \mathrm{D}$ surface energy spectrum is given by the $\theta=0$ slice of the $3 \mathrm{D}$ surface spectrum in (a). Since $\theta=\pi$ corresponds to a vacuum Hamiltonian $h_{0}$, no nodal points exist in that plane. Consequently, the number of nodal points in the $2 \mathrm{D} \mathrm{BZ} \mathrm{(5} \mathrm{in} \mathrm{this} \mathrm{example)}$ has the same parity as $C_{2}$ in the $(4+1)$-d system.

However, $h_{0}$ is defined as the vacuum Hamiltonian, which is totally local without any hopping between different sites. Thus, there cannot be any mid-gap surface states for $h_{0}$. Consequently, the number of 2D Dirac cones in the surface state spectrum of $h_{1}$ must be odd (even) when $C_{2}[h]$ is odd (even). Since the parity of $C_{2}[h]$ determines the $Z_{2}$ invariant $N_{3}\left[h_{1}\right]$, we finally reach the conclusion that there must be an odd (even) number of $(2+1)$ $d$ gapless Dirac fermions confined on the surface of a $(3+1)$-d nontrivial (trivial) topological insulator.

Compared to earlier works on $Z_{2}$ invariants and surface states in $(3+1)$-d, one can see that the $Z_{2}$ nontrivial topological insulator defined here corresponds to the "strong topological insulator" of Ref. 22. The present approach has the advantage of (i) demonstrating the bulk-edge relationship more explicitly, (ii) clarifying the connection between the second Chern number and the $Z_{2}$ topological number and (iii) naturally providing the effective theory that describes the physically measurable topological response properties of the system. The "weak topological insulators" defined in Ref. 22 are not included in the present approach, since these $Z_{2}$ invariants actually correspond to topological properties of $(2+1)$-d insulators (QSH insulators, as will be discussed in next section), just as the QH effect in $(3+1)$-d systems $\underline{63}$ still corresponds to a first Chern number, but defined in a $2 \mathrm{D}$ projection of the 3D BZ. 


\section{Physical properties of $Z_{2}$-nontrivial insulators}

In the last subsection we have defined the $Z_{2}$ topological quantum number for the $(3+1)$-d TRI insulators, and discussed the gapless Dirac fermions on the surface of a non-trivial insulator. Now we will study the physical response properties of the non-trivial insulators. Since the non-trivial insulator has a magneto-electric polarization $P_{3}=1 / 2 \bmod 1$, according to Eq. (80) the effective action of the bulk system should be

$$
S_{3 \mathrm{D}}=\frac{2 n+1}{8 \pi} \int d^{3} x d t \epsilon^{\mu \nu \sigma \tau} \partial_{\mu} A_{\nu} \partial_{\sigma} A_{\tau} .
$$

in which $n=P_{3}-1 / 2 \in \mathbb{Z}$ is the integer part of $P_{3}$. Under time-reversal symmetry, the term $\epsilon^{\mu \nu \sigma \tau} \partial_{\mu} A_{\nu} \partial_{\sigma} A_{\tau}=$ $2 \mathbf{E} \cdot \mathbf{B}$ is odd, so that for general $P_{3}$, the effective action (80) breaks time-reversal symmetry. However, when the space-time manifold is closed (i.e., with periodic boundary conditions in the spatial and temporal dimensions), the term $\int d^{3} x d t \epsilon^{\mu \nu \sigma \tau} \partial_{\mu} A_{\nu} \partial_{\sigma} A_{\tau}$ is quantized to be $8 \pi^{2} m, m \in \mathbb{Z}$. Consequently, $S_{3 \mathrm{D}}=(2 n+1) m \pi$ so that the action $e^{i S_{3 \mathrm{D}}}=e^{i m \pi}=(-1)^{m}$ is time-reversal invariant and is independent of $n$, the integer part of $P_{3}$. This time-reversal property of the effective action is consistent with that of $P_{3}$ discussed in the last subsection. Thus, in a closed space-time manifold, the topological action (92) is a consistent effective theory of the $Z_{2}$-nontrivial TRI insulators, and the integer part of $P_{3}$ is not a physical quantity. However, the case is different when the system has open boundaries. On a space-time manifold with boundary, the value of $S_{3 \mathrm{D}}$ is not quantized, which thus breaks time-reversal symmetry even for $P_{3}=1 / 2$ or 0 . In this case, the integer part $n$ of $P_{3}$ does enter the action $e^{i S_{3 \mathrm{D}}}$, and becomes physical; its value depends on the quantitative details of the boundary.

To understand the physics in the open boundary system, we study a semi-infinite nontrivial insulator occupying the space $z \leq 0$. Since the vacuum, which fills $z>0$, is effectively a trivial insulator (with an infinitely large gap), the effective action (80) can be written in the whole of $\mathbb{R}^{3}$ as

$$
S_{3 \mathrm{D}}=\frac{1}{4 \pi} \int d^{3} x d t \epsilon^{\mu \nu \sigma \tau} A_{\mu} \partial_{\nu} P_{3} \partial_{\sigma} A_{\tau}
$$

Since $P_{3}=1 / 2 \bmod 1$ for $z<0$ and $P_{3}=0 \bmod 1$ for $z>0$, we have

$$
\partial_{z} P_{3}=(n+1 / 2) \delta(z),
$$

where $n \in \mathbb{Z}$ depends on the non-topological details of the surface, as will be studied later. In this case, the effective action is reduced to a $(2+1)$-d Chern-Simons term on the surface:

$$
S_{\text {surf }}=-\frac{2 n+1}{8 \pi} \int d x d y d t \epsilon^{3 \mu \nu \rho} A_{\mu} \partial_{\nu} A_{\rho} .
$$

This is consistent with the observation in Sec. IVB that a domain wall of $P_{3}$ carries a $\mathrm{QH}$ effect. The Hall conductance of such a surface of a $Z_{2}$ nontrivial insulator is thus $\sigma_{H}=(n+1 / 2) / 2 \pi$, which is quantized as a half odd integer times the quanta $e^{2} / h$. On the other hand, from the discussion in the last subsection we know that there are always an odd number of $(2+1)$-d Dirac fermions living on the surface of a nontrivial insulator. Thus the half QH effect on the surface can be easily understood by the parity anomaly of massless Dirac fermions ${ }^{64}$. Here we need to be more careful. The Hall conductance carried by a Dirac fermion is well-defined only when the fermion mass is non-vanishing, so that a gap is opened. With the continuum Hamiltonian $H=k_{x} \sigma^{x}+k_{y} \sigma^{y}+m \sigma^{z}$, the Berry phase curvature can be calculated as in Eq. (11). The $\mathbf{d}$ vector is given by $\mathbf{d}=\left(k_{x}, k_{y}, m\right)$, which has a meron-type configuration in $k_{x}, k_{y}$ space, and thus carries a Hall conductance ${ }^{64}$

$$
\sigma_{H}=\frac{1}{4 \pi} \operatorname{sgn}(m)\left(=\frac{e^{2}}{2 h} \operatorname{sgn}(m)\right)
$$

Now consider the surface of a topological insulator with $2 n+1$ gapless Dirac cones. From the discussion in the last subsection we know that without breaking time-reversal symmetry, at least one of these Dirac cones cannot be gapped. Now consider a perturbation that breaks timereversal symmetry, i.e. a term which assigns a mass $m_{i}, i=1,2, . .2 n+1$ to each Dirac cone and induces a net Hall conductance $\sigma_{H}=\sum_{i=1}^{2 n+1} \operatorname{sgn}\left(m_{i}\right) / 4 \pi$. Since $\sum_{i=1}^{2 n+1} \operatorname{sgn}\left(m_{i}\right)$ is an odd integer, the Hall conductance we obtain is consistent with the surface Chern-Simons theory (93). From this discussion we can understand that the effective action (93) describes a surface with time-reversal symmetry breaking, though the bulk system remains time-reversal invariant. The bulk topology requires there to be a $1 / 2$ quanta in the Hall conductance, and the surface time-reversal symmetry breaking term determines the integer part $n$. This is an exact analog of the half charge on an end of the $(1+1)$-d particlehole symmetric insulator. As shown in Fig. 5, whether the localized state on the end of a nontrivial insulator is filled or vacant can only be determined by choosing a chemical potential $\mu_{1}>0$ or $\mu_{2}<0$, or equivalently, by breaking the particle-hole symmetry around the boundary. The charge localized at one end of the insulator chain is $(n+1 / 2) e$, in which the integer part $n$ depends on the symmetry breaking term on the surface, but the $1 / 2$ part is guaranteed by the bulk topology.

To show such a surface state picture more explicitly, again the lattice Dirac model can be taken as an example. Consider the $(3+1)$-d lattice Dirac model (73) with a domain wall configuration of the $\theta(\vec{x})$ field given by

$$
\theta(\vec{x})=\theta(z)=\frac{\pi}{2}[1-\tanh (z / 4 \xi)]
$$

which has the asymptotic behavior $\theta(z \rightarrow-\infty)=\pi$, $\theta(z \rightarrow+\infty)=0$. The domain wall width is $\xi$, as shown in Fig. 13 (a). With periodic boundary conditions in the $x$ and $y$-directions, the Hamiltonian can be block 
(a)
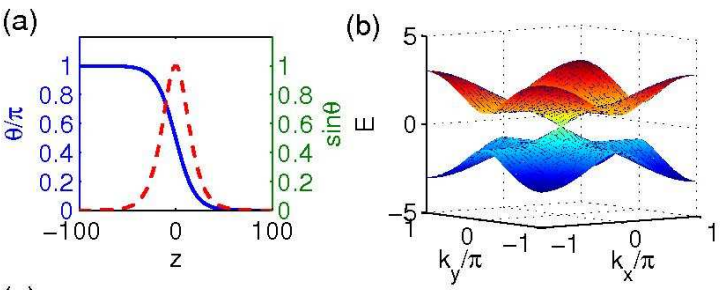

(c)

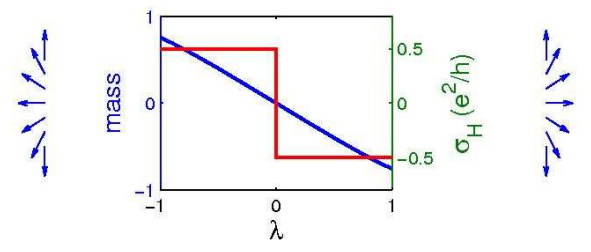

FIG. 13: (a) Domain wall configuration $\theta(z)$ (blue line) and corresponding $\sin \theta(z)$ (red dashed line) as defined by Eq. (94) with $\xi=20$. (b) Dispersion relation of the two-dimensional bands trapped on the domain wall for the TRI Hamiltonian $h_{0}$. (c) The mass of surface Dirac fermion (blue line) and the half-quantized Hall conductance carried by the domain wall (red line) in the Hamiltonian $h(\lambda)=h_{0}+\lambda h_{1}$ as a function of $\lambda$. The arrows on the left and right side show schematically the rotation of angle $\theta$ across the domain wall for $\lambda<0$ and $\lambda>0$, respectively.

diagonalized:

$$
\begin{aligned}
H & =\sum_{z, k_{x}, k_{y}}\left[\psi_{k_{x} k_{y}}^{\dagger}(z)\left(\frac{c \Gamma^{0}-i \Gamma^{3}}{2}\right) \psi_{k_{x} k_{y}}(z+1)+\text { h.c. }\right] \\
& +\sum_{z, k_{x}, k_{y}} \psi_{k_{x} k_{y}}^{\dagger}(z)\left[\left(m+c \cos \theta(z)+c \cos k_{x}\right.\right. \\
& \left.\left.+c \cos k_{y}\right) \Gamma^{0}+\sin k_{x} \Gamma^{1}+\sin k_{y} \Gamma^{2}\right] \psi_{k_{x} k_{y}}(z) \\
& +\sum_{z, k_{x}, k_{y}} \psi_{k_{x} k_{y}}^{\dagger}(z) \sin \theta(z) \Gamma^{4} \psi_{k_{x} k_{y}}(z) \equiv H_{0}+H_{1} .(95)
\end{aligned}
$$

Under a time-reversal transformation, $\Gamma^{0}$ is even and $\Gamma^{1,2,3,4}$ are odd. Thus the only time-reversal odd term in Hamiltonian (95) is the last term, which is localized around the boundary due to the factor $\sin \theta(z)$ (See Fig. (13) (a)). Hereby we denote $H=H_{0}+H_{1}$ with $H_{1}$ the last term and $H_{0}$ all the other TRI terms, and define $h_{0}, h_{1}$ as the single particle Hamiltonian corresponding to $H_{0}, H_{1}$, respectively. Then the Hamiltonian $H_{0}$ describes a time-reversal invariant interface between two insulators $\theta=0$ and $\theta=\pi$. For $-4 c<m<-2 c$, the parameterized Hamiltonian $H(\theta), \theta \in[0,2 \pi]$ has a Chern number $C_{2}=1$, so that the $\theta=0$ and $\theta=\pi$ system has relative Chern parity -1 . It's easy to show that Hamiltonian (73) for $\theta=0,-4 c<m<-2 c$ is adiabatically connected to the $m \rightarrow-\infty$ limit. Thus we know that $\theta=0$ and $\theta=\pi$ correspond to $Z_{2}$ trivial and nontrivial insulators, respectively. Consequently, on the domain wall at $z=0$ there should be an odd number of gapless Dirac cones for $H_{0}$. As shown in Fig. 13 (b), numerical diagonalization of $h_{0}$ shows one single Dirac cone at $\left(k_{x}, k_{y}\right)=(0,0)$ on the surface. To understand the ef- fect of $h_{1}$ term, notice that $\left\{\Gamma^{4}, h_{0}\right\}=0$, with \{\} being the anti-commutator. The effective Hamiltonian of the Dirac cone can always be written as $h_{\text {surf }}=k_{x} \sigma_{x}+k_{y} \sigma_{y}$ in a proper basis, and it should also anti-commute with $\Gamma^{4}$ since the bulk Hamiltonian does. Since the only term that anti-commutes with $h_{\text {surf }}$ in the $2 \times 2$ Hilbert space is $\sigma_{z}$, we know that the effect of $\Gamma^{4}$ term is to induce a mass term $m \sigma_{z}$ in the lattice Dirac model. More accurately, the amplitude and the sign of $m$ can be determined by standard perturbation theory. Given the two zero-energy surface states $|k=0, \alpha\rangle, \alpha=1,2$, the representation of the matrices $\sigma_{x}$ and $\sigma_{y}$ in the effective theory $h_{\text {surf }}$ can be determined by

$$
\sigma_{\alpha \beta}^{i}=\left\langle k=0, \alpha\left|\frac{\partial h_{0}}{\partial k_{i}}\right|_{k=0} \mid k=0, \beta\right\rangle, i=x, y .
$$

Then the $\sigma^{z}$ is given by $\sigma^{z}=-i \sigma^{x} \sigma^{y}$, so that the mass $m$ is determined by

$$
m=\frac{1}{2} \sum_{\alpha \beta} \sigma_{\alpha \beta}^{z}\left\langle k=0, \beta\left|h_{1}\right| k=0, \alpha\right\rangle .
$$

If we consider the parameterized Hamiltonian $h=h_{0}+$ $\lambda h_{1}$, then the mass of the surface Dirac fermion is proportional to $\lambda$ for $\lambda \rightarrow 0$. As shown in Fig. 13 (c), the mass is positive for $\lambda<0$, which leads to a surface Hall conductance $\sigma_{H}=-\operatorname{sgn}(\lambda) / 4 \pi$. On the other hand, the surface Hall conductance can also be calculated by the effective theory through Eq. (82). For $\lambda=1$, the phase field $\theta$ winds from $\pi$ to 0 , which leads to $\sigma_{H}=\int_{-\infty}^{+\infty} d P_{3}(z) / 2 \pi=\int_{\pi}^{0} d P_{3}(\theta) / 2 \pi=-1 / 4 \pi$ (since $\left.\int_{0}^{\pi} d P_{3}=C_{2} / 2=1 / 2\right)$. The Hamiltonian for $\lambda=-1$ can be considered to be the same lattice Dirac Hamiltonian $H(\theta)$ with $\theta(z)$ replaced by $-\theta(z)$. This keeps $h_{0}$ invariant but reverses the sign of $h_{1}$. Consequently, the winding of the $\theta$ field is from $-\pi$ to 0 , which leads to a Hall conductance $\sigma_{H}=\int_{-\pi}^{0} d P_{3}(\theta) / 2=1 / 4 \pi$. The winding of $\theta$ in the two cases is shown schematically in Fig. 13 (c).

In summary, from this example we learn that the effect of a time-reversal symmetry breaking term on the surface is to assign a mass to the Dirac fermions which determines the winding direction of $P_{3}$ through the domain wall. Once each Dirac cone on the surface gains a mass, the whole system is gapped and the Berry phase curvature is well-defined, so that the winding number of $P_{3}$ through the domain wall is determined. Physically, the time-reversal symmetry breaking term on the surface can come from magnetic fields or magnetic moments localized on the surface; it could also arise from the spontaneous breaking of time reversal symmetry on the surface due to interactions. Once such a "T-breaking surface field" (denoted by $M$ ) is applied, the effective action (80) is well-defined for open boundaries, and describes the electromagnetic response of the $Z_{2}$ nontrivial insulator. Actually, the T-breaking field should be considered to be an external field applied to the TRI system, such that the 
topological action (80) describes a nonlinear response of the system to the combination of $M$ and electromagnetic field $A_{\mu}$. For a $Z_{2}$ nontrivial insulator occupying a spatial region $\mathcal{V}$ with boundary $\partial \mathcal{V}$, the spatial gradient of $P_{3}$ is given by

$$
\nabla P_{3}(\vec{x})=\left(g[M(\vec{x})]+\frac{1}{2}\right) \int_{\partial \mathcal{V}} d \hat{\mathbf{n}}(\vec{y}) \delta^{3}(\vec{x}-\vec{y})
$$

where $g[M(\vec{x})] \in \mathbb{Z}$ is the integer part of the winding number determined by the T-breaking field $M(\vec{x})$, and $\hat{\mathbf{n}}$ is the normal vector of the surface. Under such a configuration of $\nabla P_{3}$, the effective action (80) is reduced to the surface Chern-Simons action

$$
S_{\text {surf }}=\frac{1}{4 \pi} \int_{\partial \mathcal{V}} d \hat{n}_{\mu}\left(g[M(\vec{x})]+\frac{1}{2}\right) \epsilon^{\mu \nu \sigma \tau} A_{\nu} \partial_{\sigma} A_{\tau}
$$

Since $g[M(\vec{x})]$ can only take discrete values, in general the surface of a nontrivial insulator consists of several domains with different Hall conductance. To obtain more realistic predictions of the effective theory (97), in the rest of this subsection we will study a specific case - the interface between a ferromagnetic insulator and a $Z_{2}$ nontrivial insulator, where the surface time-reversal symmetry breaking is generated by the magnetization of the FM material. Several specific experimental proposals will be discussed.

\section{(1) Magnetization-induced QH effect.}

Consider the ferromagnet-topological insulator heterostructure shown in Fig. 14. The magnetization of the two FM layers can be parallel or antiparallel, and the standard six-terminal measurement can be performed to measure the in-plane Hall conductance. The net Hall conductance is given by the summation of the contributions of the top and bottom surfaces. When the topological insulator is uniform, an outward pointing magnetization vector, (i.e., towards the direction of the surface normal vector $\hat{\mathbf{n}}$ ), will have the same effect, no matter to which surface it is applied. Suppose the Hall current on the top surface induced by an electric field $\mathbf{E}=E_{x} \hat{\mathbf{x}}$ is $\mathbf{j}_{t}=\hat{\mathbf{n}}_{t} \times \mathbf{E} / 4 \pi$, then on the bottom surface the same formula applies, such that $\mathbf{j}_{b}=\hat{\mathbf{n}}_{b} \times \mathbf{E} / 4 \pi$. Since $\hat{\mathbf{n}}_{t}=-\hat{\mathbf{n}}_{b}=\hat{\mathbf{z}}$, the current $\mathbf{j}_{t}=-\mathbf{j} b$, as shown in Fig. 14. Consequently, the antiparallel magnetization leads to a vanishing net Hall conductance, while the parallel magnetization leads to $\sigma_{H}=e^{2} / h$.

Just like the usual integer QH effect, the quantized Hall conductance here is carried by chiral edge states. To understand the edge state picture, notice that for parallel magnetizations in Fig. 14(a), the magnetization vector is outward pointing at the top surface and inward pointing at the bottom surface. Although the Hall conductance of the two surfaces are the same in the global $x, y, z$ basis, they are opposite in the local basis defined with respect to the normal vector $\hat{\mathbf{n}}$. In other words, the integer $g[M(\vec{x})]$ in the surface Chern-Simons theory (97) is 0 for the top surface and -1 for the bottom surface. Consequently, the side surface is a domain wall between two different $\mathrm{QH}$
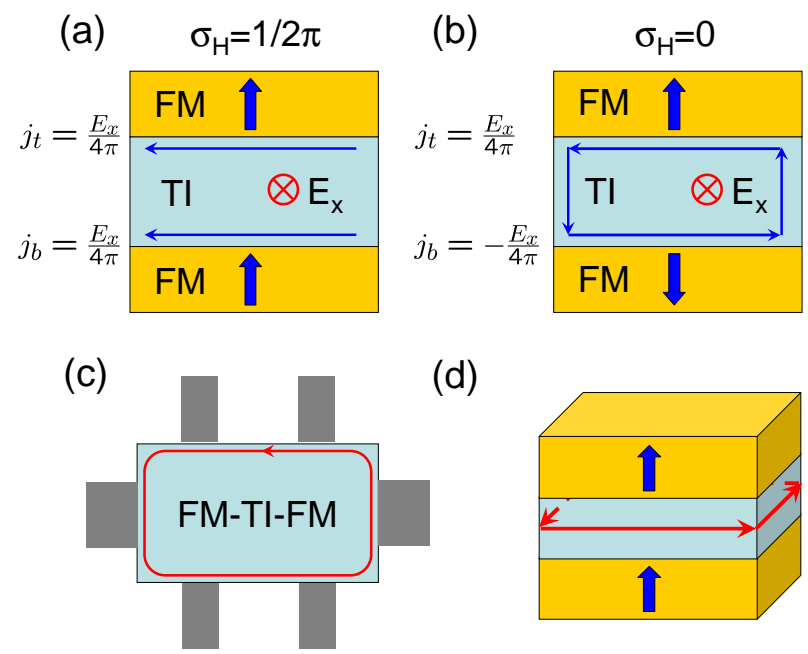

(d)

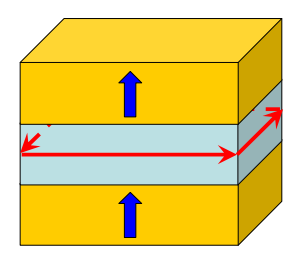

FIG. 14: Illustration of the $\mathrm{QH}$ effect in ferromagnettopological insulator heterostructure. (a) and (b) The electric field $E_{x}$ (with direction into the paper) and the induced Hall current $j_{t}$ and $j_{b}$ for parallel and antiparallel magnetization, respectively. In (b) the Hall current on the two surfaces are opposite and form a circulating current. (c) A top-down view of the device for Hall measurement. The grey regions are leads that contact to the surface of the topological insulator. (d) For the case of parallel magnetization, the chiral edge states are trapped on the side surfaces of the topological insulator. These carry the quantized Hall current.

regions with Hall conductances that differ by one quantum. Just like a domain wall between $\nu=0$ and $\nu=1$ regions in the usual QH system, such a domain wall will trap a chiral fermi liquid, which in this experimental proposal is responsible for the net Hall effect. It should be noticed that the side surface is $2 \mathrm{D}$, so that generically there are also other non-chiral propagating modes on the side surface, besides the one branch of chiral edge states. However, the existence of these non-chiral states does not change the stability of the chiral edge state, since there is always one more right mover than left mover. The stability of the QH effect is still protected by the "bulk" gap, which is the magnetization-induced gap $E_{M}$ in this case. Thus we will expect to observe this QH effect under the following two requirements: (i) temperature $k_{B} T \ll E_{M}$; (ii) the chemical potential on the top and bottom surfaces remains in the gap induced by the applied magnetization.

We would like to point out that this experimental proposal provides a direct demonstration of the half $\mathrm{QH}$ effect on the surface of a topological insulator. If the $\sigma_{H}=e^{2} / h$ measured for parallel magnetization were contributed by one surface, then the magnetization flip of the other surface would have no effect on the net Hall conductance. Thus if an $e^{2} / h$ Hall conductance is observed for parallel magnetization, and the magnetization flip of either magnet leads to vanishing Hall conductance, one can conclude that the Hall conductance is contributed equally by the two surfaces. 
(2) Topological Magneto-Electric Effect (TME).

As has been discussed in Sec. IVB, a TME effect is induced by $P_{3}$, which is described by Eqs. (84) and (85). Now we consider the realization of this effect in a nontrivial topological insulator. Similar to the surface $\mathrm{QH}$ effect, a magnetization (or any other time-reversal symmetry breaking term) is necessary to determine the integer part of $P_{3}$. Consider the FM-TI-FM structure in Fig. 14 (b). With antiparallel magnetizations, the current induced by an electric field $E_{x}$ on the top and bottom surfaces flows in opposite directions. If we consider an isolated system rather than the Hall bar with leads as discussed above, a circulating current is formed, which induces a magnetic field parallel or anti-parallel to the electric field. However, in the geometry shown in Fig. (14) (b), dissipation occurs when the circulating current flows on the gapless side surface and the adiabatic condition of the TME effect is violated. To obtain the TME effect, a T-breaking gap for the side surface is necessary. This is satisfied in the cylindrical geometry shown in Fig. 15 (a). With a magnetization pointing out of the cylinder's surface, the surface is gapped and has a fixed Hall conductance $\sigma_{H}=\left(n+\frac{1}{2}\right) e^{2} / h$. In an electric field parallel to the cylinder as shown in Fig. 15 (a), a tangential circulating current is induced, with the strength $j_{t}=\sigma_{H} E$. The magnetic field generated by such a current in the topological insulator can be obtained by solving Maxwell's equations:

$$
\mathbf{B}_{t}=-\frac{4 \pi}{c} \sigma_{H}^{t} \mathbf{E}=-(2 n+1) \frac{e^{2}}{\hbar c} \mathbf{E}
$$

in which CGS units are reintroduced. From this formula we can see that the magnetic field induced by an electric field is proportional to the electric field, where the response coefficient is quantized in odd multiples of the fine structure constant. When the magnetization of the side surface is reversed, the magnetic field induced is also reversed, as is expected from time-reversal symmetry. Combined with the conventional, non-topological response, we obtain $\mathbf{B}=\mathbf{H}+4 \pi \mathbf{M}-(2 n+1) \frac{e^{2}}{\hbar c} \mathbf{E}$, or

$$
\mathbf{H}=\mathbf{B}-4 \pi \mathbf{M}+(2 n+1) \frac{e^{2}}{\hbar c} \mathbf{E}
$$

Similar induction between electric and magnetic fields also occurs when a magnetic field is applied. Consider the process of applying a magnetic field $B$ parallel to the cylinder as shown in Fig. 15 (b). When the magnetic field is turned on from zero, a circulating electric field parallel to the side surface is generated, which then induces a Hall current $j \propto d B / d t$ parallel or anti-parallel to the magnetic field. Consequently, a charge density proportional to $B$ is accumulated on the top and bottom surfaces, so that a magnetic field induces an electric field parallel to it. By solving Maxwell equations, such a topological contribution to the electric field is obtained (a)

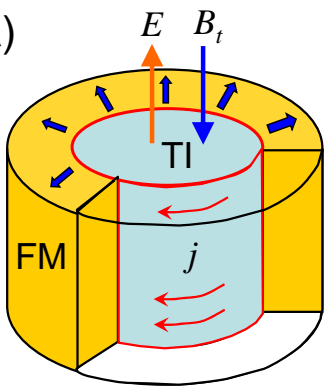

(c)

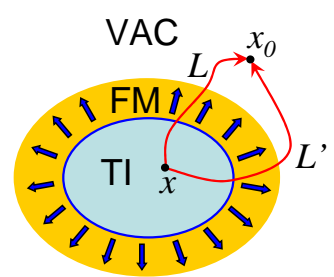

(b)

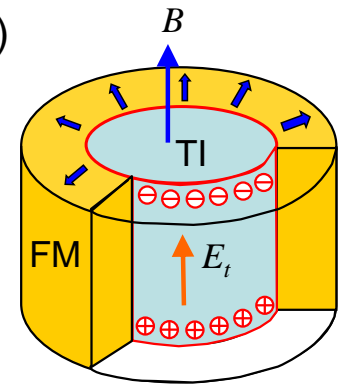

(d)

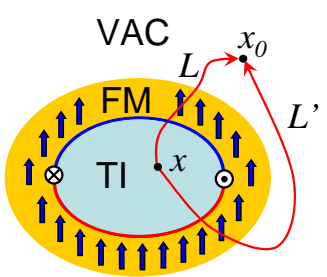

FIG. 15: (a) Illustration of the magnetic field induced by an electric field in a cylinder geometry. The magnetization of the FM layer points outward from the side surface of the TI, and a circulating current is induced by the electric field. (b) Illustration of the electric field induced by a perpendicular magnetic field in the same geometry. " $\oplus$ " and " $\ominus$ " indicate the positive and negative charge induced by the magnetic field on the top and bottom surface, respectively. (c) Illustration of a topological insulator with fully gapped surface states induced by a hedgehog magnetization configuration. $P_{3}$ of the topological insulator is well-defined since the integral along any two pathes $L$ and $L^{\prime}$ gives the same $\Delta P_{3}=P_{3}(\vec{x})-P_{3}\left(\vec{x}_{0}\right)$. (d) Illustration of a topological insulator with parallel magnetization on the surface. A one-d domain wall between two-d surface regions with different Hall conductance (as shown by red and blue) is induced, which carries chiral edge states as shown by $\otimes$ and $\odot$. In this case the $P_{3}$ of the topological insulator cannot be determined, since two different pathes $L$ and $L^{\prime}$ lead to different $\Delta P_{3}$.

as

$$
\mathbf{E}_{t}=(2 n+1) \frac{e^{2}}{\hbar c} \mathbf{B} .
$$

Combined with the conventional, non-topological response, we obtain:

$$
\mathbf{D}=\mathbf{E}+4 \pi \mathbf{P}-(2 n+1) \frac{e^{2}}{\hbar c} \mathbf{B}
$$

The conventional Maxwell's equations, supplemented by the constituent relations (101) and (99) give the complete description of the electrodynamics of the $3 \mathrm{D}$ topological insulators.

An alternative description is to use the conventional constituent relations $\mathbf{D}=\mathbf{E}+4 \pi \mathbf{P}$ and $\mathbf{H}=\mathbf{B}-4 \pi \mathbf{M}$, and work with a set of Maxwell's equations modified by the topological term. The total action of the electromagnetic field including the topological term is given by (80):

$$
S_{\text {tot }}=S_{\text {Maxwell }}+S_{\text {topo }}
$$




$$
\begin{aligned}
= & \int d^{3} x d t\left[\frac{1}{16 \pi} F_{\mu \nu} F^{\mu \nu}+\frac{1}{2} F_{\mu \nu} \mathcal{P}^{\mu \nu}-\frac{1}{c} j^{\mu} A_{\mu}\right] \\
& +\frac{\alpha}{16 \pi} \int d^{3} x d t P_{3} \epsilon^{\mu \nu \sigma \tau} F_{\mu \nu} F_{\sigma \tau}
\end{aligned}
$$

in which $\alpha \equiv e^{2} / \hbar c$ is the fine structure constant, and $\mathcal{P}^{0 i}=P^{i}$ and $\mathcal{P}^{i j}=\epsilon^{i j k} M_{k}$ are the electric and magnetic polarization vectors, respectively. The equations of motion are obtained by variation of the action over $A^{\mu}$ as

$$
\frac{1}{4 \pi} \partial_{\nu} F^{\mu \nu}+\partial_{\nu} \mathcal{P}^{\mu \nu}+\frac{\alpha}{4 \pi} \epsilon^{\mu \nu \sigma \tau} \partial_{\nu}\left(P_{3} F_{\sigma \tau}\right)=\frac{1}{c} j^{\mu} .
$$

These equations can also be written in the more familiar component form as

$$
\begin{aligned}
& \nabla \cdot \mathbf{D}=4 \pi \rho+2 \alpha\left(\nabla P_{3} \cdot \mathbf{B}\right) \\
& \nabla \times \mathbf{H}-\frac{1}{c} \frac{\partial \mathbf{D}}{\partial t}=\frac{4 \pi}{c} \mathbf{j}-2 \alpha\left(\left(\nabla P_{3} \times \mathbf{E}\right)+\frac{1}{c}\left(\partial_{t} P_{3}\right) \mathbf{B}\right) \\
& \nabla \times \mathbf{E}+\frac{1}{c} \frac{\partial \mathbf{B}}{\partial t}=0 \\
& \nabla \cdot \mathbf{B}=0
\end{aligned}
$$

where $\mathbf{D}=\mathbf{E}+4 \pi \mathbf{P}$ and $\mathbf{H}=\mathbf{B}-4 \pi \mathbf{M}$ include only the non-topological contributions. These are the equations of motion of axion electrodynamics $50,51,52$. By shifting the topological terms to the left-hand side, and redefining $\mathbf{D}$ and $\mathbf{H}$ according to (101) and (99), and taking $P_{3}=n+\frac{1}{2}$, we recover the conventional Maxwell's equations, but with modified constituent relations, thus demonstrating the equivalence to the formulation given above. The quantization of the TME effect in odd units of the fine structure constant is a deeply profound quantization phenomenon in condensed matter physics. The flux quantization inside a superconductor determines the fundamental constant $h c / e$, while the quantization of the Hall resistance determines the fundamental constant $h / e^{2}$. To date, there has been no other known quantization phenomenon in units of the dimensionless fine structure constant $\alpha=e^{2} / \hbar c$.

It should be emphasized that the value of $P_{3}$ in the topological insulator can only be determined when a magnetization is applied to open a gap on the surface. As shown in Fig. 15 (c), by defining a path $L$ from a reference point $\vec{x}_{0}$ deep in the vacuum, $P_{3}$ can be determined by $P_{3}(\vec{x})=\int_{\vec{x}_{0} L}^{\vec{x}} \overrightarrow{d l} \cdot \nabla P_{3}$. However, this definition only applies when the result does not depend on the choice of path. If a magnetic "shell" covered the surface of the topological insulator, with the magnetization outgoing everywhere on the interface, then the change of $P_{3}$ across the interface is the same for different points on the surface, such that the bulk $P_{3}$ is well-defined without dependence on the choice of path. In this case, Eqs. (99) and (101) are well-defined, and the integer part of $P_{3}$ can change if the magnetization direction is reversed. On the other hand, when there are domain walls on the surface, the integer part of $P_{3}$ is not well-defined in the bulk of the topological insulator, and Eqs. (99) and (101) do not apply, as shown in Fig. 15 (d). Physically, the failure of Eqs. (99) and (101) is simply due to the existence of a $\mathrm{QH}$ edge current on the domain wall, which requires the more general Maxwell equations Eqs. (104) including the contribution of the current. This analysis also provides a new picture of the surface $\mathrm{QH}$ effect, that is, the $\mathrm{QH}$ effect on the surface is carried by the chiral edge states living on vortex rings of the $P_{3}$ field. It is only when there are no vortex rings of $P_{3}$ on the surface, that the surface is fully gapped and the electro-magnetic response is simply given by Eqs. (99) and (101).

\section{(3) Low-frequency Faraday rotation.}

The TME effect can be experimentally observed in the settings discussed above, by applying an electric field through a capacitor, and measuring the magnetic field by a SQUID device. Alternatively, we consider the experiment of Faraday or Kerr rotation. The modified Maxwell equations (104) can be applied to another phenomenonphoton propagation in the system ${ }^{50}$. It should be noted that the effective theory (102) only applies in the lowenergy limit $E \ll E_{g}$, where $E_{g}$ is the gap of the surface state. Thus, to detect the topological phenomena we should study the low frequency photons with $\omega \ll E_{g} / \hbar$. Consider a FM-TI interface at $z=0$, as shown in Fig. 16 . Normally incident, linearly-polarized light can be written as:

$$
\mathbf{A}(z, t)=\left\{\begin{array}{cc}
\mathbf{a} e^{i(-k z-\omega t)}+\mathbf{b} e^{i(k z-\omega t)}, & z>0 \\
\mathbf{c} e^{i\left(-k^{\prime} z-\omega t\right)}, & z<0
\end{array}\right.
$$

in which $k=\omega / v$ and $k^{\prime}=\omega / v^{\prime}$ are the wavevectors of the photon in the $z>0$ and $z<0$ region, respectively. The $\nabla P_{3}$ terms in Eq. (104) contribute a non-conventional boundary condition at $z=0$. Define $\nabla P_{3}=\Delta \hat{\mathbf{z}} \delta(z)($ with $\Delta-1 / 2 \in \mathbb{Z})$, the boundary conditions are given by

$$
\begin{aligned}
\mathbf{a}+\mathbf{b} & =\mathbf{c} \\
\hat{\mathbf{z}} \times\left[k(-\mathbf{a}+\mathbf{b}) / \mu+k^{\prime} \mathbf{c} / \mu^{\prime}\right] & =-\frac{2 \alpha \Delta \omega}{c} \mathbf{c}
\end{aligned}
$$

in which the dimensionless constants $\epsilon, \epsilon^{\prime}$ and $\mu, \mu^{\prime}$ are the permittivity and permeability of the $z>0$ and $z<0$ materials, respectively. Denote $a_{ \pm}=a_{x} \pm i a_{y}$ and the same for $b_{ \pm}, c_{ \pm}$, the equations above lead to

$$
a_{+}=\frac{1}{2}\left[1+\frac{k^{\prime} / \mu^{\prime}-2 i \alpha \Delta \omega / c}{k / \mu}\right] c_{+} .
$$

Consequently, when the incident wave $\mathbf{a}$ is linearly polarized, the transmission wave $\mathbf{c}$ is also linearly polarized, with the polarization plane rotated by an angle

$$
\theta_{\text {topo }}=\arctan \frac{2 \alpha \Delta}{\sqrt{\epsilon / \mu}+\sqrt{\epsilon^{\prime} / \mu^{\prime}}} .
$$

Here we always assume that the magnetization of the FM material is perpendicular to the $x y$ plane, so that $\mathbf{H}=$ $\mu \mathbf{B}$ holds for in-plane magnetic fields. In the simplest 
(a)

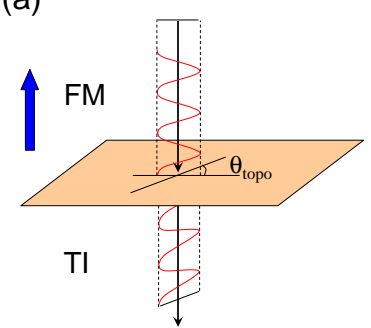

(b)

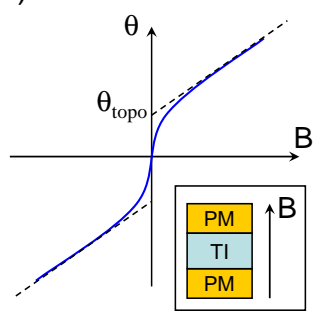

Experimentally, the main difficulty of measuring such an effect comes from the low-frequency constraint $\omega \ll$ $E_{g} / \hbar$. For a typical value $E_{g}=10 \mathrm{meV}$ we get $f \equiv$ $\omega / 2 \pi \ll 2.4 \mathrm{THz}$, which is in the far infrared or microwave region. In principle, it is possible to find a topological insulator with a larger gap which can support an accurate measurement of Faraday rotation. Similar proposals as above can also be worked out for the rotation of reflected wave (Kerr effect).

FIG. 16: (a) Illustration of the Faraday rotation $\theta_{\text {topo }}$ on the interface between a ferromagnet (or equivalently, a paramagnet in magnetic field) and a topological insulator. (b) Illustration of the total Faraday rotation angle $\theta$ as a function of magnetic field $B$ in the sandwich structure as shown in the inset. The zero-field extrapolation of $\theta(B)$ determines the topological term $\theta_{\text {topo }}$.

case $\mu, \mu^{\prime} \simeq 1, \epsilon, \epsilon^{\prime} \simeq 1$ and $\Delta=1 / 2$, we get $\theta \simeq \alpha \simeq$ $7.3 \times 10^{-3} \mathrm{rad}$.

Since the ferromagnetic material itself also induces a Faraday rotation, its necessary to distinguish these two contributions in order to measure the topological contribution (106). Replace the FM layers by paramagnetic materials with large susceptibilities, and apply an external magnetic field to polarize them. In this case the magnetization is proportional to magnetic field, such that the Faraday rotation contributed by the bulk is also proportional to magnetic field. The net Faraday rotation is given by $\theta=\theta_{\text {topo }}^{(t)}+\theta_{\text {topo }}^{(b)}+\theta_{\text {bulk }}$, which has the following dependence on the magnetic field:

$$
\theta(B)=u B+2 \operatorname{sgn}(B) \arctan \frac{\alpha}{\sqrt{\epsilon / \mu}+\sqrt{\epsilon^{\prime} / \mu^{\prime}}}(107)
$$

Consequently, the topological contribution can be obtained by measuring $\theta(B)$ at different applied magnetic fields and extracting the linear extrapolation of $\theta(B)$ as $B \rightarrow 0^{+}$.

\section{DIMENSIONAL REDUCTION TO $(2+1)$-D}

By carrying out the same dimensional reduction procedure once more, we can obtain the topological effective theory for TRI $(2+1)$-d insulators. Additionally a $Z_{2}$ classification can be defined for $(2+1)$-d TRI insulators, which is in exact analogy to the $Z_{2}$ classification of $(0+1)$-d particle-hole symmetric insulators. We will show that the $(2+1)-d Z_{2}$ nontrivial phase corresponds to the QSH insulator proposed recently $16,17,20,21$, and study the physical consequences of the effective theory.

\section{A. Effective action of $(2+1)$-d insulators}

In Sec. IVA we have seen how a $(3+1)$-d insulator with a parameter field $\theta(\vec{x}, t)$ is related to a $(4+1)$-d insulator through dimensional reduction. In the same way, two parameter fields can be defined to obtain the dimensional reduction from $(4+1)$-d to $(2+1)$-d. In the following we will still take the lattice Dirac model as a canonical example to show the dimensional reduction procedure and derive the effective theory.

Starting from the lattice Dirac model (72) and choosing a special gauge vector configuration satisfying $A_{n, n+\hat{i}}=A_{n+\hat{w}, n+\hat{w}+\hat{i}}=A_{n+\hat{z}, n+\hat{z}+\hat{i}}$, (so that the gauge vector is homogeneous along $z$ and $w$ ) we obtain the Hamiltonian

$$
\begin{aligned}
H[A]= & \sum_{k_{z}, k_{w}, \mathbf{x}} \sum_{s=1,2}\left[\psi_{\mathbf{x} ; k_{z}, k_{w}}^{\dagger}\left(\frac{c \Gamma^{0}-i \Gamma^{s}}{2}\right) e^{i A_{\mathbf{x}, \mathbf{x}+\hat{s}}} \psi_{\mathbf{x}+\hat{s} ; k_{z}, k_{w}}+h . c .\right]+\sum_{k_{z}, k_{w}, \mathbf{x}} \sum_{s=1,2} \psi_{\mathbf{x} ; k_{z}, k_{w}}^{\dagger} \\
& \cdot\left[\sin \left(k_{z}+A_{\mathbf{x} 3}\right) \Gamma^{3}+\sin \left(k_{w}+A_{\mathbf{x} 4}\right) \Gamma^{4}+\left(m+c \cos \left(k_{z}+A_{\mathbf{x} 3}\right)+c \cos \left(k_{w}+A_{\mathbf{x} 4}\right)\right) \Gamma^{0}\right] \psi_{\mathbf{x} ; k_{z}, k_{w}}
\end{aligned}
$$

in which $\mathbf{x}=(x, y)$ is the two-dimensional coordinate. As in the $(3+1)$-d case, the gauge fields in the $z$ and $w$ directions can be replaced by parameter fields $\left(k_{z}+A_{\mathbf{x} 3}\right) \rightarrow \theta_{\mathbf{x}}$ and $\left(k_{w}+A_{\mathbf{x} 4}\right) \rightarrow \varphi_{\mathbf{x}}$, resulting in the parameterized family of $(2+1)$-d Hamiltonians:

$$
\begin{aligned}
H_{2 \mathrm{D}}[A, \theta, \varphi]= & \sum_{\mathbf{x}, s}\left[\psi_{\mathbf{x}}^{\dagger}\left(\frac{c \Gamma^{0}-i \Gamma^{s}}{2}\right) e^{i A_{\vec{x}, \vec{x}+\hat{s}}} \psi_{\mathbf{x}+\hat{s}}+\text { h.c. }\right] \\
& +\sum_{\mathbf{x}, s} \psi_{\mathbf{x}}^{\dagger}\left[\sin \theta_{\mathbf{x}} \Gamma^{3}+\sin \varphi_{\mathbf{x}} \Gamma^{4}\right.
\end{aligned}
$$




$$
\left.+\left(m+c \cos \theta_{\mathbf{x}}+c \cos \varphi_{\mathbf{x}}\right) \Gamma^{0}\right] \psi_{\mathbf{x}} .
$$

By integrating out the fermion fields and expanding the resulting effective action around $A_{s}=0, \theta=\theta_{0}, \varphi=$ $\varphi_{0}$, the same nonlinear term shown in the Feynman diagram in Fig. 7 leads to the topological term

$$
S_{2 \mathrm{D}}=\frac{G_{2}\left(\theta_{0}, \varphi_{0}\right)}{2 \pi} \int d^{2} x d t \epsilon^{\mu \nu \rho} A_{\mu} \partial_{\nu} \delta \theta \partial_{\rho} \delta \varphi
$$

in which the coefficient $G_{2}\left(\theta_{0}, \varphi_{0}\right)$ is determined by the same correlation function as Eq. (53), but without the integrations over $k_{z}, k_{w}$ :

$$
\begin{aligned}
G_{2}\left(\theta_{0}, \varphi_{0}\right) & =\frac{2 \pi}{3} \int \frac{d^{2} k d \omega}{(2 \pi)^{3}} \operatorname{Tr} \epsilon^{\mu \nu \rho}\left[\left(G \frac{\partial G^{-1}}{\partial q^{\mu}}\right)\left(G \frac{\partial G^{-1}}{\partial q^{\nu}}\right)\left(G \frac{\partial G^{-1}}{\partial q^{\rho}}\right)\left(G \frac{\partial G^{-1}}{\partial \theta_{0}}\right)\left(G \frac{\partial G^{-1}}{\partial \varphi_{0}}\right)\right] \\
& =\frac{1}{4 \pi} \int d^{2} k \epsilon^{i j} \operatorname{Tr}\left[2 f_{i \theta} f_{j \varphi}-f_{i j} f_{\theta \varphi}\right]
\end{aligned}
$$

in which $\mu, \nu, \rho=0,1,2, i, j=1,2, q^{\mu}=\left(\omega, k_{x}, k_{y}\right)$, and the Berry curvature is defined in the four-dimensional parameter space $\left(k_{x}, k_{y}, \theta, \varphi\right)$. The coefficient $G_{2}\left(\theta_{0}, \varphi_{0}\right)$ satisfies the sum rule

$$
\int G_{2}\left(\theta_{0}, \varphi_{0}\right) d \theta_{0} d \varphi_{0}=2 \pi C_{2} .
$$

To simplify the expression further, the Chern-Simons form $\mathcal{K}^{A}$ in Eq. (78) can be introduced again. Here $A$ runs over $k_{x}, k_{y}, \theta, \varphi$, and $G_{2}\left(\theta_{0}, \varphi_{0}\right)$ can be written in terms of $\mathcal{K}^{A}$ as

$G_{2}\left(\theta_{0}, \varphi_{0}\right)=-2 \pi \int d^{2} k\left(\partial_{x} \mathcal{K}^{x}+\partial_{y} \mathcal{K}^{y}+\partial_{\theta} \mathcal{K}^{\theta}+\partial_{\varphi} \mathcal{K}^{\varphi}\right)$.

Similar to the $(3+1)$-d case, the momentum derivative terms $\partial_{(x, y)} \mathcal{K}^{(x, y)}$ lead to vanishing contributions if $\mathcal{K}^{(x, y)}$ is single-valued, in which case $G_{2}$ can be expressed as

$$
G_{2}\left(\theta_{0}, \varphi_{0}\right)=\partial_{\theta} \Omega_{\varphi}-\partial_{\varphi} \Omega_{\theta},
$$

with

$$
\Omega_{\varphi}=-2 \pi \int d^{2} k \mathcal{K}^{\theta}, \Omega_{\theta}=2 \pi \int d^{2} k \mathcal{K}^{\varphi} .
$$

Notice that

$$
\begin{aligned}
-\mathcal{K}^{\theta}= & -\frac{1}{16 \pi^{2}} \epsilon^{i j} \operatorname{Tr}\left[\left(f_{i j}-\frac{1}{3}\left[a_{i}, a_{j}\right]\right) \cdot a_{\varphi}\right. \\
& \left.-2\left(f_{i \varphi}-\frac{1}{3}\left[a_{i}, a_{\varphi}\right]\right) \cdot a_{j}\right]
\end{aligned}
$$

and similarly for $\mathcal{K}^{\varphi}$. We know that the vector $\Omega=$ $\left(\Omega_{\theta}, \Omega_{\varphi}\right)$ has the correct transformation properties of a gauge vector potential under the coordinate transformations of the parameter space $(\theta, \varphi)$, and also under gauge transformations of the wave functions. Consequently, when the parameters $\theta=\theta\left(x^{\mu}\right)$ and $\varphi=\varphi\left(x^{\mu}\right)$ have smooth dependence on space-time coordinates, an effective gauge vector potential $\Omega_{\mu}$ can be defined in $(2+1)$-d space-time as

$$
\Omega_{\mu} \equiv \Omega_{\theta} \partial_{\mu} \delta \theta+\Omega_{\varphi} \partial_{\mu} \delta \varphi
$$

the gauge curvature of which is related to $G_{2}$ as

$$
\begin{aligned}
\partial_{\mu} \Omega_{\nu}-\partial_{\nu} \Omega_{\mu} & =\left(\partial_{\theta} \Omega_{\varphi}-\partial_{\varphi} \Omega_{\theta}\right)\left(\partial_{\mu} \delta \theta \partial_{\nu} \delta \varphi-\partial_{\nu} \delta \theta \partial_{\mu} \delta \varphi\right) \\
& =G_{2}\left(\partial_{\mu} \delta \theta \partial_{\nu} \delta \varphi-\partial_{\nu} \delta \theta \partial_{\mu} \delta \varphi\right) .
\end{aligned}
$$

Mathematically, $G_{2}$ is a density of second Chern form in the 2D parameter space $(\theta, \phi)$, and $(\theta, \varphi)=$ $\left(\theta\left(x^{\mu}\right), \varphi\left(x^{\mu}\right)\right)$ defines a smooth map from the $(2+1)$ $\mathrm{d}$ space-time manifold to the $2 \mathrm{D}$ parameter space. The curvature of the gauge potential $\Omega_{\mu}$ is the pullback of $G_{2}$ to $(2+1)$-d space-time. By making use of Eq. (113) the effective action (109) can be rewritten in a parameterindependent form:

$$
S_{2 \mathrm{D}}=\frac{1}{2 \pi} \int d^{2} x d t \epsilon^{\mu \nu \tau} A_{\mu} \partial_{\nu} \Omega_{\tau}
$$

The physical consequences of the effective theory (115) can be understood by studying the response equation:

$$
j^{\mu}=\frac{1}{2 \pi} \epsilon^{\mu \nu \rho} \partial_{\nu} \Omega_{\rho} .
$$

As will be shown in the next subsection, Eq. (116) is the fundamental response equation for the QSH effect, which takes the form similar to the fundamental response equation for $\mathrm{QH}$ effect (8), with the replacement of the external gauge field by a effective Berry's phase gauge field. In this sense, our formalism provides a unifying theory for both effects. This type of relationship between different types of topological insulators will be discussed in more detail in section VI.

It is worth to noting that the response equation (116) can be expressed in an explicit form for the Dirac model 
(108). According to Eq. (64), the momentum-space second Chern number of the $(4+1)$-d Dirac model $h(\mathbf{k})=\sum_{a} d_{a}(\mathbf{k}) \Gamma^{a}$ is equal to the winding number of $\hat{\mathbf{d}}(\mathbf{k})$ on the unit sphere $S^{4}$. Correspondingly, the Hamiltonian of the $(2+1)$-d Dirac model (108) with constant $\theta$ and $\varphi$ has the form $h(\mathbf{k}, \theta, \varphi)=\sum_{a} d_{a}(\mathbf{k}, \theta, \varphi) \Gamma^{a}$, so the correlation function $G_{2}$ defined in Eq. (110) can be obtained as

$$
G_{2}(\theta, \varphi)=\frac{3}{4 \pi} \int d^{2} k \epsilon^{a b c d e} \frac{d_{a} \partial_{k_{x}} d_{b} \partial_{k_{y}} d_{c} \partial_{\theta} d_{d} \partial_{\varphi} d_{e}}{|\mathbf{d}(\mathbf{k}, \theta, \varphi)|^{5}} .
$$

Thus the curvature of effective gauge vector potential $\Omega_{\mu}$ is expressed as

$\partial_{\mu} \Omega_{\nu}-\partial_{\nu} \Omega_{\mu}=3 \epsilon^{a b c d e} \int \frac{d^{2} k}{4 \pi} \frac{d_{a} \partial_{k_{x}} d_{b} \partial_{k_{y}} d_{c} \partial_{\mu} d_{d} \partial_{\nu} d_{e}}{|\mathbf{d}(\mathbf{k}, \theta, \varphi)|^{5}}$

Now consider a slightly different version of lattice Dirac model given by

$$
\begin{aligned}
h(\mathbf{k}, \mathbf{n})= & \sin k_{x} \Gamma^{1}+\sin k_{y} \Gamma^{2}+\left(\cos k_{x}+\cos k_{y}-2\right) \Gamma^{0} \\
& +m \sum_{a=0,3,4} \hat{n}_{a} \Gamma^{a},
\end{aligned}
$$

in which $m>0$ and $\hat{\mathbf{n}}=\left(\hat{n}_{0}, \hat{n}_{3}, \hat{n}_{4}\right)$ is a $3 \mathrm{D}$ unit vector. For such a model the $\mathbf{d}$ vector can be decomposed as

$$
\mathbf{d}(\mathbf{k}, \theta, \varphi)=\mathbf{d}_{0}(\mathbf{k})+\left(\begin{array}{c}
0 \\
0 \\
m \hat{\mathbf{n}}
\end{array}\right)
$$

with $\mathbf{d}_{0}(\mathbf{k})=\left(\sin k_{x}, \sin k_{y}, 0,0, \cos k_{x}+\cos k_{y}-2\right)$. In the limit $m \ll 2$, the Hamiltonian has the continuum limit $h(\mathbf{k}, \hat{\mathbf{n}}) \simeq \sum_{a=1,2} k_{a} \Gamma^{a}+\sum_{b=0,3,4} m \hat{n}_{b} \Gamma^{b}$, which is the continuum $4 \times 4$ Dirac model with three possible mass terms. In this limit the integral over $\mathbf{k}$ in Eq. (117) can be explicitly carried out, leading to the following expression:

$$
\partial_{\mu} \Omega_{\nu}-\partial_{\nu} \Omega_{\mu}=\frac{1}{2} \hat{\mathbf{n}} \cdot \partial_{\mu} \hat{\mathbf{n}} \times \partial_{\nu} \hat{\mathbf{n}}
$$

which is the skyrmion density of the unit vector $\hat{\mathbf{n}}$. Combined with Eq. (116) we obtain the response equation for the Dirac model in the continuum limit:

$$
j^{\mu}=\frac{1}{8 \pi} \epsilon^{\mu \nu \tau} \hat{\mathbf{n}} \cdot \partial_{\mu} \hat{\mathbf{n}} \times \partial_{\nu} \hat{\mathbf{n}} .
$$

Eq. (118) describes a topological response where the charge density and current are equal to the skyrmion density and current, respectively. Such an equation can be considered as a $(2+1)$-d version of GoldstoneWilczek formula (31), which has been studied extensively in the literature $13,65,66,67,68$. Thus, through the discussion above we have shown that the topological response formula(118) of $(2+1)$-d Dirac fermions is a special example of the generic response equation (116).

To understand the physics described by Eq. (116), consider the lattice Dirac model in Eq. (108) with an (a)

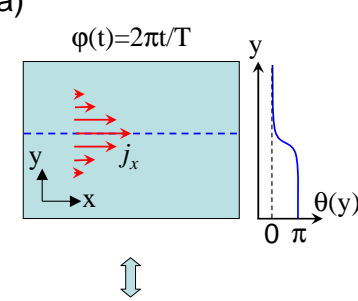

(b)

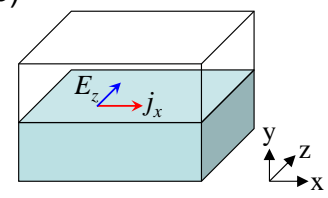

(c)

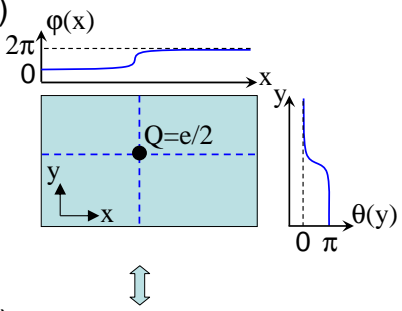

(d)

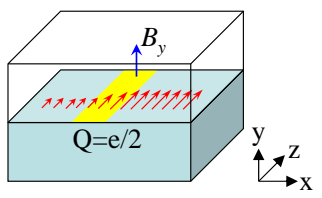

FIG. 17: (a) Illustration of the charge pumping effect in the $(2+1)$-d lattice Dirac model with spatial dependent $\theta=\theta(y)$ and time-dependent $\varphi=\varphi(t)$. This effect is the dimensional reduction of the domain-wall QH effect in the $(3+1)$-d system as shown in Fig. (b), in which $\partial_{t} \varphi$ plays the role of electric field $E_{z}$. (c) Illustration of the half charge trapped at the crossing point of the $\theta$ and $\varphi$ domain walls. This effect is the dimensional reduction of the charge trapped on the domain wall by a magnetic field, as shown in Fig. (d). $\varphi(x)$ corresponds to the gauge vector potential $A_{z}$ as shown by red arrows, the curvature of which leads to a magnetic field $B_{y}$ in the yellow region with net flux $2 \pi$. Due to the half quantized Hall conductance of the $\theta$ domain wall, such a magnetic flux induces a half charge.

adiabatic time-evolution of $\varphi(t)=2 \pi t / T$, and a spatial domain wall configuration of $\theta(\vec{x})$ :

$$
\theta(y)=\frac{\pi}{2}\left[1+\tanh \left(\frac{y}{\xi}\right)\right]
$$

as shown in Fig. 17 (a). According to Eq. (116), the charge current along the domain wall is given by

$$
j_{x}=\frac{1}{2 \pi}\left(\partial_{y} \Omega_{t}-\partial_{t} \Omega_{y}\right)
$$

When the parameter $\varphi$ evolves adiabatically from 0 to $2 \pi$, the net charge flowing across the line $x=0$ is given by $\int d t I_{x}=\int d t d y\left(\partial_{y} \Omega_{t}-\partial_{t} \Omega_{y}\right) / 2 \pi=\int_{0}^{\pi} d \theta \int_{0}^{2 \pi} d \varphi\left(\partial_{\theta} \Omega_{\varphi}-\right.$ $\left.\partial_{\varphi} \Omega_{\theta}\right) / 2 \pi$, which is the integration of the second Chern form $\operatorname{Tr}\left[\epsilon^{A B C D} f_{A B} f_{C D}\right] / 32 \pi^{2}$ over the parameter range $\theta \in[0, \pi], \varphi \in[0,2 \pi]$, where $A, B, C, D=k_{x}, k_{y}, \theta, \varphi$. According to the discussion in the $(3+1)$-d case, we know that a magneto-electric polarization $P_{3}$ can be defined as

$$
P_{3}(\theta)=\int d^{2} k d \varphi \mathcal{K}^{\theta}=-\int d \varphi \Omega_{\varphi} / 2 \pi
$$

which implies $\int d t I_{x}=-\int_{0}^{\pi} d \theta \partial_{\theta} P_{3}(\theta)$. For $-4 c<m<$ $-2 c$, we have $\int_{0}^{\pi} d P_{3}(\theta)=1 / 2$, corresponding to the pumped charge $\Delta Q=\int d t I_{x}=1 / 2$. In summary, the physical consequence of the topological response equation (116) is to induce a topological charge pumping effect 
during the adiabatic evolution of $\varphi$, in which the charge pumped in one period is proportional to the spatial gradient of the magneto-electric polarization $P_{3}$ defined in $\left(k_{x}, k_{y}, \varphi\right)$ space. Specifically, a charge $e / 2$ is pumped along each $\Delta \theta=\pi$ domain wall of $\theta$ when $\varphi$ evolves from 0 to $2 \pi$.

Such a charge pumping effect can also be viewed as the dimensional reduction of the half $\mathrm{QH}$ effect on the $\theta$ domain wall of $(3+1)$-d lattice Dirac model, which has been studied in Sec. IVB, This dimensional reduction procedure is in exact analogy with the usual reduction from the $(2+1)$-d $\mathrm{QH}$ effect to $(1+1)$-d quantized pumping effect studied in Sec. IIC. Similar to the latter case, a fractional charge effect can also be proposed in $(2+1)$-d according to Eq. (116). To show this effect, one can consider the same $\theta$ domain wall as shown above, and a $2 \pi$ domain wall of $\varphi$ along the $y$-direction $\varphi(\mathbf{x})=\pi[1+\tanh (x / \xi)]$, as shown in Fig. 17 (b). The charge density is given by $\rho=\left(\partial_{x} \Omega_{y}-\partial_{y} \Omega_{x}\right) / 2 \pi$. By integrating over the $x$-direction we obtain $\int \Omega_{x} d x=$ $\int \Omega_{\varphi} d \varphi=-2 \pi P_{3}(\theta)$, such that $\rho_{1 \mathrm{D}}=\int d x \rho=\partial_{y} P_{3}(\theta)$ and $\int d y \rho_{1 \mathrm{D}}=1 / 2$. Thus, a half charge is localized at the crossing of $\theta$ and $\varphi$ domain walls 69 . Such a fractional charge effect can also be understood through the dimensional reduction from $(3+1)$-d. The spatial dependence of $\varphi(x)$ corresponds to the spatial dependence of $k_{z}-A_{z}(x)$, which describes a magnetic field perpendicular to the 2D domain wall in $(3+1)$-d system. When $\varphi(x)$ has a $2 \pi$ domain wall, the net flux of the corresponding magnetic field is $2 \pi$, which thus induces a half charge as shown in Fig. 17 (b).

In summary, we have studied the physical consequences of the topological effective action (115) in a spatially and/or temporally inhomogeneous insulator. In the rest of this section we will show how to define a $Z_{2}$ topological invariant in $(2+1)$-d TRI insulators and study the physical properties of the $Z_{2}$ nontrivial phase- $\mathrm{QSH}$ phase - by applying the effective theory (115).

\section{B. $Z_{2}$ topological classification of TRI insulators}

In Sec. IID and IIE we have shown how a $Z_{2}$ classification of particle-hole invariant insulators can be defined in both $(1+1)$-d and $(0+1)$-d through dimensional reduction from the $(2+1)$-d $\mathrm{QH}$ effect. The second-Chernclass analogy of the $(2+1)-\mathrm{d}$ QH effect is the $(4+1)$-d QH effect ${ }^{14}$ described by the Chern-Simons theory (52), which then leads to the $Z_{2}$ classification of TRI insulators in $(3+1)$-d, as shown in Sec. IVC Following this line of reasoning, it is straightforward to see that a $Z_{2}$ classification can be defined for $(2+1)$-d TRI insulators, as an analog of $(0+1)$-d particle-hole symmetric insulators. In this subsection we will sketch the demonstration of such a topological classification without going into detail since the derivation here is exactly parallel to that in Sec. IIE

First of all, for two TRI $(2+1)$-d insulators $h_{1}(\mathbf{k}), h_{2}(\mathbf{k})$ an adiabatic interpolation $h(\mathbf{k}, \theta)$ can be defined, satisfying

$$
\begin{aligned}
h(\mathbf{k}, 0) & =h_{1}, h(\mathbf{k}, \pi)=h_{2} \\
T^{\dagger} h(-\mathbf{k},-\theta) T & =h^{T}(\mathbf{k}, \theta),
\end{aligned}
$$

Since $h(\mathbf{k}, \theta)$ corresponds to the Hamiltonian of a $(3+1)$ d TRI insulator, a $Z_{2}$ topological quantity $N_{3}[h(\mathbf{k}, \theta)]=$ \pm 1 can be defined as shown in Sec. [VC] The key point to defining a $Z_{2}$ invariant for the $(2+1)$-d Hamiltonians $h_{1}, h_{2}$ is to demonstrate the independence of $N_{3}[h(\mathbf{k}, \theta)]$ on the choice of $h(\mathbf{k}, \theta)$. Consider two different parameterizations $h(\mathbf{k}, \theta)$ and $h^{\prime}(\mathbf{k}, \theta)$. An interpolation $g(\mathbf{k}, \theta, \varphi)$ can be defined between them which satisfies

$$
\begin{aligned}
g(\mathbf{k}, \theta, 0) & =h(\mathbf{k}, \theta), g(\mathbf{k}, \theta, \pi)=h^{\prime}(\mathbf{k}, \theta) \\
g(\mathbf{k}, 0, \varphi) & =h_{1}(\mathbf{k}), g(\mathbf{k}, \pi, \varphi)=h_{2}(\mathbf{k}) \\
g^{T}(\mathbf{k}, \theta, \varphi) & =T^{\dagger} g(-\mathbf{k},-\theta,-\varphi) T .
\end{aligned}
$$

$g(\mathbf{k}, \theta, \varphi)$ corresponds to a $(4+1)$-d insulator Hamiltonian, for which a second Chern number $C_{2}[g]$ is defined. By its definition, the "second Chern parity" $N_{3}$ of $h(\mathbf{k}, \theta)$ and $h^{\prime}(\mathbf{k}, \theta)$ satisfies $N_{3}[h] N_{3}\left[h^{\prime}\right]=(-1)^{C_{2}[g]}$. At the same time, $g(\mathbf{k}, \theta, \varphi)$ can also be considered as an interpolation between $\theta=0$ and $\theta=\pi$ systems, i.e., between $g(\mathbf{k}, 0, \varphi) \equiv h_{1}(\mathbf{k})$ and $g(\mathbf{k}, \pi, \varphi) \equiv$ $h_{2}(\mathbf{k})$. Since $h_{1,2}(\mathbf{k})$ are both independent of $\varphi$, the $\varphi$ component of the Berry's phase gauge field vanishes for $g(\mathbf{k}, 0, \varphi)$ and $g(\mathbf{k}, \pi, \varphi)$. Consequently, it can be shown that $(-1)^{C_{2}[g]}=N_{3}[g(\mathbf{k}, 0, \varphi)]=N_{3}[g(\mathbf{k}, \pi, \varphi)]=1$, so that $N_{3}[h] N_{3}\left[h^{\prime}\right]=1$ for any two interpolations $h$ and $h^{\prime}$. Thus, we have shown that the $Z_{2}$ quantity $N_{2}\left[h_{1}(\mathbf{k}), h_{2}(\mathbf{k})\right] \equiv N_{3}[h(\mathbf{k}, \theta)]$ only depends on the $(2+1)$-d Hamiltonians $h_{1}$ and $h_{2}$. By defining a constant Hamiltonian $h_{0}(\mathbf{k})=h_{0}$ as reference, all $(2+1)$-d TRI insulators are classified by the value of $N_{2}\left[h_{0}, h(\mathbf{k})\right]$. An insulator with $N_{2}\left[h_{0}, h\right]=-1$ cannot be adiabatically deformed to the trivial Hamiltonian $h_{0}$ without breaking time-reversal symmetry.

In the next subsection, the physical properties of the $Z_{2}$ non-trivial insulator defined here will be studied. We will see that the $Z_{2}$ non-trivial insulator defined here has non-trivial edge dynamics, and corresponds to the QSH insulator studied in the literature $\frac{16,17,20,21}{}$. Compared to the former definition of the $Z_{2}$ topological classification ${ }^{16,23,26}$, our definition has the advantage of providing a direct relationship between the topological quantum number and the physical response properties of the system.

\section{Physical properties of the $Z_{2}$ nontrivial insulators}

Similar to the $(3+1)$-d case, the topological properties of a $Z_{2}$ nontrivial insulator lead to non-trivial edge state dynamics described by the effective theory (115), or equivalently, the response equation (116). The edge of a 
$Z_{2}$ nontrivial insulator is equivalent to a domain wall between a nontrivial insulator and a trivial insulator (since the vacuum can be considered as a trivial insulator with a large gap). Thus, in the following we will focus on the domain wall between a nontrivial system with Hamiltonian $h_{1}(\mathbf{k})$ and a trivial system with Hamiltonian $h_{0}$.

As discussed in the last subsection, an interpolation $h(\mathbf{k}, \theta)$ can be defined between $h_{0}$ and $h_{1}$ satisfying $h(\mathbf{k}, 0)=h_{0}, \quad h(\mathbf{k}, \pi)=h_{1}(\mathbf{k})$ and $T^{\dagger} h(-\mathbf{k},-\theta) T=$ $h^{T}(\mathbf{k}, \theta)$. Since $h_{1}$ is nontrivial, $h(\mathbf{k}, \theta)$ has to break timereversal symmetry for general $\theta$ to adiabatically connect $h_{1}$ to $h_{0}$. Making use of $h(\mathbf{k}, \theta)$, two different interfaces between $h_{1}$ and $h_{0}$ can be defined. Consider a spatially dependent $\theta$ given by

$$
\theta(x, y)=\frac{\pi}{2}\left[1-\tanh \left(\frac{y}{\xi}\right)\right] .
$$

Then the spatially dependent Hamiltonian $h(\mathbf{k}, \theta(y))$ and $h(\mathbf{k},-\theta(y))$ both describe a spatial domain wall between $h_{1}$ (for $y \ll-\xi$ ) and $h_{0}$ (for $y \gg \xi$ ). The only difference between these two Hamiltonians are the timereversal symmetry breaking terms around the interface. Now consider a more complicated interface, with

$$
h(\mathbf{k}, \mathbf{x})=\left\{\begin{array}{cc}
h(\mathbf{k}, \theta(y)), & x<0 \\
h(\mathbf{k},-\theta(y)), & x>0
\end{array}\right.
$$

as shown in Fig. 18 (a). In such a system, the timereversal symmetry on the interface is broken in opposite ways for $x>0$ and $x<0$, in the sense that $h^{T}(\mathbf{k},(x, y))=T^{\dagger} h(-\mathbf{k},(-x, y)) T$. Now we study the charge localized around the point $x=0, y=0$. For a loop $C$ enclosing this point as shown in Fig. 18 (a), the charge in the region $A$ enclosed by $C$ is given by Eq. (116) as

$$
Q=\frac{1}{2 \pi} \int_{A} d^{2} x\left(\partial_{x} \Omega_{y}-\partial_{y} \Omega_{x}\right)=\frac{1}{2 \pi} \oint_{C} \boldsymbol{\Omega} \cdot d \mathbf{l} .
$$

When the size of the loop is large enough compared to the boundary width $\xi$, such a loop integration is equivalent to an integration over $\theta$ from 0 to $2 \pi$, which leads to $Q=$ $\oint \Omega_{\theta} d \theta / 2 \pi=P_{3}[h(\mathbf{k}, \theta)]$. According to the definition of a $Z_{2}$ non-trivial insulator in the last section, $P_{3}[h(\mathbf{k}, \theta)]=$ $1 / 2 \bmod 1$ for any interpolation $h(\mathbf{k}, \theta)$ between $h_{0}$ and $h_{1}$. Consequently, the charge confined on the domain wall is $Q=(n+1 / 2) e$ with $n$ an integer depending on the details of the interface ${ }^{69}$.

To summarize, a time-reversal symmetry breaking term can be applied at the interface of trivial and nontrivial insulators. For a given interface described by Hamiltonian $h(\mathbf{k}, y)$, its time-reversal partner, $h^{\prime}(\mathbf{k}, y)=$ $T h^{T}(-\mathbf{k}, y) T^{\dagger}$, describes a different connecting condition at the interface. If the 1D interface is described by $h$ in one region and by $h^{\prime}$ in another region, then the domain wall between these two regions will trap a half-charge as a consequence of the non-trivial topology. To understand such a domain wall better, we can consider the case with (a)

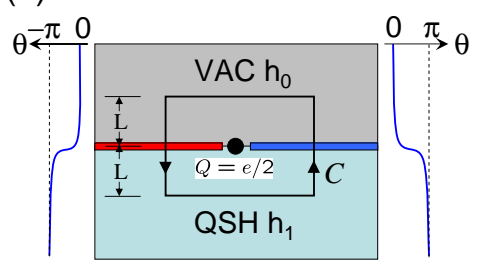

(b)

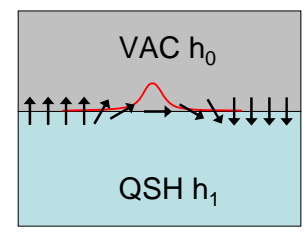

FIG. 18: (a) Illustration of an interface between the vacuum (VAC) with Hamiltonian $h_{0}(\mathbf{k})$ and a QSH insulator (QSH) with Hamiltonian $h_{1}(\mathbf{k})$. An interpolation $h(\mathbf{k}, \theta), \theta \in[0,2 \pi]$ can be defined between $h_{0}$ and $h_{1}$. On the left (right) half of the interface marked by red (blue), $\theta$ has a domain wall from 0 to $-\pi(\pi)$. A fractional charge $Q=e / 2$ is trapped on the domain wall between the red and blue interfaces, which can be calculated by an integration of $\Omega$ along the loop $L$ (see text). (b) Physical realization of the domain wall between two interfaces in (a) by an anti-phase domain wall of magnetic field or magnetization. The red curve shows schematically the charge density distribution.

a magnetic field as the time-reversal symmetry breaking term on the interface. When the magnetic field has an anti-phase domain wall as shown in Fig. 18 (b), a halfcharge must be trapped on the domain wall.

Moreover, one can also obtain the distribution of $1 \mathrm{D}$ charge density and current density on the interface by integrating the Eq. (116) only along the $y$-direction:

$$
\begin{aligned}
\rho_{1 \mathrm{~d}}(x) & =\frac{1}{2 \pi} \int_{-L}^{L} d y\left(\partial_{x} \Omega_{y}-\partial_{y} \Omega_{x}\right) \\
\mathrm{J} 1 \mathrm{~d}(x) & =\frac{1}{2 \pi} \int_{-L}^{L} d y\left(\partial_{y} \Omega_{t}-\partial_{t} \Omega_{y}\right)
\end{aligned}
$$

in which $L$ is a cut-off in the $y$-direction, satisfying $L \gg \xi$ so that the contribution to $\rho_{1 \mathrm{D}}$ and $j_{1 \mathrm{D}}$ from the region $|y|>L$ is negligible. According to the definitions (112) and (113) of the effective gauge vector potential $\Omega_{\mu}$, we know that $\Omega_{\mu}(x, y, t) \rightarrow 0$ for a point deep in the QSH or VAC region, i.e., when $|y| \rightarrow \infty$. Thus the expression of $1 \mathrm{D}$ density and current can be simplified to

$$
\rho_{1 \mathrm{D}}(x, t)=\partial_{x} P_{3}(x, t), j_{1 \mathrm{D}}(x, t)=-\partial_{t} P_{3}(x, t)
$$

with $P_{3}(x, t)=\int_{-L}^{L} d y \Omega_{y}(x, y, t) / 2 \pi$ the magneto-electric polarization defined in $\left(k_{x}, k_{y}, y\right)$ space. Eq. (120) is exactly the Goldstone-Wilczek formula 13 describing the charge fractionalization effect in the $(1+1)$-d Dirac model, and $2 \pi P_{3}(x, t)$ plays the role of the phase angle of the Dirac mass term. When the interfaces on the left and right sides of the domain wall are related by time-reversal symmetry, the change of $2 \pi P_{3}$ through the domain wall must be $(2 n+1) \pi$, which gives the half charge on the domain wall.

Such a relation between the interface and the $(1+1)-\mathrm{d}$ Dirac model can be understood more intuitively in terms 
(a)

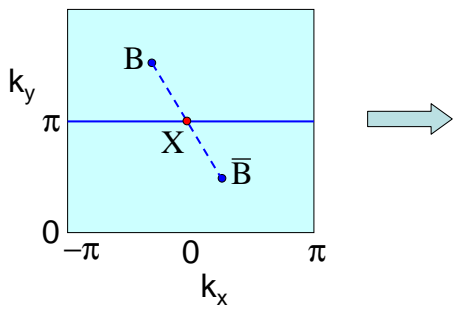

(b)

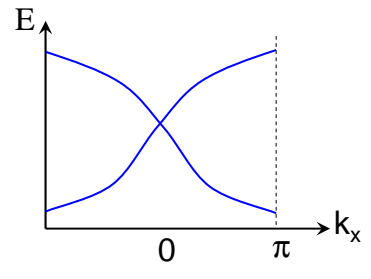

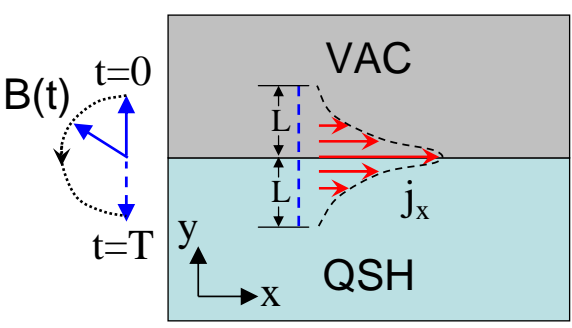

FIG. 19: Illustration of the dimensional reduction from the surface of a $(3+1)$-d topological insulator (a) to the edge of a $(2+1)$-d QSH insulator (b). The red and blue points in (a) are the positions of gapless $(2+1)$-d Dirac cones in the surface BZ. The blue line at $k_{y}=\pi$ defines the edge theory of a QSH insulator, as shown in Fig. (b).

of the edge effective theory $\underline{69}$. Similar to the relation between the edge theory of a $(4+1)$-d topological insulator and the reduced edge theory of a $(3+1)-d Z_{2}$ non-trivial insulator, we can obtain the edge theory of a $(2+1)$-d $Z_{2}$ nontrivial insulator from that of a $(3+1)$-d nontrivial insulator. The interpolation $h(\mathbf{k}, \theta)$ between $h_{1}$ and $h_{0}$ can be viewed as the Hamiltonian of a $(3+1)$-d TRI insulator, in which $\theta$ plays the role of $k_{z}$. Consider a specific point of the $(2+1)$-d boundary of the $(3+1)$-d system, say $y=0$. According to the discussion in Sec. IVC an odd number of $(2+1)$-d Dirac fermions are propagating on the boundary of the $(3+1)-d$ system. Due to time-reversal symmetry there must be an odd number of Dirac cones on the four time-reversal symmetric points, as shown in Fig. 12 (b). For a slice at $y=0$, the wavevector of the surface state is $\left(k_{x}, \theta\right)$. Consequently, when $\theta$ is considered to be a parameter, the surface energy spectrum $E\left(k_{x}, \theta\right)$ for a given $\theta$ describes the dispersion of $(1+1)$-d edge states of $(2+1)$-d insulators. Specifically, $\theta=0$ corresponds to the vacuum Hamiltonian $h_{0}$, which cannot support any non-trivial edge states. Thus, the Dirac cones at the time-reversal symmetric points can only appear at $\left(k_{x}, \theta\right)=(0, \pi)$ and $(\pi, \pi)$. To have the minimal odd number of Dirac cones in the $\left(k_{x}, \theta\right) \mathrm{BZ}$, there must be one gapless Dirac cone at $(0, \pi)$ or $(\pi, \pi)$, but not both, as shown in Fig. 19. In other words, the edge effective theory of a $(2+1)$-d nontrivial insulator is given by a gapless Dirac theory

$$
H=\int \frac{d k}{2 \pi} v \sum_{\sigma= \pm 1} \sigma k \psi_{k \sigma}^{\dagger} \psi_{k \sigma}
$$

where $\sigma= \pm 1$ means left and right movers, respectively. This edge theory agrees with the former descriptions of QSH edge states 18,19,26 and shows the equivalence of the $Z_{2}$ nontrivial insulator defined in this section and the QSH insulator.

Once the edge theory is obtained, it is easy to understand the charge fractionalization proposed above. Due to Kramers's degeneracy, any TRI perturbation cannot
FIG. 20: Illustration of quantized charge pumping on the boundary of a QSH insulator induced by a rotating magnetic field. During the time period $t \in[0, T]$ the magnetic field is rotated from $B$ to $-B$, and a half charge $Q=\int d t \int_{-L}^{L} d y j_{x}=$ $e / 2$ is pumped along $x$ direction. $L$ is taken as a cut-off with $j_{x}$ vanishingly small for $|y|>L$.

open a gap at the edge. Only when a magnetic field, or other time-reversal symmetry breaking term is applied, can a mass term $m_{x} \sigma_{x}+m_{y} \sigma_{y}$ be generated in the edge theory. Time-reversal symmetry also guarantees that the mass induced by opposite magnetic fields is exactly opposite. This implies that an anti-phase domain wall of the magnetic-field corresponds to a sign-change of the mass of the Dirac fermion. Thus, the edge state theory is described by the well-known Jackiw-Rebbi model ${ }^{39}$ or equivalently, the $\mathrm{Su}$-Schrieffer-Heeger mode ${ }^{\underline{9}}$. The study of the fractional charge in the edge theory approach and its experimental consequences have been presented in Ref. 69. Thus, we have seen that the effective theory we obtained from dimensional reduction agrees with the edge theory analysis, just like in the $(3+1)$-d case. The effective theory correctly describes the half-charge associated with a magnetic domain wall, which is a direct physical manifestation of the $Z_{2}$ quantum number.

A quantized charge pumping effect always accompanies a fractional charge effect and can be realized when a time-dependent T-breaking field is applied at the edge. If the system is described by a time-dependent Hamiltonian $h(\mathbf{k}, \mathbf{x}, t)$ which satisfies $h(\mathbf{k}, \mathbf{x}, t=0)=h(\mathbf{k}, \theta(y))$ and $h(\mathbf{k}, \mathbf{x}, t=T)=h(\mathbf{k},-\theta(y))$ with $\theta(y)$ the domain wall configuration discussed above, then the charge pumped through the interface during the time $t \in[0, T]$ is given by

$$
Q_{\text {pump }}=\int_{0}^{T} d t j_{1 \mathrm{D}}=-\left(P_{3}(T)-P_{3}(0)\right)=-\left(n+\frac{1}{2}\right) .
$$

In the example of an applied magnetic field, such a pumping process implies that a half charge is pumped when a magnetic field rotates adiabatically from $\mathbf{B}$ to $-\mathbf{B}$, as shown in Fig. 20. The experimental proposal of such a charge pumping effect is also discussed in Ref. 69 .

Besides providing a quantized response property of the QSH insulators, the fractional charge and charge pumping effects proposed here are a $(1+1)$-d version of electro-magnetic duality. In $(3+1)$-d, the electromagnetic duality gives rise to the Witten effect $\underline{61}$, where 
a magnetic monopole carries a charge $\Theta / 2 \pi$ and becomes a "dyon" when a topological $\Theta$-term is introduced in the Lagrangian 61.70 . Such an effect can occur in a $(3+1)$-d topological insulator where the charge of the dyon is $1 / 2$, as studied in Sec. IVB. In comparison, the magnetic domain wall on the boundary of $(2+1)$-d QSH insulator can be considered as a topological point defect of magnetic field in $(1+1)$-d, which also carries a half-charge. In this sense, if we consider the magnetic domain wall as a dynamical degree of freedom of the system, (e.g., when the magnetic domain wall is generated by a ferromagnetic stripe on top of the 2D QSH system) it can be considered as the $(1+1)$-d manifestation of dyons.

Interestingly, such an analogy can also be generalized to $(2+1)-d$, where the topological defect of magnetic field is a flux tube. Recently it has been shown that a $\pi$ flux tube threaded into a QSH insulator carries either charge $\pm e$, spin 0 or charge 0 , spin $1 / 2$, where the spin $0(1 / 2)$ is generically defined as a Kramers' singlet (doublet) under time-reversal symmetry 31,32 In other words, the $\pi$ flux tube becomes a dyon-like object and realizes spin-charge separation in $(2+1)$-d. Such a spin-charge separation phenomenon also provides an alternative definition of the $Z_{2}$ topological insulators in $(2+1)-\mathrm{d}^{31}$.

In summary, we have shown how a $Z_{2}$ classification of $(2+1)$-d TRI insulators is obtained, and how the physical properties of the $Z_{2}$ non-trivial insulator are described by the effective theory derived from dimensional reduction. Together with the discussion of $(3+1)$-d topological insulators in Sec. IV] we have seen that the nontrivial topology and its consequences in both $(3+1)$-d and $(2+1)$-d TRI systems have their origin in the nontrivial second Chern number in $(4+1)$-d. The dimensional reduction series $(4+1)$ - $d \rightarrow(3+1)$ - $d \rightarrow(2+1)$ - $d$ is in exact analogy of the lower dimensional one $(2+1)$ $\mathrm{d} \rightarrow(1+1)-\mathrm{d} \rightarrow(0+1)-\mathrm{d}$. In next section, we will develop the unified framework of dimensional reduction in generic dimensions, which contains the two series as simplest examples.

\section{UNIFIED THEORY OF TOPOLOGICAL INSULATORS}

\section{A. Phase space Chern-Simons theories}

Up to now, we have systematically studied several related topological phenomena, including the $(2+1)-\mathrm{d} \mathrm{QH}$ insulator with nontrivial first Chern number, the $(4+1)$-d topological insulator with nontrivial second Chern number, and their dimensional reductions. Comparing Sec. II with Secs. [II V] one can easily see the exact analogy between the two series of topological insulators: the $(2+1)-\mathrm{d}$ and $(4+1)-\mathrm{d}$ fundamental topological insulators are characterized by an integer - the first and second Chern number, respectively. Under a discrete symmetry (particle-hole symmetry for the $(2+1)$-d family and timereversal symmetry for the $(4+1)$-d family), a $Z_{2}$ topologi- cal classification can be defined for the lower dimensional descendent systems; the physical properties of which can be described by effective theories obtained from the dimensional reduction procedure. The main facts about these topological phenomena are summarized in Table —. In this section, we will show that the effective theories for all these systems share a universal form when written in phase space.

As a simple example, we first consider the effective theory (9) of the QH effect. Expanding the expression of the first Chern number explicitly, Eq. (9) can be expressed as

$$
S=\frac{1}{4 \pi} \int \frac{d^{2} k}{2 \pi} \epsilon^{i j} \operatorname{Tr}\left[\partial_{i} a_{j}\right] \int d t d^{2} x \epsilon^{\mu \nu \rho} A_{\mu} \partial_{\nu} A_{\rho}
$$

in which $i, j$ are indices 1,2 in momentum space and $\mu, \nu, \rho=0,1,2$ are space-time indices. Here and below, $A_{\mu}$ and $a_{i}$ stand for the external electromagnetic gauge field in real space and Berry's phase gauge field in momentum space, respectively. The trace is carried over all occupied energy levels. If we define the phase-space coordinate as $\mathbf{q}=\left(t, x, y, k_{x}, k_{y}\right)$ and the phase-space gauge potentials $\mathbf{A}=\left(A_{0}, A_{1}, A_{2}, 0,0\right), \mathbf{a}=\left(0,0,0, a_{1}, a_{2}\right)$, then the action above is equivalent to the following second Chern-Simons term:

$$
S_{2+1}=\frac{1}{8 \pi^{2}} \int d^{5} q \epsilon^{A B C D E} A_{A} \partial_{B} A_{C} \operatorname{Tr}\left[\partial_{D} a_{E}\right]
$$

where all capital roman indices e.g. $A, B, C \ldots$ run over the appropriate phase space coordinates. Since in this system $\mathbf{A}$ and $\mathbf{a}$ are always orthogonal to each other, such a reformulation seems trivial. However, it turns out to be helpful when considering the dimensional reduction procedure. As discussed earlier, dimensional reduction of the $(2+1)$-d system to $(1+1)$-d is defined by replacing, say, $k_{y}+A_{y}$ by a parameter $\theta(x, t)$, which in general is space-time dependent. Four changes are induced by this substitution:

1. $A_{y}$ is replaced by $\theta(x, t)-\theta_{0}$ with $\theta_{0}$ playing the role of $k_{y}$

2. $\partial / \partial k_{y}$ is replaced by $\partial / \partial \theta$

3. The Berry's phase gauge field $a_{k_{y}}^{\alpha \beta}=$ $-i\left\langle\alpha ; \mathbf{k}\left|\partial_{k_{y}}\right| \beta ; \mathbf{k}\right\rangle$ is replaced by $a_{\theta}^{\alpha \beta}=$ $-i\left\langle\alpha ; k_{x}, \theta\left|\partial_{\theta}\right| \beta ; k_{x}, \theta\right\rangle$

4. The integrations $\int d y$ and $\int d k_{y} / 2 \pi$ are removed from the effective action.

By making these substitutions, the effective action of the $(1+1)$-d system can be obtained. To help understand the general dimensional reduction procedure, we show the derivation of the $(1+1)$-d effective theory explicitly. For simplicity, one can start from Eq. (122). Note that the 


\begin{tabular}{|c|c|c|c|c|c|}
\hline & Dimension & $\begin{array}{c}\text { Topological quantum } \\
\text { number }\end{array}$ & Effective theory & $\begin{array}{l}\text { Symmetry } \\
\text { requirement }\end{array}$ & Physical properties \\
\hline \multirow[t]{3}{*}{ Family 1} & $2+1$ & $1^{\text {st }}$ Chern number $C_{1} \in \mathbb{Z}$ & $\mathcal{L}=\frac{C_{1}}{2 \pi} A_{\mu} \epsilon^{\mu \nu \rho} \partial_{\nu} A_{\rho}$ & Not required & $\begin{array}{l}\text { QH effect; axial anomaly } \\
\text { on the boundary }\end{array}$ \\
\hline & $1+1$ & $\begin{array}{c}1^{\text {st }} \text { Chern parity } \\
N_{1}=(-1)^{C_{1}\left[h_{0}, h\right]} \in Z_{2}\end{array}$ & $\mathcal{L}=P_{1} \epsilon^{\mu \nu} \partial_{\mu} A_{\nu}$ & $\mathrm{C}$ & $\begin{array}{l}\text { Half charge on the } \\
\text { boundary }\end{array}$ \\
\hline & $0+1$ & $\begin{array}{c}1^{\text {st }} \text { Chern parity } \\
N_{0}=N_{1}\left[h_{0}, h\right] \in Z_{2}\end{array}$ & $\mathcal{L}=\operatorname{Tr}\left[a_{0}\right]$ & $\mathrm{C}$ & Not applicable \\
\hline \multirow[t]{3}{*}{ Family 2} & $4+1$ & $2^{\text {nd }}$ Chern number $C_{2} \in \mathbb{Z}$ & $\mathcal{L}=\frac{C_{2}}{24 \pi^{2}} A_{\mu} \epsilon^{\mu \nu \rho \sigma \tau} \partial_{\nu} A_{\rho} \partial_{\sigma} A_{\tau}$ & Not required & $\begin{array}{c}\text { 4DQH effect; chiral } \\
\text { anomaly on the boundary }\end{array}$ \\
\hline & $3+1$ & $\begin{array}{c}2^{\text {nd }} \text { Chern parity } \\
N_{3}=(-1)^{C_{2}\left[h_{0}, h\right]} \in Z_{2}\end{array}$ & $\mathcal{L}=\frac{1}{4 \pi} P_{3} \epsilon^{\mu \nu \sigma \tau} \partial_{\mu} A_{\nu} \partial_{\sigma} A_{\tau}$ & $\mathrm{T}$ & $\begin{array}{l}\text { Half QH effect on the } \\
\text { boundary, TME effect }\end{array}$ \\
\hline & $2+1$ & $\begin{array}{c}2^{\text {nd }} \text { Chern parity } \\
N_{2}=N_{3}\left[h_{0}, h\right] \in Z_{2}\end{array}$ & $\mathcal{L}=\frac{1}{2 \pi} A_{\mu} \epsilon^{\mu \nu \rho} \partial_{\nu} \Omega_{\rho}$ & $\mathrm{T}$ & $\begin{array}{l}\text { QSH effect; half charge at } \\
\text { anti-phase domain wall on } \\
\text { the boundary }\end{array}$ \\
\hline
\end{tabular}

TABLE I: Summary on the properties of the topological insulators. In the effective Lagrangians, the indices $\alpha, \beta$.. $=0,1$, $i, j . .=0,1,2, \mu, \nu_{. .}=0,1,2,3$ and $a, b . .=0,1, . ., 4$. The vector $a_{i}\left(a_{\mu}\right.$, et al.) stands for the gauge vector of the external electro-magnetic field, and $A_{i}$ that for the Berry phase gauge field. $C$ and $T$ stands for particle-hole symmetry and time-reversal symmetry, respectively. The quantities $P_{1}, P_{3}, \Omega_{\rho}$ are defined by Eqs. (24), (79) and (112). See text of Sec. VI for explanations of the effective actions.

gauge field $A_{\mu}$ depends only on the $(1+1)$-d coordinates $(t, x)$ after dimensional reduction. Consequently, in the Chern-Simons form $\epsilon^{\mu \nu \tau} A_{\mu} \partial_{\nu} A_{\tau}$ the terms containing $\partial_{y}$ are identically zero, so that

$\epsilon^{\mu \nu \tau} A_{\mu} \partial_{\nu} A_{\tau}=2\left(A_{t} \partial_{x} A_{y}-A_{x} \partial_{t} A_{y}\right)=2\left(A_{t} \partial_{x} \theta-A_{x} \partial_{t} \theta\right)$

after an integration by parts. The effective action (122) after making all four substitutions above is expressed as

$$
\begin{aligned}
S_{1+1}= & \frac{1}{2 \pi} \int d k_{x} \operatorname{Tr}\left[\partial_{k_{x}} a_{\theta}-\partial_{\theta} a_{k_{x}}\right] \\
& \cdot \int d t d x\left(A_{t} \partial_{x} \theta-A_{x} \partial_{t} \theta\right) .
\end{aligned}
$$

With a smooth space-time dependent $\theta(x, t)$ field, the eigenstates $\left|\alpha ; k_{x}, \theta\right\rangle$ can be considered as space-time dependent "local" eigenstates, whose space-time dependence originates only from that of $\theta(x, t)$. In this way, each state $\left|\alpha ; k_{x}, \theta(x, t)\right\rangle=\left|\alpha ; k_{x}, x, t\right\rangle$ is defined in the full phase space $\left(t, x, k_{x}\right)$, and the Berry's phase gauge field can gain real-space components defined as $a_{\mu}^{\alpha \beta}=$ $-i\left\langle\alpha k_{x}, x, t\left|\partial_{\mu}\right| \beta, k_{x}, x, t\right\rangle=a_{\theta} \partial_{\mu} \theta$, in which $\mu=t, x$. Similarly, the space-time derivative of $a_{k_{x}}$ is given by $\partial_{\mu} a_{k_{x}}=\partial_{\theta} a_{k_{x}} \partial_{\mu} \theta$. By making use of these observations, the effective action can be simplified to

$$
\begin{aligned}
S_{1+1}= & \frac{1}{2 \pi} \int d k_{x} \int d t d x\left\{A_{t} \operatorname{Tr}\left[\partial_{k_{x}} a_{x}-\partial_{x} a_{k_{x}}\right]\right. \\
& \left.-A_{x} \operatorname{Tr}\left[\partial_{k_{x}} a_{t}-\partial_{t} a_{k_{x}}\right]\right\} .
\end{aligned}
$$

By generalizing the definition of the gauge vector potential $\left(A_{t}, A_{x}\right)$ to the phase space vector $\left(A_{t}, A_{x}, 0\right)$, the equation above can be expressed as the mixed ChernSimons term in the phase space:

$$
S_{1+1}=\frac{1}{2 \pi} \int d^{3} q \epsilon^{A B C} A_{A} \operatorname{Tr}\left[\partial_{B} a_{C}\right]
$$

which describes an inhomogeneous $(1+1)$-d insulator. Note that momentum derivatives acting on $\left(A_{t}, A_{x}\right)$ vanish. This effective action agrees our discussion in Sec. IIC as one can see by taking $\frac{\delta S_{1+1}}{\delta A_{A}}$ and comparing the resulting response equations with Eq. (28).

It should be noted that such a phase space formalism is only applicable when the space-time variation of $\theta$ is smooth and can be approximated by a constant in the neighborhood of a space-time point $(t, x, y)$. More quantitatively, the characteristic frequency $\omega$ and wavevector $k$ of $\theta(x, y, t)$ should satisfy

$$
\hbar \omega, \hbar v k \ll E_{g},
$$

where $v$ is a typical velocity scale of the system. For example, in the lattice Dirac model (12), $v$ is the speed of light (which is normalized to be 1 in Eq. (12)). Under condition (126) the space-time variation of the $\theta$ field does not generate excitations across the gap, and the system can be viewed locally as a band insulator with a "local Hamiltonian" $h\left(k_{x} ; x, t\right)$.

Carrying out such a procedure once more to the action (125) one can obtain the $(0+1)$-d action. We will show the derivation explicitly. First one must take $a_{x}=0, \partial_{x} a_{k_{x}}=0$ in Eq. (124) since nothing can depend on the spatial $x$-coordinate after it is dimensionally reduced. Next we replace $A_{x}$ by the parameter $\phi(t)-\phi_{0}$, which leads to

$$
\begin{aligned}
S_{0+1} & =-\int d t\left(\phi-\phi_{0}\right) \operatorname{Tr}\left[\partial_{\phi} a_{t}-\partial_{t} a_{\phi}\right] \\
& =-\int d t\left(\partial_{t} \phi \tilde{a}_{\phi}+\tilde{a}_{t}\right) .
\end{aligned}
$$

where the integration $\int d x d k_{x} / 2 \pi$ has been removed, and $a_{t}, a_{k_{x}}$ in (1+1)-d are relabelled as $\tilde{a}_{t}, \tilde{a}_{\phi}$ for later convenience and to obtain the second equality, an integration 
by parts is carried out. It should be noted that $\partial_{\phi}$ comes from $\partial / \partial k_{y}$, which actually means $\partial / \partial \phi_{0}$ since it is $\phi_{0}$ that is replacing $k_{y}$. Compared to the $(2+1)$-d $\rightarrow(1+1)$ $\mathrm{d}$ dimensional reduction, the difference here is that the wavefunctions are, in general, already time-dependent in $(1+1)-\mathrm{d}$. This comes from the dimensional reduction from $(2+1)-\mathrm{d}$. Consequently, the Berry's phase gauge potential in the $(0+1)$-d system consists of two terms as shown below:

$$
\begin{aligned}
a_{t}^{\alpha \beta} & =-i\left\langle\alpha ; t, \phi(t)\left|\partial_{t}\right| \beta ; t, \phi(t)\right\rangle \\
& =-i\left\langle\alpha ; t, \phi\left|\left[\left(\frac{\partial}{\partial t}\right)_{\phi}+\frac{\partial \phi}{\partial t}\left(\frac{\partial}{\partial \phi}\right)_{t}\right]\right| \beta ; t, \phi\right\rangle \\
& =\tilde{a}_{t}+\partial_{t} \phi \tilde{a}_{\phi}
\end{aligned}
$$

in which $\left(\partial / \partial_{t}\right)_{\phi}$ means to take the $t$-derivative while keeping $\phi$ constant. Both of these terms are necessary for the correct topological response and similar terms (including spatially dependent ones) will be present in all higher dimensions when more than one reduction is carried out. Combining the two equations above we finally obtain

$$
S_{0+1}=\int d t \operatorname{Tr}\left[a_{t}\right]
$$

which has the form of a "zero-th" Chern-Simons term, and describes the " $(0+1)$-d insulator" discussed in Sec. IIE, i.e., a single-site fermion system. In this case the only gauge invariant quantity remaining is the Berry's phase the single-site system obtains during a closed path of adiabatic evolution.

For the second family of topological insulators we discussed, the effective theory (52) can be expressed in the following phase space form:

$$
\begin{aligned}
S_{4+1}= & \frac{1}{192 \pi^{4}} \int d^{9} q \epsilon^{A B C D E F G H I} A_{A} \partial_{B} A_{C} \partial_{D} A_{E} \\
& \cdot \operatorname{Tr}\left[D_{F} a_{G} D_{H} a_{I}\right]
\end{aligned}
$$

in which the covariant derivative $D_{B}=\partial_{B}+i a_{B}$ is introduced for the non-abelian Berry's phase gauge field. The dimensional reduction to $(3+1)$ - $\mathrm{d}$ can be performed similarly to the $(2+1)$-d case. Denoting the 4 -th spatial dimension as $w$, which is the dimension to be reduced, then any term with $\partial_{w}$ vanishes, and so does $a_{w}$. Consequently, the only non-vanishing terms in the effective action (128) are those with $A_{w}$, which now is replaced by the parameter $\theta(x, y, z, t)$. On the other hand, one of $F, G, H, I$ in the form $D_{F} a_{G} D_{H} a_{I}$ has to be $k_{w}$, which is now replaced by $\theta$. In summary the theory can be rewritten as

$$
\begin{aligned}
S_{3+1}= & \frac{3}{96 \pi^{3}} \int d^{7} q \epsilon^{\mu \nu \sigma \tau} \epsilon^{i j k} A_{\mu} \partial_{\nu} A_{\sigma} \partial_{\tau} \theta \\
& \cdot \operatorname{Tr}\left[D_{\theta} a_{i} D_{j} a_{k}+\text { cycl. }\right]
\end{aligned}
$$

where $\mu, \nu_{.}=0,1,2,3$ and $i, j, k=1,2,3$ are space-time and momentum indices of $(3+1)$-d system, and cycl. denotes the other three terms obtained from cyclicly permuting $\theta$ and $i, j, k$. The integration $\int d k_{w} d w / 2 \pi$ has been removed, and a prefactor 3 appears due to the fact that there are three $A_{A}$ 's in the effective action (128). In the same way as in $(2+1)$-d to $(1+1)$-d case, $\partial_{\mu} \theta D_{\theta}$ can be replaced by $D_{\mu}$, so that the effective theory of the $(3+1)$-d insulator is finally obtained:

$$
S_{3+1}=\int \frac{d^{7} q}{32 \pi^{3}} \epsilon^{A B . . G} A_{A} \partial_{B} A_{C} \operatorname{Tr}\left[D_{D} a_{E} D_{F} a_{G}\right] .
$$

According to the definition of $P_{3}$ in Eq. (779) we know that

$$
\partial_{\ell} P_{3}=\frac{1}{8 \pi^{2}} \int d^{3} k \epsilon^{\theta i j k} \operatorname{Tr}\left[f_{\ell i} f_{j k}\right],
$$

which shows the equivalence of the action (129) to the action (80) we derived earlier.

Now from the two examples of $(2+1)-d \rightarrow(1+1)$-d $\rightarrow(0+1)-\mathrm{d}$ and $(4+1)-\mathrm{d} \rightarrow(3+1)-\mathrm{d}$, one can easily obtain the general rule of dimensional reduction to the phase-space Chern-Simons theories. For a $(d+1)$ dimensional system, the phase space dimension is $2 d+1$, and the dimensional reduction of corresponding phase-space Chern-Simons theory is defined as

1. Remove a term $\partial_{A} A_{B}$ from the action and correspondingly replace the $(2 d+1)$-dimensional anti-symmetric tensor by the one in $(2 d-1)$ dimensions.

2. Remove the integration $\int d x_{d} d k_{d} / 2 \pi$ from the action when $x_{d}, k_{d}$ are the spatial and momentum indices to be reduced.

3. Multiply the action by a factor $n$ when the power of external gauge field $A_{A}$ in the original action is $n$.

Following these rules, the effective action for $(2+1)-\mathrm{d}$ TRI insulator can be easily obtained by one more step of dimensional reduction from Eq. (129):

$$
S_{2+1}=\int \frac{d^{5} q}{8 \pi^{2}} \epsilon^{A B C D E} A_{A} \operatorname{Tr}\left[D_{B} a_{C} D_{D} a_{E}\right]
$$

where the coefficient is determined by $8 \pi^{2}=32 \pi^{3} /(2$. $2 \pi)$. By considering the space-time and momentum indices separately, one can easily confirm that Eq. (130) is equivalent to Eq. (115) we obtained earlier.

In summary, we have shown that all the known topological insulators are described by a Chern-Simons effective theory in phase space, and the topological theories in different dimensions can be related by the dimensional reduction procedure. It is straightforward to generalize this formalism to arbitrary dimensions. As shown in Appendix $\mathrm{B}$, the relation between the non-linear response function (53) and the corresponding Chern number in momentum space can be generically proven for any odd space-time dimension. The effective theory of such a $(2 n+1)$-d topological insulator is given by 40 


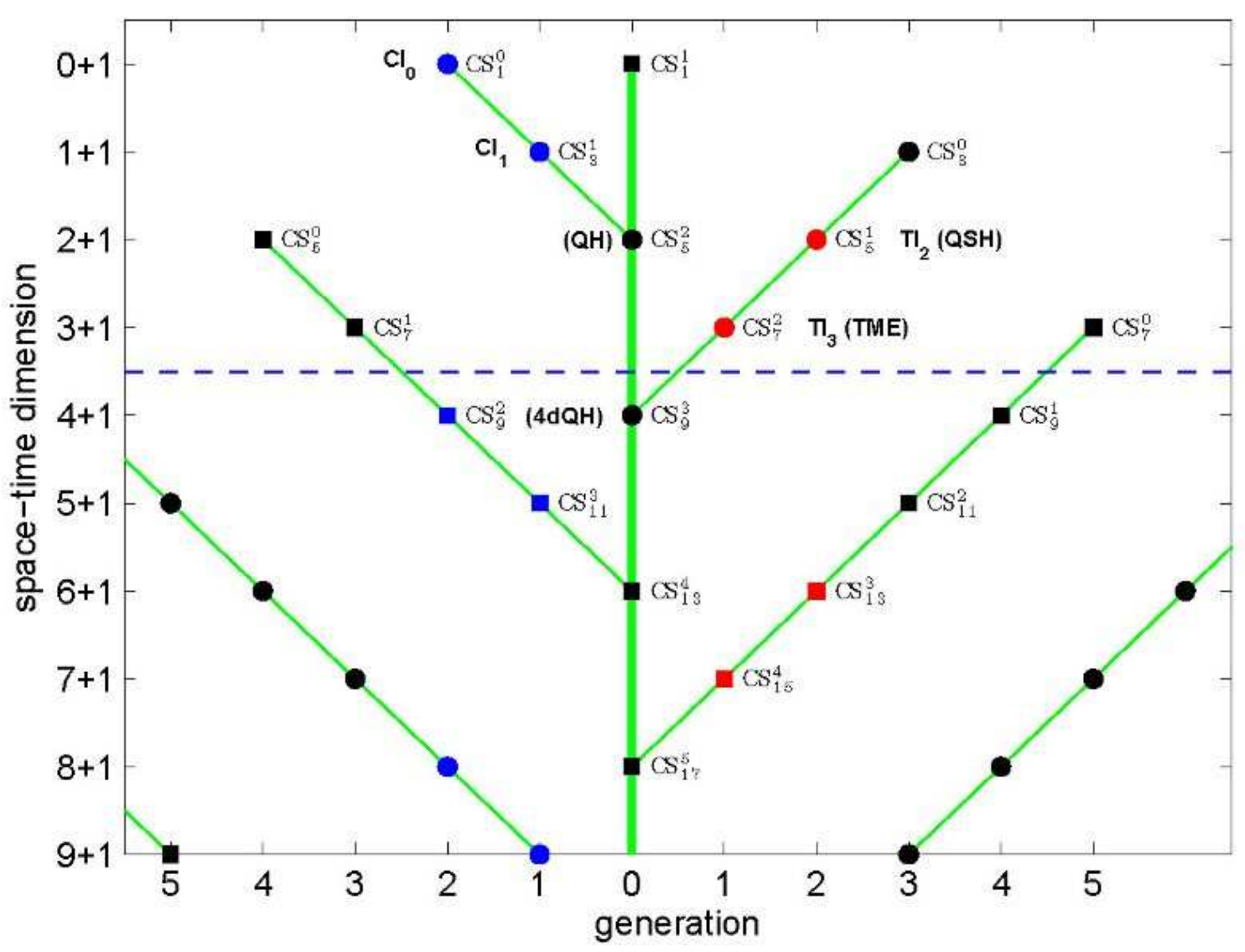

FIG. 21: The family tree of topological insulators. The black points on the "trunk" (i.e., 0-th generation) stand for the fundamental topological insulators in odd space-time dimensions characterized by a nontrivial Chern number in momentum space. The blue and red markers show the descendants of the $(4 n-1)$-d and $(4 n+1)$-d insulators, respectively. Physical effects associated with some of the prominent topological insulators are indicated in parenthesis. A $Z_{2}$ classification is defined for each blue circle (square) under (pseudo) particle-hole symmetry $C(\tilde{C})$, and for each red circle (square) under (pseudo) time-reversal symmetry $T(\tilde{T})$. The definitions of $C, \tilde{C}, T, \tilde{T}$ are given in Sec. VIB For all the physically realizable systems with spatial dimensions $d \leq 3$, the names of topological insulators are labelled, where $\mathrm{CI}_{\mathrm{n}}\left(\mathrm{TI}_{\mathrm{n}}\right)$ stands for a particle-hole symmetric (TRI) topological insulator in $n+1$ dimension. The black circles and squares stand for other topological phenomena obtained from dimensional reduction, which are also described by the phase space Chern-Simons theories but do not correspond to $Z_{2}$ topological insulators. The phase space Chern-Simons theory $\mathrm{CS}_{2 n+1}^{t}$ (as defined in Eq. (134)) corresponding to each topological phenomenon is also specified on the figure.

$$
S_{2 n+1}=\frac{C_{n}}{(n+1) !(2 \pi)^{n}} \int d^{2 n+1} x \epsilon^{\mu_{1} \mu_{2} . . \mu_{2 n+1}} A_{\mu_{1}} \partial_{\mu_{2}} A_{\mu_{3}} . \partial_{\mu_{2 n}} A_{\mu_{2 n+1}}
$$

with the $n$-th Chern number in momentum space defined as

$$
C_{n}=\frac{1}{n ! 2^{n}(2 \pi)^{n}} \int d^{2 n} k \epsilon^{i_{1} i_{2} . . i_{2 n}} \operatorname{Tr}\left[f_{i_{1} i_{2}} f_{i_{3} i_{4} . .} f_{i_{2 n-1} i_{2 n}}\right] .
$$

Thus the $(4 n+1)$-d phase space formula for this effective action can be written as

$$
S_{2 n+1}=\frac{1}{n !(n+1) !(2 \pi)^{2 n}} \int d^{4 n+1} q \epsilon^{A_{1} A_{2} \ldots A_{4 n+1}} A_{A_{1}} \partial_{A_{2}} A_{A_{3} . .} \partial_{A_{2 n}} A_{A_{2 n+1}} \operatorname{Tr}\left[D_{A_{2 n+2}} a_{\left.A_{2 n+3} . . D_{A_{4 n}} a_{A_{4 n+1}}\right]}\right.
$$

Following the general rules of dimensional reduction procedure discussed above, one can obtain the effective actions for lower-dimensional topological insulators as "descendants" of the topological theory (133). From the examples discussed above it can be seen that the number of Berry's phase gauge vectors $a_{i}$ in the effective action remains 
invariant during dimensional reduction, while the number of external gauge field insertions $A_{\mu}$ decreases by one at each step of dimensional reduction. After $n+1$ reductions we obtain an effective action of an $((n-1)+1)$-d system that contains no $A_{i}$. Just like in the $(0+1)$-d case, such an effective action does not result in any response equation of the system, but only describes the Berry's phase the system obtains during adiabatic evolution. Obviously the dimensional reduction cannot be carried out again on such an $((n-1)+1)$-d system. Thus, the $(2 n+1)$-d topological insulator with a nontrivial $n$-th Chern number only has $(n+1)$ "descendants" under dimensional reduction. It is straightforward to show that the $m$-th descendant $(1 \leq m \leq n+1)$ of the $(2 n+1)$-d topological insulator has the effective action

$$
\begin{aligned}
S_{2 n+1-m}^{(m)} & =\frac{\left(\begin{array}{l}
2 n+1-m \\
n
\end{array}\right)}{(2 n+1-m) !} \int \frac{d^{4 n+1-2 m} q}{(2 \pi)^{2 n-m}} \epsilon^{A_{1} A_{2} . . A_{4 n+1-2 m}} A_{A_{1}} \partial_{A_{2}} A_{A_{3} . . \partial_{A_{2 n-2 m}} A_{A_{2 n+1-2 m}}} \\
\cdot & \cdot \operatorname{Tr}\left[D_{A_{2 n+2-2 m}} a_{A_{2 n+3-2 m}} . . D_{A_{4 n-2 m}} a_{A_{4 n+1-2 m}}\right] \\
& \equiv \mathrm{CS}_{4 n-2 m+1}^{n-m+1}
\end{aligned}
$$

in which $\mathrm{CS}_{s}^{t}$ stands for the mixed Chern-Simons action in $s$ phase-space dimensions with $t$ powers of the external $A_{A}$ field. Specifically, $\mathrm{CS}_{s}^{0}$ is a pure non-Abelian Chern-Simons term of the Berry's phase gauge field $a_{A}$, which cannot be reduced to a function of $f_{A B}=D_{A} a_{B}-D_{B} a_{A}$ alone. Thus we have seen that a whole family of topological phenomena are described by phase-space Chern-Simons theories with different $s, t$ values. In a given spatial dimension $d$, all possible topological phenomena in band insulators are given by the actions $\operatorname{CS}_{2 d+1}^{t}$ with all possible values of $t$. It should be noted that the external gauge field $A_{A}$ is only defined in real space, meaning that $A_{A}$ or $\partial_{B} A_{A}$ are both vanishing if $A$ or $B$ is a momentum index $A, B=d+2, d+3, \ldots, 2 d+1$. Since in $\mathrm{CS}_{2 d+1}^{t}$ there are $t A_{A}$ 's and at least $t-1$ partial derivative operators acting on $A_{A}$ 's, the Chern-Simons action $\operatorname{CS}_{2 d+1}^{t}$ vanishes if $2 t-1>d+1$. Consequently, there are in total $[d / 2]+2$ available Chern-Simons terms in the phase space of a $(d+1)$-dimensional system, which are

$$
\mathrm{CS}_{2 d+1}^{t}, t=0,1, \ldots,[d / 2]+1 .
$$

Here $[d / 2]$ denotes the maximal integer that does not exceed $d / 2$. For example, in $(2+1)$-d there are three available phase space Chern-Simons terms, two of which are $\mathrm{CS}_{5}^{2}$ describing a QH insulator, and $\mathrm{CS}_{5}^{1}$ describing a QSH insulator. The third one is given by

$$
\begin{aligned}
\mathrm{CS}_{5}^{0}= & \frac{1}{3 !(2 \pi)^{2}} \int d^{5} q \epsilon^{A B C D E} \operatorname{Tr}\left[a_{A} \partial_{B} a_{C} \partial_{D} a_{E}\right. \\
& \left.+\frac{3}{2} a_{A} a_{B} a_{C} \partial_{D} a_{E}+\frac{3}{5} a_{A} a_{B} a_{C} a_{D} a_{E}\right]
\end{aligned}
$$

which contains no $A_{A}$ and thus does not describe any electromagnetic response properties of the system. The information contained in the effective action $\mathrm{CS}_{5}^{0}$ is the Berry's phase the system obtains during adiabatic evolution, just like the effective action of the $(0+1)$-d system $\mathrm{CS}_{1}^{0}=\int d t a_{0}$. We have grouped the phase-space Chern-Simons theories based on the parent theories and their descendants. The relationships are summarized in a "family-tree" in Fig. 21. Similar to the generalization of the $(2+1)$-d QH insulator to any odd space-time dimension, the $Z_{2}$ topological insulators we have studied can also be generalized to higher dimensions, which will be explained in the next subsection.

Before moving on to that, we would like to point out an interesting mathematical fact about the phase-space Chern-Simons theories. For an $(n+1)$ dimensional system with $N$ occupied bands, a $U(N)$ gauge vector potential can be defined in phase space as

$$
\mathcal{A}_{A}^{\lambda}=\lambda A_{A}+a_{A},
$$

with $A_{A}$ being the external gauge potential and $a_{A}$ being the Berry phase gauge potential. The non-Abelian ChernSimons term for $\mathcal{A}_{A}^{\lambda}$ can be expressed as

$$
\mathrm{CS}_{2 n+1}(\lambda) \equiv \mathrm{CS}_{2 n+1}\left[\mathcal{A}_{A}^{\lambda}\right]=\frac{1}{(n+1) !(2 \pi)^{n}} \int d^{2 n+1} q \epsilon^{A_{1} A_{2} \ldots A_{2 n+1}} \operatorname{Tr}\left[\mathcal{A}_{A_{1}}^{\lambda} \partial_{A_{2}} \mathcal{A}_{A_{3}}^{\lambda} \ldots \partial_{A_{2 n}} \mathcal{A}_{2 n+1}^{\lambda}+\mathrm{N} . \mathrm{T} .\right]
$$

Here N.T. stands for the non-Abelian terms containing

commutators of $\mathcal{A}_{A}^{\lambda}$, which can be determined by the 
relation of the $(2 d+1)$-d Chern-Simons form to the $((2 d+$ 1) +1$)$-d Chern form. For more details, c.f. Section 11.5 of Ref. 71. By expanding the parameterized action $\mathrm{CS}_{2 n+1}(\lambda)$ over $\lambda$, the following equality can be obtained:

$$
\begin{aligned}
\mathrm{CS}_{2 n+1}(\lambda) & =\sum_{t=0}^{[n / 2]+1} \lambda^{t} \mathrm{CS}_{2 n+1}^{t} \\
\Rightarrow \mathrm{CS}_{2 n+1}^{t} & =\left.\frac{1}{t !} \frac{\partial^{t}}{\partial \lambda^{t}} \operatorname{CS}_{2 n+1}(\lambda)\right|_{\lambda=0} .
\end{aligned}
$$

This implies that all possible phase space Chern-Simons terms can be obtained from a single "generating functional" $\mathrm{CS}_{2 n+1}(\lambda)$. We present (135) as the unified theory of all topological insulators.

\section{B. $Z_{2}$ topological insulator in generic dimensions}

For the descendants of the $(2+1)$-d and $(4+1)$-d insulators, we have defined a $Z_{2}$ classification under the constraint of a discrete symmetry. For the descendants of the $(2+1)$-d QH insulator, the $Z_{2}$ classification is defined for particle-hole symmetric insulators satisfying Eq. (32):

$$
C^{\dagger} h(-\mathbf{k}) C=-h^{T}(\mathbf{k}), C^{\dagger} C=C^{*} C=\mathbb{I} .
$$

The key point of this classification is to show that an interpolation between two particle-hole symmetric Hamiltonians $h_{1}(k)$ and $h_{2}(k)$ forms a closed path when combined with its particle-hole transformed partner. The Chern number enclosed in such a closed path always has a certain parity which does not depend on the choice of the path. In the same way, a $Z_{2}$ classification of particlehole symmetric insulators is also defined in $(0+1)$-d. For the family of $(4+1)$-d insulators it is the same story except that the particle-hole symmetry is replaced by time-reversal symmetry

$$
T^{\dagger} h(-\mathbf{k}) T=h^{T}(\mathbf{k}), T^{\dagger} T=-T^{*} T=\mathbb{I} .
$$

Following this one can easily generalize such $Z_{2}$ classifications to higher dimensions. To do that, one first needs to understand what is the difference between $(2+1)$ and $(4+1)$ dimensions that requires the choice of different discrete symmetries. The easiest way to see such a difference is to study the transformation of the corresponding Chern-Simons theories under particle-hole symmetry $(C)$ and time-reversal symmetry $(T)$. Under particlehole symmetry, the charge density and charge current both change sign. The vector potential does as well, as required by the invariance of the minimal coupling $A_{\mu} j^{\mu}$. In the same way one can obtain the time-reversal property of $A_{\mu}$, as summarized below:

$$
C: A_{\mu} \rightarrow-A_{\mu}, T: A_{\mu} \rightarrow\left\{\begin{array}{c}
A_{0} \\
-A_{i}
\end{array}\right.
$$

In both cases of $C$ and $T$, the momentum operator $-i \partial_{\mu}$ has the same transformation property as $A_{\mu}$. Based on these facts the transformation properties of the ChernSimons Lagrangian (131) are

$$
\begin{aligned}
& C: S_{2 n+1}^{\mathrm{CS}} \rightarrow(-1)^{n+1} S_{2 n+1}^{\mathrm{CS}} \\
& T: S_{2 n+1}^{\mathrm{CS}} \rightarrow(-1)^{n} S_{2 n+1}^{\mathrm{CS}} .
\end{aligned}
$$

Thus, we see that $S_{4 n+1}^{\mathrm{CS}}$ is $T$-even but $C$-odd, while $S_{4 n-1}^{\mathrm{CS}}$ is $T$-odd but $C$-even. In other words, a $(4 n+1)$-d topological insulator has to break particle-hole symmetry but can be time-reversal invariant, just like the case of $(4+1)-\mathrm{d}$; a $[(4 n-2)+1]-\mathrm{d}$ topological insulator has to break time-reversal symmetry but can be particle-hole symmetric, just like the case of $(2+1)$-d. Consequently, for the descendants of $(4 n+1)-\mathrm{d}((4 n-1)-d)$ topological insulators, it is only possible to define $Z_{2}$ topological classifications by the dimensional reduction procedure under the constraint of $T(C)$ symmetry.

Naively, it seems that the procedure we introduced to define the $Z_{2}$ classification by dimensional reduction could be applied recursively to all the descendants of a $(2 n+1)$-d topological insulator. However, this turns out to be incorrect. As an example, we can study the $(1+1)$ d TRI insulator as a descendant of the $(2+1)$-d QSH insulator(not the $(2+1)$-d QH insulator). In the dimensional reduction from $(3+1)$-d to $(2+1)$-d discussed in Sec. VB. we define an interpolation $h(\mathbf{k}, \theta)$ between two $(2+1)$-d TRI Hamiltonians $h_{1}(\mathbf{k})$ and $h_{2}(\mathbf{k})$. When the interpolation $h(\mathbf{k}, \theta)$ is required to satisfy time-reversal symmetry (Eq. (119)), it corresponds to the Hamiltonian of a $(3+1)$-d topological insulator, for which a $Z_{2}$ index $N_{3}[h(\mathbf{k}, \theta)]$ can be defined. In Sec. VB we have shown that $N_{3}[h(\mathbf{k}, \theta)]$ does not depend on the choice of the interpolation $h(\mathbf{k}, \theta)$, which thus provides a criteria on whether $h_{1}(\mathbf{k})$ and $h_{2}(\mathbf{k})$ are topologically equivalent. If we carry out the same procedure on $(1+1)$-d TRI insulators, it seems that for two Hamiltonians $h_{1}(k)$ and $h_{2}(k)$ a $Z_{2}$ topological classification can be defined in the same way. To see if this is true, one can again take the lattice Dirac model as an example. The single-particle Hamiltonian of $(2+1)$-d $4 \times 4$ lattice Dirac model with time-reversal symmetry is written as

$$
\begin{aligned}
h_{2 \mathrm{D}}(\mathbf{k})= & \Gamma^{1} \sin k_{x}+\Gamma^{2} \sin k_{y} \\
& +\Gamma^{0}\left[m+c\left(\cos k_{x}+\cos k_{y}\right)\right]
\end{aligned}
$$

which is in the topological nontrivial phase for $0<m<$ $2|c|$ or $-2|c|<m<0$. By dimensional reduction, $h_{2 \mathrm{D}}(\mathbf{k})$ can be considered as the interpolation between two $(1+1)$-d TRI Hamiltonians $h_{1}(k)=h_{2 \mathrm{D}}(k, 0)$ and $h_{2}(k)=h_{2 \mathrm{D}}(k, \pi)$. Thus if the $Z_{2}$ classification procedure applied to $(1+1)$-d systems, $h_{1}(k)$ and $h_{2}(k)$ should be topologically distinct when $h_{2 \mathrm{D}}(\mathbf{k})$ is in the nontrivial phase. In other words, it should be impossible to define another interpolation $h_{0}(k, \theta)$ between $h_{1}(k)$ and $h_{2}(k)$, which satisfies $T^{\dagger} h_{0}(k, \theta) T=h_{0}^{*}(-k,-\theta)=$ $h_{0}^{*}(-k, \theta), \forall \theta$. However, such an interpolation can in fact 
be constructed as follows:

$$
\begin{aligned}
h_{0}(k, \theta)= & \Gamma^{1} \sin k+\Gamma^{02} \sin ^{2} \theta \\
& +\Gamma^{0}\left(m+c \cos k_{x}+c \cos \theta\right)
\end{aligned}
$$

in which $\Gamma^{02}=i \Gamma^{0} \Gamma^{2}$ is even under time-reversal. The existence of two topologically distinct interpolations $h_{0}(k, \theta)$ and $h_{2 \mathrm{D}}(k, \theta)$ shows that it is not possible to define a $Z_{2}$ classification of $(1+1)$-d TRI insulators in the same way as in $(2+1)$-d and $(3+1)$-d. The main reason for the failure is that the proof in Sec. (VB) requires the parameterized Hamiltonian manifold to be simply connected. In other words, an interpolation $h(\mathbf{k}, \theta, \varphi)$ can always be defined for two interpolations $h(\mathbf{k}, \theta)$ and $h^{\prime}(\mathbf{k}, \theta)$. Similar arguments do not work in the classification of $(1+1)$-d Hamiltonians because it may not be possible to adiabatically deform one path to the other. In the example of the lattice Dirac model, the paths $h_{2 \mathrm{D}}$ and $h_{0}$ cannot be adiabatically connected, because the combined path

$$
g(k, \theta)=\left\{\begin{array}{cc}
h_{2 \mathrm{D}}(k, \theta), & \theta \in[0, \pi] \\
h_{0}(k, 2 \pi-\theta), & \theta \in[\pi, 2 \pi]
\end{array}\right.
$$

is a $(2+1)$-d Hamiltonian that breaks time-reversal symmetry and has a nontrivial first Chern number $C_{1}=-1$. Consequently, the path $g$ cannot be contracted to a point, and the definition of a path-independent $Z_{2}$ invariant fails.

From this example we have seen that the definition of a $Z_{2}$ topological classification for the descendants of $(2 n+1)$-d topological insulators fails when the dimension is reduced to $((2 n-3)+1)-\mathrm{d}$, since the lower Chern number $C_{n-1}$ is defined for each closed path of $((2 n-3)+1)$-d Hamiltonians, thus obstructing the adiabatic connection between two different paths. In other words, the $Z_{2}$ topological insulators as descendants of $(2 n+1)$-d topological insulators can only be defined in $((2 n-1)+1)$ and $((2 n-2)+1)-d$. Since a bulk topological insulator always corresponds to a topologically protected gapless edge theory, the validity of a $Z_{2}$ classification can also be justified by studying the stability of edge theories. As discussed in Sec IIIB the boundary theory of a $(4+1)$-d topological insulator with second Chern number $C_{2}$ contains of $\left|C_{2}\right|$ flavors of chiral (Weyl) fermions. In the simplest example of the lattice Dirac model (61) with $-4 c<m<-2 c$, the boundary single-particle Hamiltonian is

$$
H_{\partial(4+1)}=v \vec{\sigma} \cdot \overrightarrow{\mathbf{p}}
$$

which is topologically stable since no mass term is available for the edge system. Under dimensional reduction the boundary theory of a $(3+1)-\mathrm{d} Z_{2}$ nontrivial insulator is simply given by taking $p_{z}=0$ in the above equation:

$$
H_{\partial(3+1)}=v\left(\sigma_{x} p_{x}+\sigma_{y} p_{y}\right)
$$

which is stable in the presence of time-reversal symmetry since no T-invariant mass terms are available. The same analysis shows the stability of the edge theory of a $(2+1)$ $\mathrm{d}$ topological insulator, given by $H_{\partial(2+1)}=v \sigma_{z} p_{z}$. When dimensional reduction is carried out once more, we obtain the $(0+1)$ - $d$ edge of the $(1+1)$-d insulator described by $H_{\partial(1+1)}=0$. This just describes a Kramers's pair of localized states on the boundary. Since such a pair of mid-gap states can be easily removed by a constant energy shift without breaking time-reversal symmetry, the $(1+1)$-d TRI insulator does not have a topologically nontrivial class. Different edge state stabilities for effective theories in different dimensions are illustrated in Fig. 21.

Such an edge theory analysis can be easily generalized to higher dimensions. The boundary states of a $(2 n+1)$ $\mathrm{d}$ topological insulator with nontrivial Chern number are described by a $((2 n-1)+1)$-d chiral fermion theory with the Hamiltonian

$$
H_{2 n-1}(\mathbf{p})=v \sum_{i=1}^{2 n-1} p_{i} \Gamma^{i}
$$

in which $\Gamma^{i}$ are $2^{n-1} \times 2^{n-1}$ matrices forming a representation of the $s o(2 n-1)$ Clifford algebra. The boundary theory of the $m$-th descendant of the $(2 n+1)$-d system is given by simply taking $p_{i}=0$ for $i=2 n-m, 2 n-$ $m+1, \ldots, 2 n-1$. The symmetry properties and stability of the theory can be studied by studying the properties of the $\Gamma^{a}$ matrices. Here we will display the conclusions of the edge state analysis, with the details presented in Appendix D.

1. The chiral Hamiltonians (143) in different dimensions satisfy different discrete symmetry properties, as listed below:

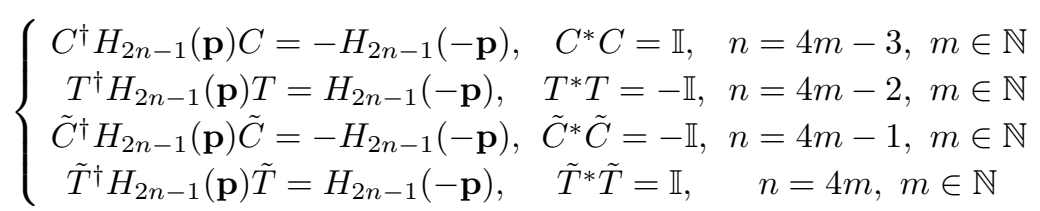

2. Only the first two descendants of $H_{2 n-1}$, i.e., $H(\mathbf{p})=\sum_{i=1}^{2 n-2} p_{i} \Gamma^{i}$ and $H(\mathbf{p})=\sum_{i=1}^{2 n-3} p_{i} \Gamma^{i}$, are topologically stable under the constraint of the discrete symmetry in the given dimension $(C, \tilde{C}, T$ or $\tilde{T})$. Consequently, the $Z_{2}$ topologically nontrivial insulators descending from the $(2 n+1)$-d topological insulator only exist in $((2 n-1)+1)$-d and $((2 n-2)+1)$-d. 
The edge state analysis confirms our insight from the bulk picture, that is, the $Z_{2}$ topological classification is only well defined for the first two generations of descendants of the $(2 n+1)$-d topological insulator. Moreover, it also provides more information about the discrete symmetries in different dimensions. In the $(6+1)$-d topological insulator, and its descendants, the correct discrete symmetry is $\tilde{C}$ which is similar to particle-hole symmetry $C$ but satisfies $\tilde{C}^{*} \tilde{C}=-\mathbb{I}$. This is necessary since a usual particle-hole symmetry cannot be defined for the $(5+1)$ d chiral fermion $\sum_{i=1}^{5} p_{i} \Gamma^{i}$. Such a symmetry $\tilde{C}$ can be called a "pseudo particle-hole symmetry". Similarly, in the $(8+1)$-d topological insulator, and its descendants, the discrete symmetry is a "pseudo time-reversal symmetry" satisfying $T^{*} T=\mathbb{I}$. In Fig. 21] the dimensions with "true" $C$ or $T$ symmetry are labelled with filled circles, and those with $\tilde{C}$ or $\tilde{T}$ symmetry are labelled with squares.

This paper is partly inspired by work in high-energy physics. The study of topological insulators with nontrivial boundary states is analogous to the generation of massless fermions on higher dimensional domain wall $\$ 3$.72. In a high energy context these surface states would be subsequently used to mimic chiral fermions in lattice gauge theories. In our case they become the gapless boundary liquids that generate novel transport properties and characterize the topological stability of the state. Our picture of a $(4+1)$-d topological insulator characterized by the Chern-Simons term has a very special meaning in high-energy physics when reduced to $(3+1)$-d. The Chern-Simons term becomes a $\theta$-term which is related to the so-called vacuum angle. If $\theta$ is a constant then this term does not contribute to the equations of motion. In our case the $\theta$-term has a solitonic structure with a domain wall at the surface of the topological insulator. Inside the insulator $\theta$ jumps by $\pi$ which still preserves $\mathrm{CP}$ if the original vacuum does. Thus, the only effect on the system is a non-zero boundary term at the $\theta$ domain wall. From a high-energy perspective we have introduced an axionic domain wall and a topological insulator exists in one domain while a trivial insulator exists in the other.

The dimensional reduction procedure we introduced here is not new to physics, and was first used in the 1920's in an attempt to unify gravity and electro-magnetism in $(4+1)$-d by Kaluza and Klein ${ }^{73,74}$. Basically, the dimensional reduction amounts to compactifying the "extra dimension" with periodic boundary conditions, e.g. on a circle, and shrinking its radius to zero. The compactification creates a tower of modes labelled by a discrete index, but as the circle is shrunk only one low-energy mode from each field remains (which can be seen by taking the Fourier transform of a field on a circle with periodic boundary conditions). These "zero-modes" become the propagating fields in the lower dimensional space. Carrying out this procedure via our method or by compactification yields the same results. The adiabatic parameter we introduced is connected with the flux threading the higher dimensional circle. One could imagine compactifying using higher dimensional manifolds, such as a sphere or something more exotic, and threading various fluxes through non-trivial cycles of the compact space. The zero-mode structures of these manifolds are more complicated and we will not deal with them here, but perhaps other interesting theories can arise.

Additionally, we have unearthed a ladder of topological insulators with gapless fermionic boundary states whose stability depends on the presence of a discrete symmetry. The discrete symmetries repeat modulo 8 , which should be no surprise since their form is derived from the representation theory of real Clifford algebras which exhibit Bott periodicity with period $8^{75}$. Due to this periodicity we can analyze the types of gapless fermions allowed to exist at the boundaries of topological insulators. Any boundary can support Dirac fermions and any even dimensional space-time boundary can have Weyl (chiral) fermions as well. For Majorana fermions we are restricted to boundaries with spacetime dimensions $\{(1+1),(2+1),(3+1),(7+1),(8+1)\} \bmod 8$. Finally, there is a special representation, the MajoranaWeyl fermion, which is a real fermion with a definite handedness which can only exist in $(1+1) \bmod 8$ dimensions i.e. $(1+1),(9+1),(17+1),(25+1) \ldots \stackrel{75}{.}$. It is this type of fermion which appears at the edge of $(p+i p)$ superconductors $\frac{76,77,78}{}$. However, because this representation is missing in (5+1)-d a $(6+1)$-d " $(\mathrm{p}+\mathrm{ip})$ superconductor" which obeys the "pseudo-particle hole" symmetry will not have single Majorana-Weyl boundary states. This can also be seen by the fact that $\tilde{C}^{2}=-1$ which means there would have to be at the minimum two Majorana-Weyl fermions at the boundary due to a Kramers'-like theorem. Beginning with the parent $(2 n+1)-d$ topological insulator we see that the boundary theories of itself and its stable descendants are massless fermions in $((2 n-1)+1),((2 n-2)+1)$ and $((2 n-3)+1)$ dimensions respectively. Theories of massless fermions often result in field theory anomalies and there is a deep connection between this boundary theory ladder and the corresponding anomaly ladder $\underline{71,79}$.

In summary, we have provided a unified framework to describe a whole family of topological insulators in generic dimensions. All the topological effects are described by phase-space Chern-Simons theories, which either describe the topological insulators with nontrivial Chern number in odd space-time dimensions, or describe their lower dimensional descendants through dimensional reduction. $Z_{2}$ topologically nontrivial insulators exist in $((2 n-1)+1)$ and $((2 n-2)+1)$ space-time dimensions and are protected by a given discrete symmetry that is preserved by the parent $(2 n+1)$-d topological insulator. We found that in $(2 n+1)$-d there are two types of topological insulators, one of which is characterized by the Chern number $C_{n}$ and the other by the $Z_{2}$ invariant, as a descendant of $((2 n+2)+1)$-d topological insulator. In comparison, in $((2 n-1)+1)$-d there is only one type of topological insulator, which is characterized by a $Z_{2}$ 
invariant, as a descendant of $(2 n+1)$-d topological insulator. There are many tantalizing connections of our work with well-developed sectors of high-energy physics, and diving deeper into these subjects is sure to benefit both condensed-matter and high-energy physics.

\section{CONCLUSION AND DISCUSSIONS}

In conclusion we have constructed the topological field theory of TRI insulators. We showed that the fundamental TRI insulator naturally exists in $4+1-\mathrm{d}$, and the effective topological field theory is the Chern-Simons theory in $4+1$-d. We introduced the concept of dimensional reduction for microscopic fermion models, where some spatial dimensions are compactified and the associated momentum variables are replaced by adiabatic fields. This method enables us to obtain the topological field theory for the 3D and 2D TRI insulators from the dimensional reduction of the $4+1$-d Chern-Simons field theory. In particular, we obtain the "axion" field theory for the 3D insulator, and many experimental consequences follow directly from this topological field theory. The most striking prediction is the TME effect, where an electric field induces a magnetic field along the same direction, with a universal constant of proportionality quantized in odd multiples of the fine structure constant. The role of the "axion", or the adiabatic field, is played by a magnetoelectric polarization, whose change is quantized when an adiabatic process is completed. The topological field theory for the 2D TRI insulator involves two adiabatic fields, and this theory directly predicts the fractional charge of a magnetic domain wall at the edge of the QSH insulator. These topological effects illustrate the predictive power of the topological field theory constructed in this work, and many more experimental consequences can be obtained by the proper generalization of the concepts introduced here.

Our work also presents the general classification of topological insulators in various dimensions. The fundamental topological insulators are described by the topological Chern-Simons field theory, and the effective topological field theory of their descendants can be obtained by the procedure of dimensional reduction. The descendent topological insulators are generally classified by discrete symmetries like the charge conjugation and the time reversal symmetries. This way, the Chern number classification of fundamental topological insulators and the $Z_{2}$ classifications of their descendants is unified. Finally, we present a framework in terms of the Chern-Simons field theory in phase space, which gives a unified theory of all topological insulators and contains all experimentally observable topological effects.

We would like to thank Dr. B. A. Bernevig for many insightful discussions on this subject and collaborations at the early stage of this project. We would like to thank H. D. Chen, S. Kachru, C. X. Liu, J. Maciejko, M. Mulligan, S. Raghu, S. Shenker and J. Zaanen for helpful discussions. This work is supported by the NSF under grant numbers DMR-0342832 and the US Department of Energy, Office of Basic Energy Sciences under contract DE-AC03-76SF00515.

\section{APPENDIX A: CONVENTIONS}

Due to the special importance of dimensionality in this work we tried to be very consistent with our dimension and index conventions. In addition, gauge fields of all types appear and we have selected a convention for the electro-magnetic vector potential and the adiabatic (Berry's phase) connection. The conventions we used in this paper are summarized in Table $\amalg$ and also explained below.

For spacetime conventions we have chosen the form $(n+1)$-d where $n$ can be 0 . For spatial dimensions only we use $\mathrm{nD}$ with a capital $\mathrm{D}$.

For indices, space-time and frequency-momentum indices share the same convention. Greek indices from the middle of the alphabet such as $\mu, \nu, \rho, \sigma, \tau$ run from 0 to the spatial dimension in the current context. Examples being $0,1,0,1,2$ etc. Additionally, when indexing momentum space objects 0 is frequency and $1,2,3 \ldots$ are the momentum components in the $1,2,3, \ldots$ directions. Latin indices from the middle of the alphabet such as $i, j, k, \ell$ are purely spatial indices and run from 1 onward to the spatial dimension of the current context. For momentum space objects they index the spatial momenta e.g $k_{x}, k_{y}, k_{z}, k_{w} \ldots$ We always use the Einstein summation convention unless stated otherwise. Since we are considering flat space we make no distinction between raised and lowered indices.

In some special contexts we will need two more sets of indices. Several of the Hamiltonian models we use can be written in terms of the $2 \times 2$ or $4 \times 4$ Dirac matrices. In these cases where you see vectors $d^{a}$ indexed by lowercase Latin letters from the beginning of the alphabet they run from 1,2,3 and 0,1,2,3,4 respectively. Finally, for cases where the indices don't just run over coordinate or momentum space separately, but instead cover "phase-space" coordinates, we use capital Latin letters from the beginning of the alphabet such as $A, B, C, D, E$. These run over the phase space variables in the current context. Some examples being $q^{A}=\left(t, x, y, k_{x}, k_{y}\right)$, $q^{A}=\left(t, x, k_{x}\right)$, or A running over $\left(k_{x}, k_{y}, \theta, \phi\right)$.

For orbital, band, or state labels we use Greek letters from the beginning of the alphabet such as $\alpha, \beta, \gamma$.

For the electro-magnetic $U(1)$ gauge field we use $A^{\mu}$ where $A$ is capitalized. For the Berry's phase gauge field we use $a^{i}$ with a lower case $a$. Note the different index labels. The electromagnetic gauge field has a 0-component while the Berry's phase has no "frequency"-component. For the curvatures we use $F_{\mu \nu}$ and $f_{i j}$ respectively. 


\begin{tabular}{l|r}
\hline symbol & explanation \\
\hline$(n+1)$-d & space-time dimension $(n+1)$ \\
$n \mathrm{D}$ & spatial dimension $n$ \\
$\mu, \nu, \rho, \sigma, \ldots$ & space-time or frequency-momentum indices $0,1, \ldots, n$ \\
$i, j, k, l, \ldots$ & spatial or momentum indices $1,2, \ldots, n$ \\
$A, B, C, D, \ldots$ & phase space indices $0,1, \ldots n, n+1, . .2 n+1$ \\
$a, b, c, d, \ldots$ & indices for anticommuting $\Gamma^{a}$ matrices and corresponding coefficients $d_{a}$ \\
$\alpha, \beta, \gamma, \delta \ldots$ & energy band indices \\
$A_{\mu}, F_{\mu \nu}$ & gauge vector potential and gauge curvature of external electro-magnetic field \\
$a_{i}, f_{i j}$ & (generally non-Abelian) Berry phase gauge vector potential and gauge curvature in momentum space \\
$A_{A}, a_{A}$ & electro-magnetic or Berry phase gauge vector potential defined in phase space, respectively \\
\hline
\end{tabular}

TABLE II: Table of conventions used in the paper.

\section{APPENDIX B: DERIVATION OF EQ. (54)}

In this appendix we will prove the conclusion (54). As is briefly sketched in Sec. IIIA, the demonstration consists of three steps: (1) Topological invariance of Eq. (53); (2) Any Hamiltonian $h(\mathbf{k})$ is adiabatically connected to an $h_{0}(\mathbf{k})$ with the form of Eq. (55); (3) For such an $h_{0}(\mathbf{k})$ the non-linear correlation function (53) is equal to the second Chern number. In the following we will demonstrate these three steps separately.

\section{Topological invariance of Eq. (53)}

To prove the topological invariance of Eq. (53), we just need to prove any infinitesimal deformation of the Green's function $G(\mathbf{k}, \omega)$ leads to a vanishing variation of $C_{2}$. Under a variation of $G(\mathbf{k}, \omega)$ we have

$$
\begin{aligned}
\delta\left(G \partial_{\mu} G^{-1}\right) & =\delta G \partial_{\mu} G^{-1}+G \partial_{\mu}\left(\delta G^{-1}\right)=\delta G \partial_{\mu} G^{-1}-G \partial_{\mu}\left(G^{-1} \delta G G^{-1}\right) \\
& =-G\left(\partial_{\mu} G^{-1}\right) \delta G G^{-1}-\partial_{\mu}(\delta G) G^{-1}
\end{aligned}
$$

Thus the variation of $C_{2}$ is

$$
\begin{aligned}
\delta C_{2}= & -\frac{\pi^{2}}{15} \epsilon^{\mu \nu \rho \sigma \tau} \int \frac{d^{4} k d \omega}{(2 \pi)^{5}} \operatorname{tr}\left[\delta\left(G \partial_{\mu} G^{-1}\right)\left(G \partial_{\nu} G^{-1}\right)\left(G \partial_{\rho} G^{-1}\right)\left(G \partial_{\sigma} G^{-1}\right)\left(G \partial_{\tau} G^{-1}\right)\right] \\
= & \frac{\pi^{2}}{15} \epsilon^{\mu \nu \rho \sigma \tau} \int \frac{d^{4} k d \omega}{(2 \pi)^{5}} \operatorname{tr}\left[\left(G \partial_{\mu} G^{-1} \delta G G^{-1}\right)\left(G \partial_{\nu} G^{-1}\right)\left(G \partial_{\rho} G^{-1}\right)\left(G \partial_{\sigma} G^{-1}\right)\left(G \partial_{\tau} G^{-1}\right)\right] \\
& +\frac{\pi^{2}}{15} \epsilon^{\mu \nu \rho \sigma \tau} \int \frac{d^{4} k d \omega}{(2 \pi)^{5}} \operatorname{tr}\left[\left(\partial_{\mu} \delta G G^{-1}\right)\left(G \partial_{\nu} G^{-1}\right)\left(G \partial_{\rho} G^{-1}\right)\left(G \partial_{\sigma} G^{-1}\right)\left(G \partial_{\tau} G^{-1}\right)\right] \\
= & \frac{\pi^{2}}{15} \epsilon^{\mu \nu \rho \sigma \tau} \int \frac{d^{4} k d \omega}{(2 \pi)^{5}} \operatorname{tr}\left[\partial_{\mu}\left(G^{-1} \delta G\right)\left(\partial_{\nu} G^{-1} G\right)\left(\partial_{\rho} G^{-1} G\right)\left(\partial_{\sigma} G^{-1} G\right)\left(\partial_{\tau} G^{-1} G\right)\right] \\
= & \frac{\pi^{2}}{15} \epsilon^{\mu \nu \rho \sigma \tau} \int \frac{d^{4} k d \omega}{(2 \pi)^{5}} \partial_{\mu} \operatorname{tr}\left[\left(G^{-1} \delta G\right)\left(\partial_{\nu} G^{-1} G\right)\left(\partial_{\rho} G^{-1} G\right)\left(\partial_{\sigma} G^{-1} G\right)\left(\partial_{\tau} G^{-1} G\right)\right] \\
& -\frac{\pi^{2}}{15} \epsilon^{\mu \nu \rho \sigma \tau} \int \frac{d^{4} k d \omega}{(2 \pi)^{5}}\left\{\operatorname{tr}\left[\left(G^{-1} \delta G\right) \partial_{\mu}\left(\partial_{\nu} G^{-1} G\right)\left(\partial_{\rho} G^{-1} G\right)\left(\partial_{\sigma} G^{-1} G\right)\left(\partial_{\tau} G^{-1} G\right)\right]\right. \\
& +\operatorname{tr}\left[\left(G^{-1} \delta G\right)\left(\partial_{\nu} G^{-1} G\right) \partial_{\mu}\left(\partial_{\rho} G^{-1} G\right)\left(\partial_{\sigma} G^{-1} G\right)\left(\partial_{\tau} G^{-1} G\right)\right] \\
& +\operatorname{tr}\left[\left(G^{-1} \delta G\right)\left(\partial_{\nu} G^{-1} G\right)\left(\partial_{\rho} G^{-1} G\right) \partial_{\mu}\left(\partial_{\sigma} G^{-1} G\right)\left(\partial_{\tau} G^{-1} G\right)\right] \\
& \left.+\operatorname{tr}\left[\left(G^{-1} \delta G\right)\left(\partial_{\nu} G^{-1} G\right)\left(\partial_{\rho} G^{-1} G\right)\left(\partial_{\sigma} G^{-1} G\right) \partial_{\mu}\left(\partial_{\tau} G^{-1} G\right)\right]\right\} \\
= & \frac{\pi^{2}}{15} \epsilon^{\mu \nu \rho \sigma \tau} \int \frac{d^{4} k d \omega}{(2 \pi)^{5}} \partial_{\mu} \operatorname{tr}\left[\left(G^{-1} \delta G\right)\left(\partial_{\nu} G^{-1} G\right)\left(\partial_{\rho} G^{-1} G\right)\left(\partial_{\sigma} G^{-1} G\right)\left(\partial_{\tau} G^{-1} G\right)\right] \\
\equiv & 0 .
\end{aligned}
$$


Thus Eq. (53) is topologically invariant. The topological invariance of the second Chern number defined in Eq. (54) is a well-known mathematical fact. Such a topological invariance is quite helpful for showing the equivalence between the second Chern number and Eq. (53).

\section{Adiabatic deformation of arbitrary $h(\mathbf{k})$ to $h_{0}(\mathbf{k})$}

An adiabatic deformation $h(k, t), t \in[0,1]$ can be written down, which connects an arbitrary gapped Hamiltonian $h(\mathbf{k})$ to a "maximally degenerate Hamiltonian" $h_{0}(\mathbf{k})$ in the form of Eq. (55). Any single particle Hamiltonian $h(\mathbf{k})$ can be diagonalized as

$$
h(\mathbf{k})=U(\mathbf{k}) D(\mathbf{k}) U^{\dagger}(\mathbf{k})
$$

with $U(\mathbf{k})$ unitary and $h_{0}(\mathbf{k}) \quad=$ $\operatorname{diag}\left[\epsilon_{1}(\mathbf{k}), \epsilon_{2}(\mathbf{k}), \ldots, \epsilon_{N}(\mathbf{k})\right]$ the diagonal matrix of energy eigenvalues. Without loss of generality, the chemical potential can be defined to be zero, and the eigenvalues can be arranged in ascending order. For an insulator with $M$ bands filled, one has

$$
\begin{aligned}
\epsilon_{1}(\mathbf{k}) & \leq \epsilon_{2}(\mathbf{k}) \leq \ldots \leq \epsilon_{M}(\mathbf{k})<0 \\
& <\epsilon_{M+1}(\mathbf{k}) \leq \ldots \leq \epsilon_{N}(\mathbf{k}) .
\end{aligned}
$$

For $t \in[0,1]$, define

$$
E_{\alpha}(\mathbf{k}, t)=\left\{\begin{array}{l}
\epsilon_{\alpha}(\mathbf{k})(1-t)+\epsilon_{G} t, \quad 1 \leq \alpha \leq M \\
\epsilon_{\alpha}(\mathbf{k})(1-t)+\epsilon_{E} t, \quad M<\alpha \leq N
\end{array}\right.
$$

and $D_{0}(\mathbf{k}, t)=\operatorname{diag}\left[E_{1}(\mathbf{k}, t), E_{2}(\mathbf{k}, t), \ldots, E_{N}(\mathbf{k}, t)\right]$, then we have

$D_{0}(\mathbf{k}, 0)=D(\mathbf{k}), D_{0}(\mathbf{k}, 1)=\left(\begin{array}{ll}\epsilon_{G} \mathbb{I}_{M \times M} & \\ & \epsilon_{E} \mathbb{I}_{N-M \times N-M}\end{array}\right)$.

As long as $\epsilon_{G}<0<\epsilon_{H}, D_{0}(\mathbf{k}, t)$ remains gapped for $t \in[0,1]$. Thus by defining

$$
h(\mathbf{k}, t)=U(\mathbf{k}) D_{0}(\mathbf{k}, 0) U^{\dagger}(\mathbf{k})
$$

we obtain an adiabatic interpolation between $h(\mathbf{k}, 0)=$ $h(\mathbf{k})$ and $h(\mathbf{k}, 1)=U(\mathbf{k}) D_{0}(\mathbf{k}, 1) U^{\dagger}(\mathbf{k})$. Since the matrix $U(\mathbf{k})$ can be written in the eigenstates of $h(\mathbf{k})$ as $U(\mathbf{k})=$ $(|1, \mathbf{k}\rangle,|2, \mathbf{k}\rangle, \ldots,|N, \mathbf{k}\rangle)$, we have

$$
\begin{aligned}
h(\mathbf{k}, 1) & =\epsilon_{G} \sum_{\alpha=1}^{M}|\alpha, \mathbf{k}\rangle\left\langle\alpha, \mathbf{k}\left|+\epsilon_{E} \sum_{\beta=M+1}^{N}\right| \beta, \mathbf{k}\right\rangle\langle\beta, \mathbf{k}| \\
& =\epsilon_{G} P_{G}(\mathbf{k})+\epsilon_{E} P_{E}(\mathbf{k})
\end{aligned}
$$

In summary, we have proven that each gapped Hamiltonian $h(\mathbf{k})$ can be adiabatically connected to a Hamiltonian with the form of Eq. (55).

\section{Calculation of correlation function (53) for $h_{0}(\mathbf{k})$}

For Hamiltonian of the form (B5) the Green's function is written in the simple form:

$$
\begin{aligned}
G(\mathbf{k}, \omega) & =\left[\omega+i \delta-\epsilon_{G} P_{G}(\mathbf{k})-\epsilon_{E} P_{E}(\mathbf{k})\right]^{-1} \\
& =\frac{P_{G}(\mathbf{k})}{\omega+i \delta-\epsilon_{G}}+\frac{P_{E}(\mathbf{k})}{\omega+i \delta-\epsilon_{E}}
\end{aligned}
$$

On the other hand, we have

$$
\begin{aligned}
\frac{\partial G^{-1}(\mathbf{k}, \omega)}{\partial \omega} & =1 \\
\frac{\partial G^{-1}(\mathbf{k}, \omega)}{\partial k_{i}} & =-\epsilon_{G} \frac{\partial P_{G}(\mathbf{k})}{\partial k_{i}}-\epsilon_{E} \frac{\partial P_{E}(\mathbf{k})}{\partial k_{i}} \\
& =\left(\epsilon_{E}-\epsilon_{G}\right) \frac{\partial P_{G}(\mathbf{k})}{\partial k_{i}}
\end{aligned}
$$

where $i=1,2,3,4$. Thus Eq. (53) can be written

$$
C_{2}=-\frac{\pi^{2}}{3} \epsilon^{i j k \ell} \int \frac{d^{4} k d \omega}{(2 \pi)^{5}} \sum_{n, m, s, t=1,2} \frac{\operatorname{Tr}\left[P_{n} \frac{\partial P_{G}}{\partial k_{i}} P_{m} \frac{\partial P_{G}}{\partial k_{j}} P_{s} \frac{\partial P_{G}}{\partial k_{k}} P_{t} \frac{\partial P_{G}}{\partial k_{\ell}}\right]\left(\epsilon_{E}-\epsilon_{G}\right)^{4}}{\left(\omega+i \delta-\epsilon_{n}\right)^{2}\left(\omega+i \delta-\epsilon_{m}\right)\left(\omega+i \delta-\epsilon_{s}\right)\left(\omega+i \delta-\epsilon_{t}\right)}
$$

in which $\epsilon_{1,2}=\epsilon_{G, E}$ and $P_{1,2}(\mathbf{k})=P_{G, E}(\mathbf{k})$, respectively. From the identity $P_{E}+P_{G} \equiv \mathbb{I}$ and $P_{E}^{2}=P_{E}, P_{G}^{2}=P_{G}$, we obtain

$$
P_{E} \frac{\partial P_{G}}{\partial k_{i}}=-\frac{\partial P_{E}}{\partial k_{i}} P_{G}=\frac{\partial P_{G}}{\partial k_{i}} P_{G}, P_{G} \frac{\partial P_{G}}{\partial k_{i}}=-P_{G} \frac{\partial P_{E}}{\partial k_{i}}=\frac{\partial P_{G}}{\partial k_{i}} P_{E}
$$


Consequently, $P_{G} \partial_{i} P_{G} P_{G}=P_{E} \partial_{i} P_{G} P_{E}=0$, so that the trace in Eq. (B8) can be nonzero only when $n \neq m, m \neq$ $s, s \neq t, t \neq n$. In other words, only two terms are left out of the 16 terms summed over in Eq. (B8):

$$
C_{2}=-\frac{\pi^{2}}{3} \epsilon^{i j k \ell} \int \frac{d^{4} k d \omega}{(2 \pi)^{5}}\left\{\frac{\operatorname{Tr}\left[P_{G} \frac{\partial P_{G}}{\partial k_{i}} P_{E} \frac{\partial P_{G}}{\partial k_{j}} P_{G} \frac{\partial P_{G}}{\partial k_{k}} P_{E} \frac{\partial P_{G}}{\partial k_{\ell}}\right]}{\left(\omega+i \delta-\epsilon_{G}\right)^{3}\left(\omega+i \delta-\epsilon_{E}\right)^{2}}+\frac{\operatorname{Tr}\left[P_{E} \frac{\partial P_{G}}{\partial k_{i}} P_{G} \frac{\partial P_{G}}{\partial k_{j}} P_{E} \frac{\partial P_{G}}{\partial k_{k}} P_{G} \frac{\partial P_{G}}{\partial k_{\ell}}\right]}{\left(\omega+i \delta-\epsilon_{G}\right)^{2}\left(\omega+i \delta-\epsilon_{E}\right)^{3}}\right\}\left(\epsilon_{E}-\epsilon_{G}\right)^{4} .
$$

Carrying out the integral over $\omega$ and using identities (B9) again, we obtain

$$
C_{2}=\frac{1}{8 \pi^{2}} \int d^{4} k \epsilon^{i j k \ell} \operatorname{Tr}\left[P_{E} \frac{\partial P_{G}}{\partial k_{i}} \frac{\partial P_{G}}{\partial k_{j}} P_{E} \frac{\partial P_{G}}{\partial k_{k}} \frac{\partial P_{G}}{\partial k_{\ell}}\right] .
$$

Now we will show this is just the second Chern number. The Berry phase gauge field is defined by

$$
a_{i}^{\alpha \beta}(\mathbf{k})=-i\left\langle\alpha, \mathbf{k}\left|\frac{\partial}{\partial k_{i}}\right| \beta, \mathbf{k}\right\rangle
$$

in which $\alpha, \beta=1,2, . ., M$ are the occupied bands. The $U(M)$ gauge curvature is given by

$$
\begin{aligned}
f_{i j}^{\alpha \beta}= & \partial_{i} a_{j}^{\alpha \beta}-\partial_{j} a_{i}^{\alpha \beta}+i\left[a_{i}, a_{j}\right]^{\alpha \beta} \\
= & -i\left(\frac{\partial\langle\alpha, \mathbf{k}|}{\partial k_{i}} \frac{\partial|\beta, \mathbf{k}\rangle}{\partial k_{j}}-(i \leftrightarrow j)\right) \\
& +i\left(\frac{\partial\langle\alpha, \mathbf{k}|}{\partial k_{i}} \sum_{\gamma=1}^{M}|\gamma, \mathbf{k}\rangle\langle\gamma, \mathbf{k}| \frac{\partial|\beta, \mathbf{k}\rangle}{\partial k_{j}}-(i \leftrightarrow j)\right) \\
= & -i\left(\frac{\partial\langle\alpha, \mathbf{k}|}{\partial k_{i}} P_{E}(\mathbf{k}) \frac{\partial|\beta, \mathbf{k}\rangle}{\partial k_{j}}-(i \leftrightarrow j)\right)
\end{aligned}
$$

in which $(i \leftrightarrow j)$ means the term with $i, j$ exchanged. In operator form, we have

$$
\sum_{\alpha, \beta=1}^{M}|\alpha, \mathbf{k}\rangle f_{i j}^{\alpha \beta}\langle\beta, \mathbf{k}|=-i \frac{\partial P_{G}(\mathbf{k})}{\partial k_{i}} P_{E} \frac{\partial P_{G}(\mathbf{k})}{\partial k_{j}}-(i \leftrightarrow j) .
$$

Thus Eq. (B10) can be written in terms of the Berry phase curvature as ${ }^{42,46}$

$$
C_{2}=\frac{1}{32 \pi^{2}} \int d^{4} k \epsilon^{i j k \ell} \operatorname{Tr}\left[f_{i j} f_{k \ell}\right] .
$$

In conclusion, the equivalence of the non-linear correlation function (53) and the second Chern number (54) has been proven for the specific models of the form Eq. (55). Due to the topological invariance of both Eq. (53) and the second Chern number, such a relation holds for any band insulator in $(4+1)$-d.

The procedure in this section can be easily generalized to any odd space-time dimensions. In $(2 n+1)$-d spacetime, a $n$-th Chern number $C_{n}$ is defined in the BZ of a band-insulator, which appears as a topological response coefficient to an external gauge field. Equivalently, the coefficient of the $n$-th Chern-Simons term in the effective action of an external gauge field obtained from integrating out the fermions 40 .

\section{APPENDIX C: THE WINDING NUMBER IN THE NON-LINEAR RESPONSE OF DIRAC-TYPE MODELS}

Starting from the symmetric form, in terms of general Green's functions, of Eq. (53) we want to calculate $C_{2}$ for the generalized lattice Dirac model $H(k)=d_{a}(k) \Gamma^{a}$. The Green's function for this model can be simplified to $\underline{40}$

$$
G(k, \omega)=\frac{\omega+d^{a}(k) \Gamma^{a}}{\left(\omega^{2}-d^{a}(k) d_{a}(k)\right)}
$$

Inserting this into Eq. (53) yields the following calculation

$$
\begin{aligned}
C_{2} & =-\frac{\pi^{2}}{3} \epsilon_{i j k l} \int \frac{d^{4} k d \omega}{(2 \pi)^{5}} \frac{1}{\left(\omega^{2}-|d|^{2}\right)^{5}} \operatorname{Tr}\left[\left(\omega+d_{m} \Gamma^{m}\right)\left(\omega+d_{n} \Gamma^{n}\right)\left(\partial_{i} d^{o} \Gamma^{o}\right)\left(\omega+d_{p} \Gamma^{p}\right)\left(\partial_{j} d^{q} \Gamma^{q}\right)\right. \\
& \left.\times\left(\omega+d_{r} \Gamma^{r}\right)\left(\partial_{k} d^{s} \Gamma^{s}\right)\left(\omega+d_{t} \Gamma^{t}\right)\left(\partial_{l} d^{u} \Gamma^{u}\right)\right] \\
& =-\frac{\pi^{2}}{3} \epsilon_{i j k l} \int \frac{d^{4} k d \omega}{(2 \pi)^{5}} \frac{\partial_{i} d^{o} \partial_{j} d^{q} \partial_{k} d^{s} \partial_{l} d^{u}}{\left(\omega^{2}-|d|^{2}\right)^{5}} \operatorname{Tr}\left[\left(\omega+d_{m} \Gamma^{m}\right)\left(\omega+d_{n} \Gamma^{n}\right) \Gamma^{o}\left(\omega+d_{p} \Gamma^{p}\right) \Gamma^{q}\left(\omega+d_{r} \Gamma^{r}\right) \Gamma^{s}\left(\omega+d_{t} \Gamma^{t}\right) \Gamma^{u}\right]
\end{aligned}
$$

where $m, n, o, p, q, r, s, t, u=0,1,2,3,4$. Among all the terms in the bracket [], the only ones with non-zero traces are those of 5,7 , and $9 \Gamma$ matrices 42 and the complete trace simplifies nicely to $-4 \epsilon_{\text {toqsu }} d^{t}\left(\omega^{2}-|d|^{2}\right)^{2}$, which 
simplifies $C_{2}$ as

$$
\begin{aligned}
C_{2} & =\frac{\pi^{2}}{3} \epsilon_{i j k l} \int \frac{d^{4} k d \omega}{(2 \pi)^{5}} 4 \epsilon_{\text {toqsu }} \frac{d^{t} \partial_{i} d^{o} \partial_{j} d^{q} \partial_{k} d^{s} \partial_{l} d^{u}}{\left(\omega^{2}-|d|^{2}\right)^{3}} \\
& =\frac{\pi^{2}}{4} \epsilon_{i j k l} \int \frac{d^{4} k}{(2 \pi)^{4}} \epsilon_{\text {toqsu }} \frac{d^{t} \partial_{i} d^{o} \partial_{j} d^{q} \partial_{k} d^{s} \partial_{l} d^{u}}{|d|^{5}} \\
& =\frac{3}{8 \pi^{2}} \int d^{4} k \epsilon_{\text {toqsu }} \hat{d}^{t} \partial_{x} \hat{d}^{o} \partial_{y} \hat{d}^{q} \partial_{z} \hat{d}^{s} \partial_{w} \hat{d}^{u} .
\end{aligned}
$$

Thus we have proved that the winding number given in the expression (64) is equal to the second Chern number defined in Eq. (53) for generic $(4+1)$-d Dirac models.

\section{APPENDIX D: STABILITY OF EDGE THEORIES IN GENERIC DIMENSIONS}

In this appendix, we will determine the existence or absence of $Z_{2}$ topological insulators in generic dimensions by studying the stability of boundary theories. First of all, the boundary theory of a topological insulator in $(2 n+1)$-d with nontrivial $n$-th Chern number $C_{n}$ is $\left|C_{n}\right|$ copies of chiral fermions:

$$
H=\operatorname{sgn}\left(C_{n}\right) \sum_{s=1}^{\left|C_{n}\right|} \sum_{a=1}^{2 n-1} \psi_{s}^{\dagger} v p_{a} \Gamma^{a} \psi_{s}
$$

The $2 n-1 \Gamma^{a}$ matrices are $2^{n-1}$ dimensional and form an so $(2 n-1)$ Clifford algebra. We will study the lattice Dirac model, since other systems with the same Chern number can be obtained by an adiabatic deformation from the lattice Dirac model and the topological stability of edge states does not depend on the adiabatic deformation, as will be discussed below. For simplicity, in the following we will focus on the case $C_{n}=1$, in which case the single particle Hamiltonian can be written as $h(\mathbf{p})=v p_{a} \Gamma^{a}$. We have the following theorem about the symmetry of the Hamiltonian:

- Theorem I. In a $2^{n-1}$ dimensional representation of an so $(2 \mathrm{n}-1)$ Clifford algebra generated by $\Gamma^{a}, a=1,2, . .2 n-1$, a unitary matrix $M_{(n)}$ can be defined such that

$$
\begin{aligned}
M_{(n)}^{\dagger} \Gamma^{a} M_{(n)} & =(-1)^{n-1} \Gamma^{a *}, \forall a=1,2, . ., 2 n-1 \\
M_{(n)}^{*} M_{(n)} & =(-1)^{[n / 2]} \mathbb{I}
\end{aligned}
$$

Consequently the chiral Hamiltonian $h(\mathbf{p})=v p_{a} \Gamma^{a}$ satisfies

$$
M_{(n)}^{\dagger} h(\mathbf{p}) M_{(n)}=(-1)^{n} h^{*}(-\mathbf{p}) .
$$

In Eq. (D3) $[n / 2]$ means the maximal integer that does not exceed $n / 2$.

Theorem I can be proved by induction. First, the $\Gamma^{a}$ matrices for $n=2$ are the Pauli matrices $\Gamma^{a}=\sigma_{a}, a=$
$1,2,3$, and $M_{(2)}=i \sigma_{2}$ satisfies the theorem. Suppose the theorem is true for case $n$ with the matrix $M_{(n)}$ and the $2 n-1$ Gamma matrices $\Gamma_{(2 n-1)}^{a}$, then the $\Gamma_{(2 n+1)}^{a}$ matrices for the $\operatorname{so}(2 n+1)$ Clifford algebra can be generated by

$$
\Gamma_{(2 n+1)}^{a}=\left\{\begin{array}{cc}
\Gamma_{(2 n-1)}^{a} \otimes \tau_{y}, & a=1,2, . .2 n-1 \\
\mathbb{I} \otimes \tau_{x}, & a=2 n \\
\mathbb{I} \otimes \tau_{z}, & a=2 n+1
\end{array} .\right.
$$

It is straightforward to check the anticommutation relations. Defining

$$
M_{(n+1)}=\left\{\begin{array}{cc}
M_{(n)} \otimes i \tau_{y}, & n \text { odd } \\
M_{(n)} \otimes \mathbb{I}, & n \text { even }
\end{array}\right.
$$

we find $M_{(n+1)}$ satisfies the Theorem I.

From Eqs. (D3) and (D4) one finds different properties of $M_{(n)}$ in different dimensions. (i) For $n=$ $4 k-3, k \in \mathbb{Z}, M_{(n)}$ reverses the sign of energy, and satisfies $M_{(n)}^{*} M_{(n)}=\mathbb{I}$, which can be identified as particlehole symmetry; (ii) for $n=4 k-2, k \in \mathbb{Z}, M_{(n)}$ preserves the energy and satisfies $M_{(n)}^{*} M_{(n)}=-\mathbb{I}$, which can be identified as the time-reversal symmetry; (iii) for $n=4 k-1, k \in \mathbb{Z}, M_{(n)}$ reverses the sign of energy but satisfies $M_{(n)}^{*} M_{(n)}=-\mathbb{I}$, which we call "psuedo" particlehole symmetry and denote by $\tilde{C}$; (iv) for $n=4 k, k \in \mathbb{Z}$, $M_{(n)}$ preserves the energy but satisfies $M_{(n)}^{*} M_{(n)}=\mathbb{I}$, which behaves like the time-reversal symmetry of an integer-spin particle, and we call "pseudo" time-reversal symmetry $\tilde{T}$.

In the following, we will denote the symmetries $C, \tilde{C}$, $T$ and $\tilde{T}$, defined by Eq. (D4), by $M$-symmetry. Now we study what other terms can be added in the Hamiltonian without breaking the $M$ symmetry. Given $\Gamma^{a}$ for the so $(2 \mathrm{n}-1)$ case, all the $2^{n-1} \times 2^{n-1}$ Hermitian matrices can be expanded in the basis

$$
\left\{\mathbb{I}, \Gamma^{a_{1} a_{2} \ldots a_{m}}=i^{m(m-1) / 2} \Gamma^{a_{1}} \Gamma^{a_{2}} \ldots \Gamma^{a_{m}}, m=1,2, . . n-1\right\} .
$$

As expected, the total number of matrices forming the basis is $1+\sum_{m=1}^{n-1}\left(\begin{array}{c}m \\ 2 n-1\end{array}\right)=2^{2 n-2}$. The $M_{(n)}$ transformation property of $\Gamma^{a_{1} a_{2} . . a_{m}}$ can be determined by that of $\Gamma^{a}$ as

$$
M_{(n)}^{\dagger} \Gamma_{(n)}^{a_{1} a_{2} . . a_{m}} M_{(n)}=(-1)^{m(2 n+m-3) / 2} \Gamma_{(n)}^{a_{1} a_{2} . . a_{m} *}
$$

If we have a constant term $m_{a_{1} a_{2} . . a_{m}} \Gamma^{a_{1} a_{2} . . a_{m}}$ in the Hamiltonian without breaking the $M$ symmetry, the following condition must be satisfied:

$$
M^{\dagger} \Gamma^{a_{1} a_{2} . . a_{m}} M=(-1)^{n} \Gamma^{a_{1} a_{2} . . a_{m} *} .
$$

Eqs. (D7) and (D8) thus require

$$
(-1)^{m(2 n+m-3) / 2}=(-1)^{n}
$$

so that $(m-1)(2 n-2+m) / 2$ must be odd. This condition can be satisfied by several possibilities: (1) when $m$ is 
odd, $(m-1) / 2$ must be odd, which means $m=4 k-1, k \in$ $\mathbb{N}$. (2) When $m$ is even, $n-1+m / 2$ must be odd, which means $m=4 k$ if $n$ is even, or $m=4 k-2$ if $n$ is odd. In summary, the terms available in the Hamiltonian are given by

$$
m=\left\{\begin{array}{cc}
4 k-1 \text { or } 4 k, & n \text { even } \\
4 k-1 \text { or } 4 k-2, & n \text { odd }
\end{array},\right.
$$

in which $k \in \mathbb{N}$ and $m$ is also bounded by $1 \leq m<n$. For example, the first non-trivial case is $n=2$ in which $\Gamma^{a}, a=1,2,3$ are Pauli matrices. Since $n$ is even, $m$ is required to be $4 k-1$ or $4 k$, in which the lowest value is $m=3$. Consequently there is no mass term available. When $n=3(2 n-1=5)$ the $\Gamma^{a}, a=1,2, . ., 5$ matrices are the usual Dirac matrices, and the only value of $m$ satisfying Eq. (D10) is $m=2$. In other words, the terms $\Gamma^{a b}=i \Gamma^{a} \Gamma^{b}$ do not break the corresponding symmetrythe pseudo particle-hole symmetry $\tilde{C}$.

Though there are all these constant terms available, the perturbed Hamiltonian $h(k)=k_{a} \Gamma^{a}+$ $\sum_{m} m_{a_{1} a_{2} . . a_{m}} \Gamma^{a_{1} a_{2} . . a_{m}}$ remains gapless because each $\Gamma^{a_{1} a_{2} . . a_{m}}$ commutes with some $\Gamma^{a}$. If $m$ is even, it commutes with $\Gamma^{b}, b \neq a_{s} \forall s=1, . ., m$. If $m$ is odd, it commutes with $\Gamma^{a_{s}}, \forall s=1, . ., m$. For the Hamiltonian with only one constant term $m_{a_{1} a_{2} \ldots a_{m}} \Gamma^{a_{1} a_{2} \ldots a_{m}}$, one can take $p_{i}=0$ for all $i$ except for $i=a$, where $a$ is chosen such that $\Gamma^{a}$ commutes with $\Gamma^{a_{1} a_{2} . . a_{m}}$. Due to the commutativity, the Hamiltonian can be diagonalized along the $a$-th axis, with the eigenvalues $p_{a} \pm m$. Consequently, we know the Hamiltonian is gapless. In other words, we have shown that no perturbation with the $2^{n-1}$ band theory can open a gap for the $2 n$ dimensional chiral fermion, which agrees with the topological stability of the $(2 n+1)$ $\mathrm{d}$ bulk system characterized by the $n$-th Chern number.

Starting from the $(2 n+1)$-d topological insulator, dimensional reduction procedures can be carried out to obtain a $((2 n-1)+1)$-d topological insulator. Correspondingly, the edge theory of the $((2 n-2)+1)-d$ topological insulator is given by the dimensional reduction of the $((2 n-1)+1)$-d chiral fermion. If a nontrivial topological insulator can be defined, it has the boundary theory $h(\mathbf{p})=\sum_{a=1}^{2 n-2} p_{a} \Gamma^{a}$. Compared to the $((2 n-1)+1)-d$ chiral fermion, one $\Gamma^{a}$ matrix is absent in the theory. In the same way, the boundary theory of lower dimensional descendants can be obtained by removing more momenta and $\Gamma^{a}$ matrices from the chiral fermion Hamiltonian. Obviously, if too few $\Gamma^{a}$ matrices are left, a mass term will be available, which anticommutes with all the rest of the $\Gamma^{a}$ matrices and thus can make gap the whole edge spectrum. The upper critical dimension where the edge states become unstable is determined by the maximal number of $\Gamma^{a}$ matrices that anti-commute with some $\Gamma^{a_{1} a_{2} . . a_{m}}$. For $m$ even, the $\max$ imal number of $\Gamma^{a}$ 's that anticommute with $\Gamma^{a_{1} a_{2} . . a_{m}}$ is $m$, while for $m$ odd, the maximal number is $2 n-1-m$. On the other hand, the available values of $m$ are defined by the constraint Eq. (D10). By studying the cases $n=4 k-3,4 k-2,4 k-1,4 k, k \in \mathbb{N}$ separately, we obtain that the space-time dimension in which the chiral theory becomes unstable is given by $d=2 n-3$. For example, in the case $n=3$ discussed earlier, the mass terms $\Gamma^{a b}$ are permitted by the pseudo particle-hole symmetry. When the dimension is reduced from $(5+1)$-d to $(2+1)$-d, only two $\Gamma^{a}$ matrices are used in the $k$-linear terms, so that some $\Gamma^{a b}$ can be found which anti-commutes with the gapless Hamiltonian and thus can make the surface theory gapped. Consequently, topologically stable boundary theories as descendants of $((2 n-1)+1)$-d chiral fermions can only exist in $((2 n-2)+1)$ and $((2 n-3)+1)$ dimensions. Correspondingly, the $Z_{2}$ topological insulators as descendants of the $(2 n+1)$-d topological insulator (with $n$-th Chern number) can only be defined in $((2 n-1)+1)$ and $((2 n-2)+1)$ dimensions.
${ }^{1}$ K. v. Klitzing, G. Dorda, and M. Pepper, Phys. Rev. Lett. 45, 494 (1980).

2 D. C. Tsui, H. L. Stormer, and A. C. Gossard, Phys. Rev. Lett. 48, 1559 (1982).

3 R. B. Laughlin, Phys. Rev. B 23, 5632 (1981).

4 R. B. Laughlin, Phys. Rev. Lett. 50, 1395 (1983).

5 D. J. Thouless, M. Kohmoto, M. P. Nightingale, and M. den Nijs, Phys. Rev. Lett. 49, 405 (1982).

${ }^{6}$ Q. Niu, D. J. Thouless, and Y.-S. Wu, Phys. Rev. B 31, 3372 (1985).

7 S. C. Zhang, T. H. Hansson, and S. Kivelson, Phys. Rev. Lett. 62, 82 (1989).

8 S. C. Zhang, Int. J. Mod. Phys. B 6, 25 (1992).

9 W. P. Su, J. R. Schrieffer, and A. J. Heeger, Phys. Rev. Lett. 42, 1698 (1979).

10 R. D. King-Smith and D. Vanderbilt, Phys. Rev. B 47, 1651 (1993).

11 G. Ortiz and R. M. Martin, Phys. Rev. B 49, 14202 (1994).

12 D. J. Thouless, Phys. Rev. B 27, 6083 (1983).
13 J. Goldstone and F. Wilczek, Phys. Rev. Lett. 47, 986 (1981).

14 S. C. Zhang and J. P. Hu, Science 294, 823 (2001).

15 B. A. Bernevig, C. H. Chern, J. P. Hu, N. Toumbas, and S. C. Zhang, Annals of Physics 300, 185 (2002).

16 C. L. Kane and E. J. Mele, Phys. Rev. Lett. 95, 226801 (2005).

17 B.A. Bernevig and S.C. Zhang, Phys. Rev. Lett. 96, 106802 (2006).

18 C. Wu, B.A. Bernevig, and S.C. Zhang, Phys. Rev. Lett. 96, 106401 (2006).

19 C. Xu and J. Moore, Phys. Rev. B 73, 045322 (2006).

20 B. A. Bernevig, T. L. Hughes, and S.C. Zhang, Science 314, 1757 (2006).

21 M. König, S. Wiedmann, C. Brüne, A. Roth, H. Buhmann, L. Molenkamp, X.-L. Qi, and S.-C. Zhang, Science 318, 766 (2007).

${ }^{22}$ L. Fu, C. L. Kane, and E. J. Mele, Phys. Rev. Lett. 98, 106803 (2007). 
23 J. E. Moore and L. Balents, Phys. Rev. B 75, 121306 (2007).

24 R. Roy, arxiv: cond-mat/0604211.

25 S. Murakami, N. Nagaosa, and S.C. Zhang, Phys. Rev. Lett. 93, 156804 (2004).

26 C. L. Kane and E. J. Mele, Phys. Rev. Lett. 95, 146802 (2005).

27 L. Fu and C. L. Kane, Phys. Rev. B 74, 195312 (2006).

28 L. Fu and C. L. Kane, Phys. Rev. B 76, 045302 (2007).

29 R. Roy, arxiv: cond-mat/0607531

30 R. Roy, arxiv: cond-mat/0608064.

31 X. L. Qi and S. C. Zhang, arxiv: cond-mat/0801.0252.

${ }^{32}$ Y. Ran, A. Vishwanath, and D.-H. Lee, arxiv: condmat/0801.0627.

33 A. J. Niemi and G. W. Semenoff, Phys. Rev. Lett. 51, 2077 (1983).

34 J. von Neumann and E. Wigner, Phys. Z. 30, 467 (1929).

${ }^{35}$ X.L. Qi, Y.S. Wu, and S.C. Zhang, Phys. Rev. B 74, 045125 (2006).

${ }^{36}$ G. E. Volovik, The Universe in a Helium Droplet (Oxford University Press,USA, 2003).

37 F. D. M. Haldane, Phys. Rev. Lett. 61, 2015 (1988).

${ }^{38}$ Chaoxing Liu, Xiaoliang Qi, Xi Dai, Zhong Fang and Shoucheng Zhang, arXiv:0802.2711

39 R. Jackiw and C. Rebbi, Phys. Rev. D 13, 3398 (1976).

40 M. F. L. Golterman, K. Jansen, and D. B. Kaplan, Phys. Lett. B 301, 219 (1993).

41 G. E. Volovik, JETP Lett. 75, 63 (2002).

42 S. Murakami, N. Nagaosa, and S.C. Zhang, Phys. Rev. B 69, 235206 (2004).

43 M. Creutz, Rev. Mod. Phys. 73, 119 (2001).

44 We use the capital $\Gamma^{a}$ here since they are defined differently from the usual Dirac matrix $\gamma^{a}, a=0,1,2,3,5$. The relation between $\Gamma^{a}$ and $\gamma^{a}$ is $\Gamma^{0}=\gamma^{0}, \Gamma^{1,2,3,4}=-i \gamma^{0} \gamma^{1,2,3,5}$.

45 J. E. Avron, L. Sadun, J. Segert, and B. Simon, Phys. Rev. Lett. 61, 1329 (1988).

46 E. Demler and S. C. Zhang, Annals of Physics 271, 83 (1999).

47 S. Adler, Phys. Rev. 177, 2426 (1969).

48 J. S. Bell and R. Jackiw, Nuovo Cimento A 60, 47 (1969).

49 D. Xiao, J. Shi, D. P. Clougherty, and Q. Niu, arxiv: condmat/0711.1855.

50 M. C. Huang and P. Sikivie, Phys. Rev. D 32, 1560 (1985).

${ }^{51}$ F. Wilczek, Phys. Rev. Lett. 58, 1799 (1987).
${ }^{52}$ K. Lee, Phys. Rev. D 35, 3286 (1987).

53 R. D. Peccei and H. R. Quinn, Phys. Rev. Lett. 38, 1440 (1977).

54 P. Sikivie, Phys. Lett. B 137, 353 (1984).

${ }^{55}$ C. G. Callan, R. F. Dashen, and D. J. Gross, Phys. Lett. B 63, 334 (1976).

${ }^{56}$ R. Jackiw and C. Rebbi, Phys. Rev. Lett. 37, 172 (1976).

57 X. Dai and et al., arxiv: 0705.1516.

58 E. Fradkin, E. Dagotto, and D. Boyanovsky, Phys. Rev. Lett. 57, 2967 (1986).

59 N. Hur, S. Park, P. A. Sharma, J. S. Ahn, S. Guha, and S.-W. Cheong, Nature 429, 392 (2004).

60 W. Eerenstein, N. D. Mathur, and J. F. Scott, Nature 442, 759 (2006).

61 E. Witten, Phys. Lett. B 86, 283 (1979).

62 More explicitly, if we choose the representation $\Gamma^{0}=\sigma^{3} \otimes \mathbb{I}$, $\Gamma^{1,2,3}=\sigma^{1} \otimes \tau^{1,2,3}$ and $\Gamma^{4}=\sigma^{2} \otimes \mathbb{I}$, then the time-reversal transformation matrix $T$ is defined as $T=\mathbb{I} \otimes i \tau^{2}$.

63 B. Halperin, Japanese Journal of Applied Physics Suppl. 26, 1913 (1987).

64 A. N. Redlich, Phys. Rev. D 29, 2366 (1984).

65 T. Jaroszewicz, Physics Letters B 146, 337 (1984).

66 A. G. Abanov and P. B. Wiegmann, Journal of High Energy Physics 10, 30 (2001).

67 C. Chamon, C.-Y. Hou, R. Jackiw, C. Mudry, S.-Y. Pi, and A. P. Schnyder, Arxiv preprint 0707.0293 (2007).

68 T. Grover and T. Senthil, Arxiv preprint 0801.2130 (2008).

69 X.-L. Qi, T. L. Hughes, and S.-C. Zhang, e-print arXiv:0710.0730

70 B. Julia and A. Zee, Phys. Rev. D 11, 2227 (1975).

71 M. Nakahara, Geometry, Topology, and Physics (A. Hilger, 1990).

72 D. B. Kaplan, Phys. Lett. B 288, 342 (1992).

73 T. Kaluza, Sitzungsber. Preuss. Akad. Wiss. Berlin. (Math. Phys.) p. 966 (1921).

74 O. Klein, Z. Phys. 37, 895 (1926).

75 H. B. Lawson and M.-L. Michelsohn, Spin Geometry (Princeton Press,Princeton,NJ, 1989).

76 N. Read and D. Green, Phys. Rev. B 61, 10267 (2000).

77 D. A. Ivanov, Phys. Rev. Lett. 86, 268 (2001).

78 M. Stone and R. Roy, Phys. Rev. B 69, 184511 (2004).

79 C. G. Callan and J. A. Harvey, Nucl. Phys. B 250, 427 (1985). 